Electronic Excited States as a Probe of Surface Adsorbate structure and Dynamics in Liquid Xenon

LBL -3284 ?

by

DE93 002550

Eric Scott Peterson

Ph.D. Thesis

Department of Chemistry

University of California

and

Chemical Sciences Division

Lawrence Berkeley Laboratory

University of California

Berkeley, CA 94720

August, 1992

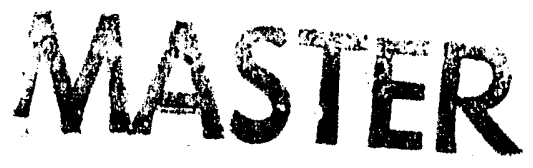

This work was supported by the Director, office of Energy Research, Office of Basic Energy Sciences, Chemical Sciences Division of the U. S. Department of Energy, under Contract No. DE-AC03-76SF00098. 


\title{
Electronic Excited States as a Probe of Surface Adsorbate Structure and Dynamics in Liquid Xenon
}

Copyright $\odot 1992$

by

\author{
Eric Scott Peterson
}

The U.S. Department of Energy has the right to use this thesis for any purpose whatsoever including the right to reproduce all cr any part thereof 


\title{
Electronic Excited states as a Probe of Surface Adsorbate Structure and Dynamics in Liquid Xenon
}

by

Eric scott Peterson

\begin{abstract}
A combination of second harmonic generation (SHG) and a simple dipole-dipole interaction model is presented as a new technique for determining adsorbate geometries on surfaces. The polarization dependence of SHG is used to define possible geometries of the adsorbate about the surface normal. General formulae for absorption band shifts using the geometry constraints imposed by the SHG data are derived for a dimer constructed from two arbitrarily placed monomers on the surface using the dipole-dipole interaction potential. These formulae can be used to determine the orientation of the two monomers relative to each other. A simplified version of this formalism is used to interpret absorption band shifts for rhodamine B adsorbed on fused silica.

A brief history of the development of the exciton is given with particular detail to points relevant to excitons in xenon. Data are presented for transient absorption experiments conducted at room temperature in liquid xenon on the picosecond time scale. To my knowledge, these are the first direct observations in liquid xenon at room temperature of
\end{abstract}


both tunneling through the barrier that separates the free and trapped exciton states and the subsequent trapping of the exciton. In high densities both of these processes are found to occur within 2 to 6 picoseconds in agreement with the theories of Kmiecik and Schreiber and of Martin. A threshold density is observed that separates relaxation via single binary collisions and relaxation that proceeds via Martin's resonant energy transfer hopping mechanism. Proposals are made for future work on this system. 
To Mom and Dad 


\section{Table of Contents}

Abstract . . . . . . . . . . . . . . . . . . 1

Table of contents . . . . . . . . . . . . . . . iii

List of Figures . . . . . . . . . . . . . . . . . . vi

List of Tables . . . . . . . . . . . . . . . viii

Introduction . . . . . . . . . . . . . . . . . . . ix

Acknowledgements . . . . . . . . . . . . . . xvii

\section{Chapter 1}

Determination of Adsorbate Geometries on Surfaces Using a New Combination of Second Harmonic Generation and Absorption Band Shifts . . . . . . . . . . . . . . . . . . 1

1.1 Introduction ................ 1

1.2 Theory for Analysis of Second Harmonic

Generation Data and Absorption Band Shifts . 2

1.3 Example Calculation of Rhodamine B Geometry

on a Fused Silica Surface and Discussion . .

Chapter 2

A Brief History of Excitons and Their Existence in Xenon 34

2.1 Introduction... . . . . . . . . . 34

2.2 Early Experiments Confirm the Existence of Excitons . . . . . . . . . . . 38

2.3 Excitons in Liquids: Do They Exist? . . . 42

2.4 Exciton Formation: Energetics and Dynamics 49 
2.5 Time Resolved Measurements . . . . . . 65 2.5.1 Luminescence Experiments . . . . 65 2.5.2 Transient absorption experiments . . 75

Chapter 3

Experimental Details . . . . . . . . . . . 80

3.1 Laser system And optics common to Transient Absorption and Fluorescence Experiments . . 80

3.2 Transient Absorption Experiments . . . . 83

3.3 Fluorescence Experiments . . . . . . . 88

3.4 High Pressure Cell . . . . . . . . . 89

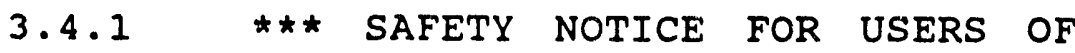
PRESSURE CELL *** . . . . 90

3.4.2 Filling the system with Xenon . . 96

3.4.3 Filling the optical cell with Xenon 98

3.4.4 Trapping the Xenon out of the optical Cell. . . . . . . . . . 100

3.4.5 Final Notes on Cell Construction. 101

Chapter 4

Experimental Data on Relaxation

of Excitons in Condensed Xenon . . . . . . . . . . 104

4.1 Transient Absorption Data . . . . . . 104

4.1 .2 Xenon at $1.8 \mathrm{~g} / \mathrm{ml}$ (810 $\mathrm{psi}$ and

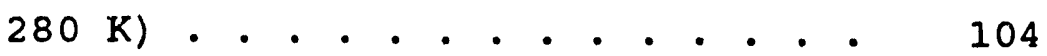

4.1.2 Pressure and Temperature Dependence 112 


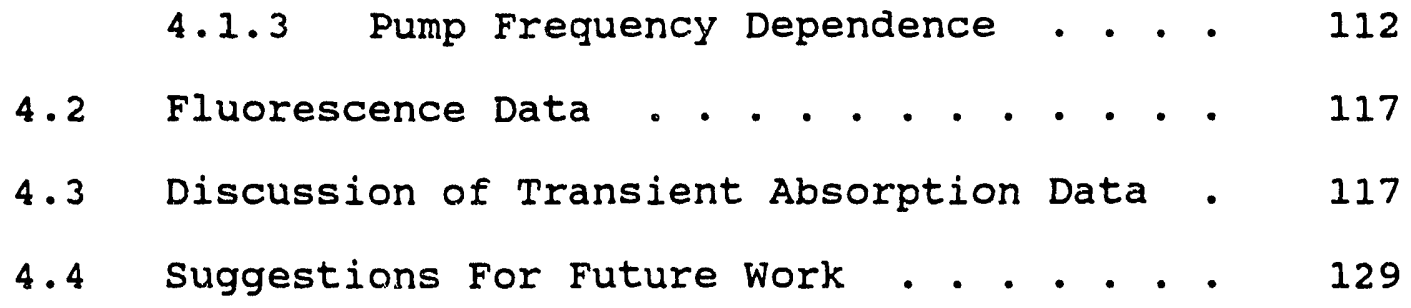

Appendix 1

A Note on the Effects of Impurities in the Xenon . - 133

Appendix 2

Data Collection Subroutines . . . . . . . . . . 137

Subroutine adc.c . . . . . . . . . . . . 137

Subroutine adcshut.c . . . . . . . . . . 141

Subroutine eric.c . . . . . . . . . . . 145

References . . . . . . . . . . . . . . . . . . 147 


\section{List of Fiqures}

Figure 1: Xe atom, exciton, and excimer energy diagram . . . . . . . . . . . . xiv

Figure 2: Coordinates for SHG and dipole equations . 9

Figure 3: Rhodamine $B$ and associated coordinate system . . . . . . . . . . . . 16

Figure 4: Absorption red shift for Rhodamine $B$ on silica . . . . . . . . . . . .

Figure 5: Coordinate system for dipole-dipole interaction . . . . . . . . . . . . 24

Figure 6: Dimer geometries for Rhodamine B on silica 27

Figure 7: synchronously pumped ps dye laser and amplifier . . . . . . . . . . . . 82

Figure 8: Optical setup for transient absorption experiments . . . . . . . . . . . 85

Figure 9: High pressure cell components . . . . . . . 92

Figure 10: Internal details of high pressure optical cell . . . . . . . . . . . . . 94

Figure 11: Transient absorption data for Xe at

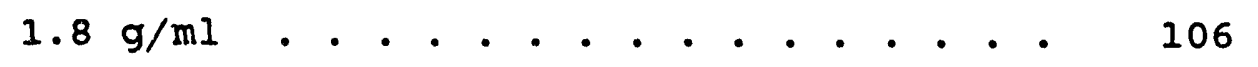

Figure 12: Transient absorption data for Xe at $1.8 \mathrm{~g} / \mathrm{ml}$. . . . . . . . . . . .

Figure 13: Plot of $1.8 \mathrm{~g} / \mathrm{ml}$ fit parameters versus wavelength . . . . . . . . . . 110

Figure 14: Density dependence of Xe dynamics at 
$500 \mathrm{~nm}$. . . . . . . . . . . .

Figure 15: Density dependence of Xe dynamics at $900 \mathrm{~nm}$. . . . . . . . . . . .

Figure 16: Transient absorption processes and Xe dynamics . . . . . . . . . . . 120

Figure 17: Mechanism of exciton trapping proposed by Martin . . . . . . . . . . 128

Figure 18: Effect of oil impurities on long decay . 136 


\section{List of Tables}

Table I: $\quad$ Red Shifts for Rhodamine Dimer Geometries . 28

Table II: Fit parameters for exciton dynamics at

$1.8 \mathrm{~g} / \mathrm{ml}$. . . . . . . . . . . . . . 108

Table III: Fit parameters for exciton density

dependence . . . . . . . . . . 116

viii 


\section{Introduction}

The unifying theme of my research has been the investigation of electronic state interactions, energy transfer, and relaxation processes in the condensed phase with time resolved and continuous wave optical techniques. The proximity of the atoms or molecules to each other in the condensed phase results in perturbations of the gas phase electronic structure. These changes manifest themselves in the formation of energy bands, shifts in transitiun energies, and in changes in the oscillator strengths of transitions. Energy transfer is an important component in a wide range of surface chemistry and dynamics, playing a role from catalysis to solar energy conversion, as well as in the liquid phase where branching ratios and kinetics are controlled by the energetics of reactions. This thesis is divided into two sections, one covering experiments on molecular overlayers on surfaces and energy transfer therein and the other covering relaxation processes of excitons in liquid xenon following excitation with ultraviolet photons.

The motivation for the molecular overlayer studies stemmed from early experiments underway in the Harris lab that focussed on energy transfer from excited molecules in thin molecular overlayers on a surface to the surface and to ground state molecules within the film. The system under study was thin films of pyrene on silicon surfaces, a model for testing 
existing energy transfer theories. The samples were prepared under ultra high vacuum conditions, and the fluorescence was initiated with picosecond pulses from a high repetition rate cavity dumped dye laser that was synchronously pumped with a modelocked argon ion laser. Fluorescence decays were collected using a time correlated single photon counting technique. The decays were found to be markedly non-exponential, suggesting that more than one channel was available for the transfer of energy from the excited pyrene. It was proposed that these cther channels were transfer of energy to trap sites within the film, perhaps dimers or higher order aggregates. 1 These studies did not address the question of how these molecules were arranged within the films to give rise to the trapping effects proposed.

In an effort to understand the intrafilm dynamics by isolating the energy transfer within the film itself from energy transfer to the surface, I conducted experiments on systems of molecular overlayers on insulator surfaces. Fluorescence spectra were collected from tetracene layers on fused sapphire and sodium chloride surfaces in ultra high vacuum using the same techniques as in the pyrene experiments. The peak in the fluorescence spectrum of the tetracene was found to shift to lower energies with increasing surface coverage, suggesting that aggregates were forming on the surface in such a way as to result in the formation of an exciton excited state lowered in energy via dipole-dipole 
coupling with the neighboring ground state molecules.

To address the issue of how molecules in overlayers were arranged, and in particular whether they existed as dimers or higher order aggregates, monolayers of rhodamine $B$ on a quartz surface were studied in air. By combining second harmonic generation (SHG) data and steady state absorption spectra, I was able to obtain information about the distribution of geometries of the molecules on the surface. ${ }^{2}$ second harmonic intensity was measured by Shen et al.$^{3}$ using $P$ and $S$ polarized excitation. The ratio of these two intensities in conjunction with some simple assumptions about the surface distirdbution were used to determine the position of the transition dipole moment of the dye molecules with respect to the surface normal. I measured the absorption spectrum of the dye overlayer as a function of coverage. Shifts in the measured peak position were interpreted with a dipole-dipole interaction model using the dipole orientations from the SHG analysis. From this I was able to determine the orientations of the molecules about these dipole moments and with respect to one another. The most likely configuration for rhodamine $B$ on the surface is an overlapping dimer in which the molecules' transition dipoles are oriented 52 degrees to the surface normal. ${ }^{2}$

The second half of this thesis records experimental studies of relaxation and energy transfer in the liquid phase. Understanding these processes in general is integral to 
understanding phenomena such as reaction kinetics and branching ratios, electron and proton transfer, and intramolecular motions. This particular work is a study of the relaxation dynamics of excimers and excitons created in supercritical and liquid xenon following excitation with a high intensity oV laser beam. This is also of practical importance as condensed xenon has been considered for use as an amplifying medium for vUV lasers."

Picosecond pump prohe transient absorption experiments and fluorescence experiments were conducted to elucidate what rolt the excimers, excitons, and possibly electrons, play in the relaxation of the liquid xenon. In these experiments, the excitation of the xenon is accomplished with a to photon resonantly enhanced absorption, two photons being resonant with a $5 p$ to $6 s$ atomic transition (see Figure 1). It is conceivable that both two and three photon absorptions are possible with our laser intensities, which would create a population of ions, electrons, and excited atoms in the initial step of the experiment. Further experiments will be necessary to elucidate whether or not ionization is occurring. It is well known that an excited xenon atom can combine with a ground state xenon atom in a collision to form an excited dimer, or excimer. In the condensed phase, the excitation produces a free exciton, which then via interaction with the bulk becomes trapped in a state much like that of the gas phase excimer. Much work has been done on the nanosecond time 
Figure 1: Energy level diagram for the xenon atom and free and trapped excitons. The atomic $6 \mathrm{~s}$ manifold correlates to the excimer dimer states at infinite internuclear separation in the gas phase. In the condensed phase, the atomic states give rise to the free exciton states and to the trapped exciton states. The latter are considered to be identical to the excimer states, but shifted slightly in energy by weak interactions with the bulk. Arrows indicate the optical transitions brought about by the UV pump beam and the visible probe beam in the transient absorption experiments. 


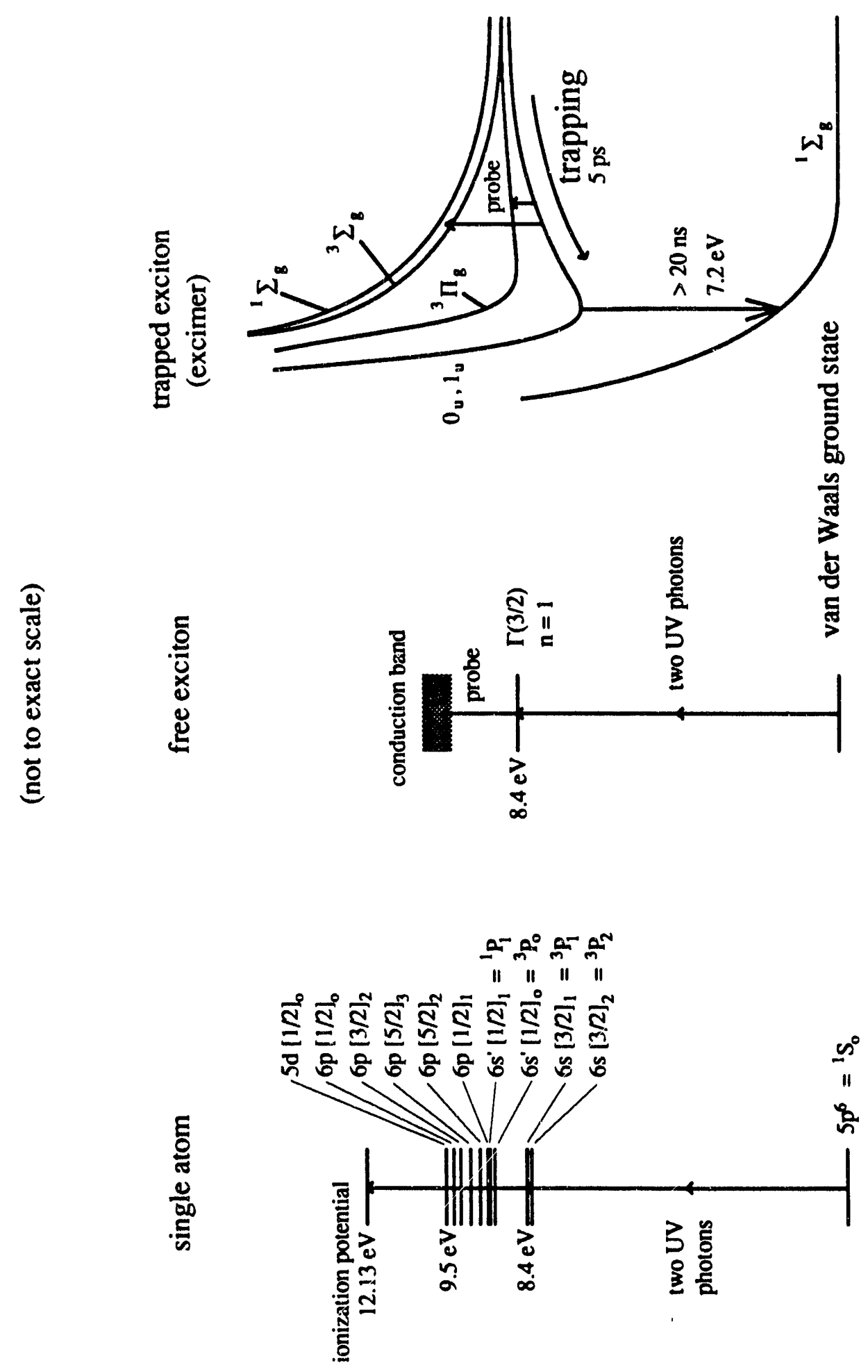

Figure 1: Xe atom, exciton, and excimer energy diagram xiv 
scale studying this process at cryogenic temperatures. This is the first study done at room temperature and also the first on the picosecond time scale. To my knowledge, no one has directly seen the trapping of the exciton under these conditions.

Herein is a description of the optical pressure cell that I designed and built to hold the liquid xenon near room temperature in an oil and other contaminant free environment. This is necessary as the machine oil commonly present in high pressure equipment readily absorbs the UV excite pulse and as such interferes with the dynamics both by displaying a transient absorption of its own and by acting as a quenching agent for the excited xenon species. Following this is the presentation of the xenon data, which exhibits a very interesting time dependence. The first few picoseconds contain sharp breaks, and at some wavelengths there is also a decrease in the transient absorption signal followed by an additional rise and then a subsequent longer time decay. We have modeled this as two coupled populations. The first, representing the free excitons, rises instantaneously and decays into the second population, which represents the excitons relaxing in the trapped state well. This second population rises with a time constant very similar to that with which the free excitons decay. The difference is due to a barrier between the free and trapped states and the cooling of part of the free exciton population within the free state 
well before crossing to the trapped state can take place. The cooled trapped excitons then undergo a longer time decay, representing the return of the population to the ground state. The resulting fits to the data suggest that this model is at least qualitatively correct. 


\section{Acknowledgements}

As with most gargantuan endeavors, many people have contributed to and have been helpful in my persuit of a Ph.D.

I would like to thank my advisor, Charles B. Harris, for providing the environment needed for good research: the freedon to persue one's ideas and the resources to do so effectively.

I must thank Vijaya Narasimhan, Charles' secretary, for her help in ordering equipment, for keeping the records of our accounts, for help in publishing papers, and in general for keeping the whole lab running smoothly. Things would have been quite a mess indeed without her administrative prowess.

I would like to thank my parents for their encouragement and support. Their love of learning and value of a good education has instilled the same in me and this has carried me far.

I would like to thank the professors at Gustavus Adolphus College, especially Dx. Larry Potts, Dr. Tom Gover, and Dr. Dick Fuller, who answered my hundreds of questions and persuaded me that I really should consider a career in research instead of going to medical school.

There are several fellow group members who have shown me how to do various things and who have helped with the taking of data. I would like to mention them here and extend a word of thanks to them. Mike Arndt showed me the ins and outs of 
working with an ultra high vacuum chamber and with timecorrelated-single-photon counting electronics. He and I took the tetracene data. He was also game for unlimited running stairs, lifting weights, and swimming. Dr. Michael G. Prisant assisted in obtaining some of the data for the rhodamine $B$ on silica system reported in part one of this thesis. Mark Paige instructed me on the use of the $10 \mathrm{~Hz}$ amplified picosecond system and high pressure equipment in return for my help in taking iodine data for his thesis. A good part of the xenon data was taken with the help of Ben Schwartz, and he was a part of the endless discussions that followed the data as we tryed to figure out what it all meant. Ben also was an excellent partner for a grueling bicycle ride as an outlet from the stresses of lab life. We rode together up nearly all the hills in the East Bay, Mt. Diablo, made an ill-fated attempt on Mt. Hamilton when it was $110^{\circ} \mathrm{F}$, and even did the infamous Markleeville Deathride. Walt Merry provide me with much needed help in machining and designing the high pressure cell used in the xenon experiments as well as in the task of filling this cell with xenon without contaminating the system. Walt also grew to be a close friend with whom I enjoyed hours bicycling, cooking and eating mounds of spaghetti, watching Twin Peaks, and drinking gallons of coffee and cappucinos, not to mention the memorable trip across the country when we moved Tsurumi to Rochester. Dan Russell wrote the program used to collect data and helped me implement it on the $10 \mathrm{~Hz}$ system. 
He was an irreplacable resource on computer information in general, and good for a rousing conversation on nearly anything at Romas.

There were others in the group that also contributed to my stay here, although I did not do experiments with them. Dave Smith helped me with computer stuff and was yet another partner on the bicycles. Robert Jordon also helped with the computers and joined walt and I many a time for riding and spaghetti. Jason $\mathrm{king}$ helped keep the $10 \mathrm{~Hz}$ system running and was always up for a science debate. Dave Padowitz, a post-doc, could always be engaged in a discussion on the life of a scientist in academics from every angle imaginable, and thus helped me sort out what I wanted to do for my post-doc. $H$ and $I$ also went slogging around in tide pools together, being both intrigued by the little slimy things that crawl there. Jennifer Hane lent an ear when I needed to talk about science an other things and provided an unmatched example of how to stay calm and collected throughout graduate school. I still don't know how she does this. Dee Pennington had us over for absolutely amazing Thanksgiving dinners for several years. Steve Gadd helped interpret some of the exciton theory papers with me. Robert Lingle, a new post-doc, was up for discussions of big biomolecules. With Jin zhang, another post-doc, I enjoyed many discussions on many topics especially the long one on the interaction of a photon field with matter. Several others influenced in one way or another my time here: 
Paul Alivisatos, Craig Parsons, Rebecca Hoff, and Karen Schultz.

I have had several good friends outside the group who I would like to thank as well. Robin McRae was a good roommate and partner for camping trips. Erich Schwiesow, another roommate, along with scot Kellar have tried to keep my obsessive ways of doing things that I get interested in under control. I've spent a good many times with them bicycling, backpacking, and just talking about stuff. Jeff Pelton is also a close friend from my days here at Berkeley; we have talked about all kinds of things, science and otherwise, at Romas and over e-mail. All four of these guys have been and continue to be great friends and have along with many in my group kept a note of human kindness and caring present in the sometimes mechanical and grueling environment of high-tech cutting-edge research.

I would also like to thank the folks at University Lutheran Chapel for the times spent there. The chapel was a good place to escape from science for a while and think about other important things in the world.

Finally, I want to thank Tsurumi Hamasu, my fiancee, for her love and support during the last three years. This has more than anything else kept me happy and sane during it all, in spite of being stuck apart for a good chunk of the time while she persued her MBA. She also helped me in setting up optics and in taking the first of the xenon data that 
demonstrated the feasability of the work described in the second half of this volume.

This work was supported by the National science Foundation. The U. S. Department of Energy, Office of Basic Energy Sciences, Chemical Sciences Division of the $U$. $S$. Department of Energy under contract number DE-AC03-76SF00098 is also acknowledged for some specialized equipment used in these experiments. 


\section{Chapter 1}

\section{Determination of Adsorbate Geometries on Surfaces Using a}

New Combination of second Harmonic Generation and Absorption

\section{Band Shifts}

\subsection{Introduction:}

A number of phenomena that occur at surfaces are sensitive to the geometry and orientation of adsorbate molecules. For instance, two dimensional energy transfer via dipole-dipole coupling varies both with intermolecular distance and with the relative orientation of the dipole mcments. Other examples include reactions and catalysis that take place on the surface which can only occur if the molecules assume a geometry correct for the reaction channel. Understanding these areas of chemistry, then, is contingent upon understanding the molecular distribution function of the adsorbates on the surface. In this chapter I would like to show how the combination of second harmonic generation (SHG) and a simple dipole-dipole interaction model can be used to gain insight into how molecules orient themselves relative to each other and to the surface. A subclass of possible molecular geometries can be defined from the overlap of the distributions predicted by each method alone.

Resonant SHG is able to define the angle between the transition dipole of an absorption band and the surface 
normal, but not the position of the molecule about this surface normal. SHg utilizing more than one resonance and thus more than one absorption band would allow determination of angles between two molecular dipoles and the surface normal. If these two dipoles are not collinear, then knowledge of their orientation is nearly equivalent to knowing the molecular orientation. At this point one need only decide which edge of the molecule is liable to interact with the surface.

Assuming that the molecules are positioned relative to the surface normal as dictated by the SHG results, the dipoledipole interaction model can be used to interpret shifts in the absorption bands of the adsorbate as a function of coverage in terms of the positions of the molecules relative to each other on the surface. A correction must be made, however, for the change in the dielectric constant and refractive index of the local environment of the molecule $s$ as they adsorb onto the surface from solution. Again, more complete information is obtained if shifts are measured for more than one absorption band.

\subsection{Theory for Analysis of second Harmonic Generation Data}

and Absorption Band shifts: SHG is used to determine the orientation of a molecular dipole relative to the surface normal. A laser beam of frequency $\omega$ and polarization $\hat{e}_{0}$ incident on a surface at an angle $\theta$ with respect to the 
surface normal will generate second harmonic with polarization $c_{2 .}$ and an intensity given by

$$
I_{2 \omega}=32 \pi^{3}\left(\frac{\omega^{2}}{c^{3}}\right) \sec ^{2} \theta\left|\hat{e}_{2 \omega} \cdot x_{s}^{(2)}(2 \omega=\omega+\omega): \hat{e}_{\omega} \hat{e}_{\omega}\right|^{2} I_{\omega}^{2} .
$$

Here the linear terms in the susceptibility of the surface have been neglected. $x_{z}^{(2)}$ is the second-order surface nonlinear susceptibility, and everything is in cgs units. Fresnel coefficients have been included in $\hat{\theta}_{6}$ and $\hat{\theta}_{20^{\circ}}$. If the SHG is primarily due to molecules adsorbed onto the surface and the molecular coverage is low, then one can ignore substrate contributions and local field effects and express $x_{8}^{(2)}$ as $^{6}$

$$
x_{s}^{(2)}=N\left\langle\alpha^{(2)}\right\rangle \text {, }
$$

where $N$ is the surface number density of the molecules, $\alpha^{(2)}$ is the molecular second-order nonlinear polarizability, and <> denotes an ensemble average over the molecular distribution. If the $S H$ frequency 20 is resonant with a transition from a nondegenerate ground state $|g\rangle$ to an excited state $|n\rangle$, then using time-dependent second-order perturbation theory one can express $\alpha^{(2)}$ as ${ }^{6}$ 


$$
\begin{aligned}
\alpha_{i j k}^{(2)}(2 \omega & =\omega+\omega)=-\frac{1}{\pi \hbar^{2}} \alpha^{(1)}(2 \omega)\left\langle g\left|p_{i}\right| n\right\rangle \\
& \times \sum_{n^{\prime}} \frac{\left\langle n\left|p_{j}\right| n^{\prime} X_{n^{\prime}}\left|p_{k}\right| g\right\rangle+}{\omega-\omega_{n^{\prime}}} \frac{\left.\ln \left|p_{k}\right| n^{\prime} X_{n^{\prime}}\left|p_{j}\right| g\right\rangle}{},
\end{aligned}
$$

where the nonresonant part of $\alpha_{i j k}{ }^{(2)}$ has been neglected, $\alpha^{(1)}(2 \omega)$ is the first order polarizability at $2 \omega,\left|n^{\prime}\right\rangle$ is an intermediate state, $p$ is the dipole operator, and $\omega_{n \text { 'g }}$ is the transition frequency between $\left|n^{\prime}\right\rangle$ and $|g\rangle$.

If the surface itself is isotropic with respect to the distribution of binding sites, then one would expect that the adsorbate layer would also be isotropic. In this case, the nonzero elements of ${ }^{s} \chi^{(2)}$ are ${ }^{s} \chi^{(2)}{ }_{222}{ }^{s} \chi^{(2)}{ }_{z i}$, and ${ }^{s} \chi^{(2)}{ }_{i z i}$ (changing the notation for clarity), where $i=x$ or $y$. Also in general ${ }^{s} \chi^{(2)}{ }_{i j k}={ }^{s} \chi^{(2)}{ }_{i k j}$. For a given laser incidence and polarization, a ratio, $R$, of $S H$ intensity from p-polarized excitation to $S H$ intensity from s-polarized excitation can be expressed in terms of various elements of ${ }^{s} \chi^{(2)}$. Rewriting Eq. (2) more explicitly, the ijkth element of ${ }^{s} \chi^{(2)}$ can be expressed as an average of the i'j'k'th element of $\alpha^{(2)}$ over the molecular distribution: 6

$$
{ }^{s} \chi_{i j k}^{(2)}=N \alpha_{i^{\prime} j^{\prime} k^{\prime}}^{(2)}\left\langle\left(\hat{\mathbf{i}} \cdot \hat{i}^{\prime}\right)\left(\hat{j} \cdot \hat{j}^{\prime}\right)\left(\hat{\mathbf{k}} \cdot \hat{\mathbf{k}}^{\prime}\right)\right\rangle,
$$


where the vectors in parentheses are unit vectors, the unprimed ones being in the $l a b$ frame and the primed ones being in the molecular frame. In order to evaluate the average $<>$, one must make some assumptions about the molecular distribution function. The dot products can be expressed in general using a set of Euler angles, say $\delta, \beta$, and $\gamma$.

The spectroscopy of dimers and higher order aggregates of molecules in which the wavefunction of each of the molecules is only slightly perturbed and thus retains an essentially monomeric form may be described with a simple dipole-dipole interaction model. 7,8 In the case of a dimer, which will be the dominant aggregate species at low coverages, the total wavefunction may be written as the product of the two monomer wavefunctions, $\psi_{1} \Psi_{2}$, and the Hamiltonian may be written as $H_{1}$ $+\mathrm{H}_{2}+\mathrm{V}_{12}$, where $\mathrm{H}_{i}$ is the Hamiltonian for an isolated molecule $i$ and $v_{12}$ is the interaction potential betwern molecules 1 and 2 . This interaction potential is most commonly approximated as a dipole-dipole interaction:

$$
v_{12}=\frac{\mu_{1} \cdot \mu_{2}-3\left(\mu_{1} \cdot \hat{I}_{12}\right)\left(\mu_{2} \cdot \hat{r}_{12}\right)}{r_{12}^{3}}
$$

Diagonalizing this Hamiltonian gives rise to a splitting of the excited state of the monomer:

$$
\Psi_{ \pm}=\frac{1}{\sqrt{2}}\left(\psi_{1}^{*} \psi_{2} \pm \psi_{1} \psi_{2}^{*}\right) \quad E_{ \pm}=E_{0} \pm V_{12},
$$


where * denotes the excited state wavefunction of the monomer and $E_{0}$ is the monomer excited state energy. Physically $\psi_{+}$ corresponds to exciting the transition dipoles of the two monomers in phase, while $\psi$. corresponds to them being excited out of phase. The magnitude of the splitting and the selection rules for transitions from the ground state are governed by the geometry of the dimer and the resulting oscillator strength. For parallel, in phase dipoles at angle $\theta$ to $r_{12}$, only transitions from the ground state to the excited state $\Psi_{+}$ are allowed. For $0^{\circ}<\theta<54.7^{\circ}$, is the lower state and an absorption red shift results, and for $54.7^{\circ}<\theta<90^{\circ}, \psi_{+}$is the upper state and an absorption blue shift results. Geometries in which the dipoles are not parallel have allowed transitions to both of the excited states, resulting in a splitting of the absorption peak into a red shifting portion and a blue shifting portion. If more than one monomer is in the aggregate, as will be the case at higher coverages, then the splitting becomes greater. For example, in the case of $\mathrm{N}$ parallel dipoles of magnitude $\mu$ arranged in a line separated from one another by distance $r$ and at angle $\theta$ with respect to this line, the splitting will be

$$
V_{12}=\frac{2(N-1) \mu^{2}\left(1-3 \cos ^{2} \theta\right)}{N r^{3}}
$$

It should be noted that the degree of additional splitting for 
each dipole added to the chain decreases since as $N \rightarrow \infty,(N-1) / N \rightarrow 1$.

To be more general, consider the case of two molecules on the surface forming a dimer in some arbitrary geometry. Let each molecule have transitions for which shifts in the absorption spectrum can be measured. Further, let the dipoles of these transitions be used to define a molecular coordinate axis labelled such that a given dipole, $\mu_{i}$, lies along the $i$ th axis of that molecule. Since the SHG will fix the orientation of each molecule relative to the surface normal, as described by Euler angles $\delta$ and $\beta$ mentioned above in the SHG formalism, the position of one molecule on the surface will differ from that of another only in terms of a rotation about the surface perpendicular. This rotation is simply the difference in the values of the remaining Euler angle $\gamma$ for the two molecules. The Euler angles can be defined as a series of consecutive rotations of the molecular frame $x^{\prime} y^{\prime} z^{\prime}$ to arrive at the lab frame $x y z$ while keeping the adsorbate molecules fixed, as shown in Figure 2 . These rotations will be about the expressed axis as viewed when looking along this axis from the positive direction into the negative direction. Let $\delta$ be a counterclockwise rotation about the molecular $z '$ axis to give the $x^{\prime} y^{\prime}{ }^{\prime} z$ '' system. Let $\beta$ be a counterclockwise rotation about $y$ '' to give the $x^{\prime \prime} y '{ }^{\prime} z '{ }^{\prime \prime}$ system. Finally, let $\gamma$ be the counterclockwise rotation about 2 '' to give the lab system xyz. For convenience, fix $\gamma=0$ for molecule one. The 
Figure 2: (a-c) Euler angles used to transform the molecular coordinate system $x^{\prime} y^{\prime} z$ ' to the lab system $x y z$. (d) Coordinates used to define the position of $\mu_{2}$ relative to $\mu_{1}$. 

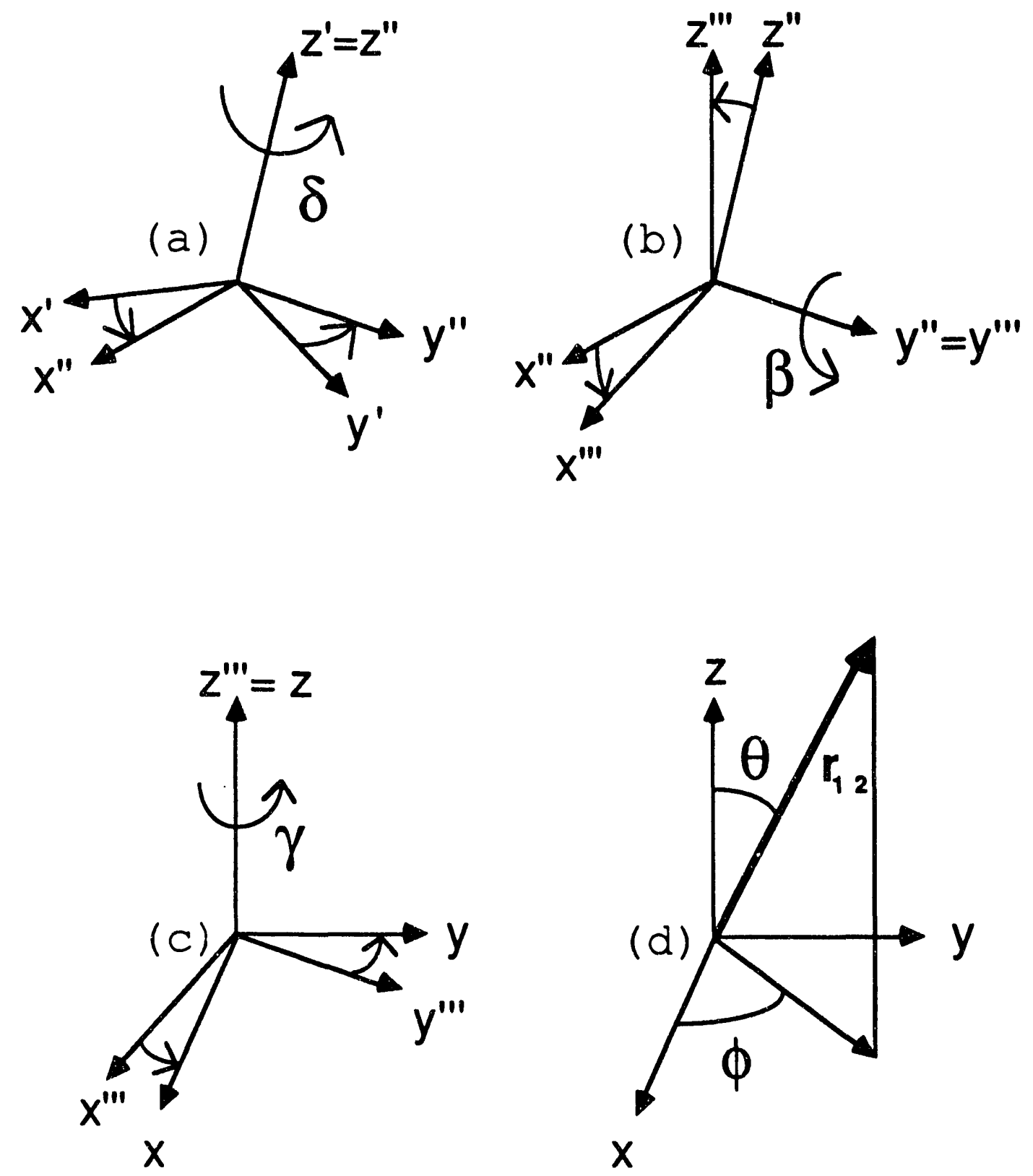

Figure 2: Coordinates for SHG and dipole equations 
rotation matrix for expressing $\mu_{i}$ of molecule one in the lab frame is then

$$
\left(\begin{array}{l}
\mu_{i x} \\
\mu_{i y} \\
\mu_{i z}
\end{array}\right)=\left(\begin{array}{ccc}
\cos \beta \cos \delta & \cos \beta \sin \delta & -\sin \beta \\
-\sin \delta & \cos \delta & 0 \\
\sin \beta \cos \delta & \sin \beta \sin \delta & \cos \beta
\end{array}\right)\left(\begin{array}{l}
\mu_{i x^{\prime}} \\
\mu_{i y^{\prime}} \\
\mu_{i z}^{\prime}
\end{array}\right),
$$

and the corresponding matrix for $\mu_{i}$ of molecule two is

$$
\left(\begin{array}{l}
\mu_{1 x} \\
\mu_{1 y} \\
\mu_{1 z}
\end{array}\right)=\left(\begin{array}{ccc}
\cos \gamma \cos \beta \cos \delta-\sin \gamma \sin \delta & \cos \gamma \cos \beta \sin \delta+\sin \gamma \cos \delta & -\cos \gamma \sin \beta \\
-\sin \gamma \cos \beta \cos \delta-\cos \gamma \sin \delta & -\sin \gamma \cos \beta \sin \delta+\cos \gamma \cos \delta & \sin \beta \cos \gamma \\
\sin \beta \cos \delta & \sin \beta \sin \delta & \cos \beta
\end{array}\right)\left(\begin{array}{l}
\mu_{1 x^{\prime}} \\
\mu_{1 y^{\prime}} \\
\mu_{1 z^{\prime}}
\end{array}\right) \text {. }
$$

Lastly, one can define a vector connecting the origins of the two molecular coordinate systems using spherical coordinates $\theta, \phi$, and $r_{12}$. Since neither SHG nor a dipole-dipole interaction model can distinguish between two molecules different distances from the surface, $\mathbf{r}_{12}$ must be restricted to be parallel to the surface, requiring that $\theta=0$. From equations (5), (8), and (9), the resulting energy shifts for the transitions associated with $\mu_{x^{\prime}}, \mu_{y^{\prime}}$, and $\mu_{z^{\prime}}$ are given by 


$$
\begin{gathered}
\Delta E_{x}=\frac{\mu_{x}^{2}}{r^{2}}\left\{\cos \gamma \cos ^{2} \beta \cos ^{2} \delta+\cos \gamma \sin ^{2} \delta+\cos ^{2} \delta \sin ^{2} \beta\right. \\
-3\left[\cos ^{2} \beta \cos ^{2} \delta \cos \phi \cos (\gamma+\phi)-\frac{1}{2} \cos \beta \sin (2 \delta) \sin (\gamma+2 \phi)\right. \\
\left.\left.+\sin ^{2} \delta \sin \phi \sin (\gamma+\phi)\right]\right\}
\end{gathered}
$$

$$
\begin{gathered}
\Delta E_{y}=\frac{\mu_{y}^{2}}{r^{2}}\left\{\cos \gamma \cos ^{2} \beta \sin ^{2} \delta+\cos \gamma \cos ^{2} \delta+\sin ^{2} \beta \sin ^{2} \delta\right. \\
-3\left[\cos ^{2} \beta \cos \phi \cos (\gamma+\phi) \sin ^{2} \delta-\frac{1}{2} \cos \beta \sin (2 \delta) \sin (\gamma-2 \phi)\right. \\
\left.\left.+\cos ^{2} \delta \sin \phi \sin (\gamma+\phi)\right]\right\}
\end{gathered}
$$

and

$$
\Delta E_{2}=\frac{\mu_{z}^{2}}{r^{2}}\left\{\cos \gamma \sin ^{2} \beta+\cos ^{2} \beta-3\left[\cos \phi \sin ^{2} \beta \cos (\gamma+\phi)\right\} .\right.
$$


These shifts can be used to predict a geometry for the dimer, and the resulting possible orientations of the molecules on The surface will be a distribution narrower than would have been predicted by either SHG or the absorption shifts alone. Due to a change in the refractive index and dielectric constant upon adsorption on the surface from a solution, the absorption spectrum of the adsorbate will undergo a shift in frequency. Several theories have been developed, most often using a classical reaction field that interacts with the transition dipole, to explain this effect and predict the magnitude of the shift.9,10 McRae has derived a general expression for the shift upon solvation from the vapor phase using second order perturbation theory incorporating both dispersive and static dipole interactions between solute and solvent molecules:

$$
\Delta v=(A L+B) \frac{n_{0}^{2}-1}{2 n_{D}^{2}+1}+C\left(\frac{D-1}{D+2}-\frac{n_{D}^{2}-1}{n_{0}^{2}+2}\right),
$$

where A, B, and C are solute dependent constants containing fundamental constants, an assumed cavity length, and static dipole moment magnitudes; $\mathrm{L}$ is a "weighted mean wavelength" of the solvent; $n_{0}$ is the refractive index at the sodium $D$ Iine; and $D$ is the static dielectric constant of the solvent. 11 Red shifts are negative in sign and blue shifts are positive 
in sign. An adsorbate on a surface will experience an environment that consists of the surface under it and the atmosphere over it. Values for $n_{D}$ and $D$ must therefore be some weighted average of values characteristic of the bulk solid and values characteristic of the atmosphere over the surface. In addition, as the surface coverage is increased, the additional adsorbate molecules present on the surface will cause a further change in $n_{D}$ and $D$, resulting in a coverage dependent shift. George and coworkers ${ }^{12}$ have utilized this coverage dependence of $n$ and $D$ to study shifts in the absorption spectrum of pyrene on $\mathrm{Al}_{2} \mathrm{O}_{3}$.

\subsection{Example Calculation of Rhodamine B Geometry on a Fused} silica surface and Discussion: To illustrate the utility of this combination of SHG with the dipole-dipole interaction model, I have examined a system of rhodamine $B$ adsorbed on a fused silica surface, simplified by utilizing sHG and shift data from only one absorption band. Shen and coworkers studied the orientation of rhodamine molecules on a quartz surface using resonant SHG. ${ }^{3}$ By using polarized light from a laser, one can select specific elements of ${ }^{8} \chi^{(2)}$ through which $\mathrm{SH}$ is generated. For a laser beam incident at forty-five degrees to the surface, the aforementioned ratio $R$ in terms of elements of ${ }^{s} \chi^{(2)}$ is $^{6}$ 


$$
R=\left|\frac{\left(\frac{1}{\sqrt{2}}\right)^{3}\left({ }^{s} x_{2 \times x}^{(2)}+s_{x_{z z z}^{(2)}}-s_{x_{x x}}^{(2)}\right)}{\left(\frac{1}{\sqrt{2}}\right)\left(s_{x_{2 \times x}^{(2)}}\right)}\right| \text {. }
$$

The intramolecular orientations of the transition moments for the rhodamine dyes have been determined previously. ${ }^{13}$ Ignoring the carboxyl group on the phenyl substituent attached to the xanthene skeleton, these dyes exhibit $c_{2 v}$ symmetry (see Figure 3). The rotation axis passes through the oxygen in the xanthene skeleton and the carbon-carbon bond between the xanthene skeleton and the phenyl group. For our purposes, label this rotation axis $z^{\prime}$ and the axis perpendicular to this lying in the plane of the xanthene ring system as $x^{\prime}$. The third axis, then, is $y^{\prime}$ and lies perpendicular to the plane of the molecule. The $S_{0}$ and $S_{2}$ states are even under reflection over the $y^{\prime} z^{\prime}$ plane, while the $s_{1}$ state is odd under this reflection. Therefore, the dipole moments for the $S_{0} \rightarrow S_{1}$ and the $s_{1} \rightarrow S_{2}$ transitions lie along the $x^{\prime}$ axis, and the dipole moment for the $S_{0} \rightarrow S_{2}$ transition lies along the $z^{\prime}$ axis. Since the transitions from $S_{0} \rightarrow S_{1}$ and from $S_{1} \rightarrow S_{2}$ are strong, it is reasonable to assume that the dominant term in the summation expressed in Eq. (3) above is the one with $s_{1}$ as $\left|n^{\prime}\right\rangle$. With this restriction, the summation contains only one nonzero 
Figure 3: Molecular coordinate system $x^{\prime} y^{\prime} z^{\prime}$ and rhodamine $\mathrm{B}$. 


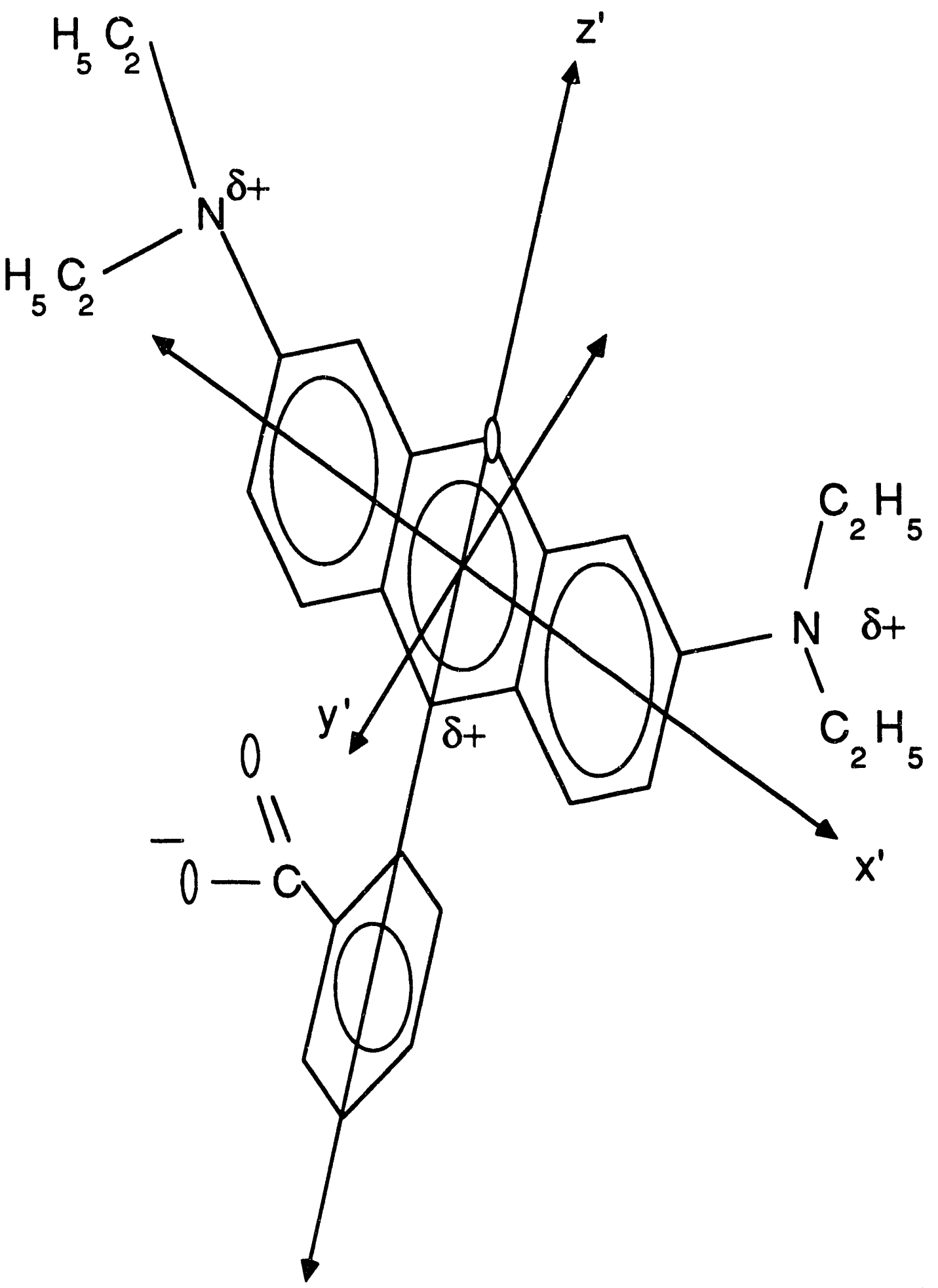

Figure 3: Rhodamine B and associated coordinate system 
term: $\alpha_{2(2)}^{\left(2 x^{\prime}\right.}$. Usi:ig Eq. (4) each element: of ${ }^{8} \chi^{(2)}$ can now be rewritten as

$$
s_{\dot{\chi}_{i j k}}^{(2)}=N \alpha_{z^{\prime} x^{\prime} x^{\prime}}^{(2)}\left\langle\left(\hat{\mathbf{i}} \cdot \hat{\mathbf{z}}^{\prime}\right)\left(\hat{j} \cdot \hat{\mathbf{x}}^{\prime}\right)\left(\hat{\mathbf{k}} \cdot \hat{k}^{\prime}\right)\right\rangle .
$$

In order to evaluate the average, it is necessary to make some assumptions about the molecular distribution function.

In writing Eq. (14) it has already been assumed that the molecules are randomly distributed in terms of their rotation about the surface normal. At this point, Shen and coworkers also assumed that the molecules are positioned such that the $y^{\prime}$ axis is parallel to the surface. Here that assumption has been relaxed and both $X^{\prime}$ and $Y^{\prime}$ have been allowed to be nonparallel to the surface. Evaluating the dot products in Eq. (15), inserting the results into Eq. (14), and performing an average of $\gamma$ over the range 0 to $2 \pi$ to achieve the desired isotropism about the surface normal, one arrives at a relationship between $\delta$ and $\beta$ for a given $R$ :

$$
\delta=\arccos \left(\frac{1}{\sqrt{\mathrm{K} s i n} \beta}\right),
$$

where $K=\left(R^{k}+1\right) /\left(R^{h}-1\right)$ and $\left|K^{h /} \sin \beta\right| \geq 1$. To evaluate the average denoted by $<>$, it has been assumed that all molecules lie on the surface at the same value of $\delta$ and $\beta$. From Eq. 
(16) it is apparent that there are many pairs of and $\delta$ and $\beta$ consistent with a given $R$. Shen and coworkers ${ }^{3}$ have experimentally determined $R \approx 2$. They then restrict $\delta=0^{\circ}$ and solve for $\beta=38^{\circ} .14$

Were $\mathrm{SH}$ data available for another set of transitions, say one involving $S_{2}$ as $\left|n^{\prime}\right\rangle$, another equation similar to Eq. (16) for another value of $R$ could be written. By solving these two equations, unique values for $\delta$ and $\beta$ would be obtained, which could then be directly substituted into equations (10), (11), and (12). For this application, since there are several possible values for $\delta$ and $\beta$, the problem is underdetermined and one cannot make full use of these equations to reduce the number of orientations of rhodamine $B$ on the quartz surface that are consistent with the sHg data. However, not all of the information contained in Eq. (16) has been extracted. Rearrangement of this equation gives

$$
\cos \delta \sin \beta=\frac{1}{\sqrt{K}}=\left\langle\hat{\mathbf{z}} \cdot \hat{\mathbf{x}}^{\prime}\right\rangle,
$$

in comparison to Eq. (8). This is simply the dot product between a unit vector parallel to the transition dipole for $S_{0}$ $\rightarrow S_{1}$ and the surface normal unit vector. Inverting Eq. (17) gives an expression for the angle between the dipole and the surface normal, $\xi$ : 


$$
\xi=\arccos \left(\dot{z} \cdot \hat{x}^{\prime}\right)=\arccos (\cos \delta \sin \beta)=\arccos \left(\frac{1}{\sqrt{K}}\right) \cdot
$$

Thus, one can say from the SHG how the transition dipole is oriented, but not how the molecule itself is oriented around this dipole. From Eq. (18) the dipole moment of interest is found to be at an angle of $52.02^{\circ}$ to the surface normal for $R$ $\approx 2$. The molecule itself can be rotated to any position about this dipole while still maintaining a geometry consistent with the SHG data, and in fact doing such will span all of the $\delta$ and $\beta$ pairs. With a value for $\xi$, the shift data can now be used to predict dimer geometries.

The absorption spectrum of rhodamine B has been taken for coverages between 0.3 and 1.5 monolayers, as determined from the measured absorption, assuming an area of $100 \AA^{2}$ for the molecule, and assuming that the molecules fill one monolayer completely before starting the next. The samples were prepared by simply laying a piece of Kodak lens tissue on the surface of a one centimeter quartz cuvette, placing a drop of a millimolar methanol solution of rhodamine $B$ on the tissue at one end of the cuvette, and drawing this drop across the face of the cuvette with the tissue. Coverages were more uniform with this method than with previous methods using centrifugation and were loosely correlated with solution concentration. Energy shifts in the $s_{0} \rightarrow s_{1}$ absorption peak due to 
the change in environment upon adsorption were measured with respect to the position of this peak at 545 nanometers for a $4 \times 10^{-6}$ molar methanol solution.

For the coverage range of 0.3 to 1.5 monolayers, an absorption red shift of 170 to $1050 \mathrm{~cm}^{-1}$ was observed with some broadening of the peak, but no sign of splitting, indicating that the dominant dimer geometry on the surface is one with parallel dipole moments (see Figure 4). Consistent with Eq. (7), the observed shift asymptotically approaches a value slightly greater than $1000 \mathrm{~cm}^{-1}$ with higher coverages. These results are consistent with those observed by Kemnitz et a). 15

Rather than use the general formalism of eq. (10), a formula that gives the shift explicitly as a function of $\xi$ will be used. Let the dipole moments of the two monomers in the dimer be in planes parallel to the lab yz plane at angle $\xi$ to the $z$ axis (see Figure 5 ). As before define $r_{12}$ in terms of the spherical coordinates $r$ and $\phi$ to be the vector joining the two molecular coordinate system origins. The resulting shift in the excited state of this geometry is

$$
v_{12}=\frac{\mu^{2}}{r^{3}}\left(1-3 \sin ^{2} \xi \sin ^{2} \phi\right)
$$

A surface adlayer constructed from dimers in the geometries proposed by Kemnitz et al.$^{15}$ in which both rhodamine 
Figure 4: Red shift in $S_{0} \rightarrow S_{1}$ absorption peak upon adsorption on the surface as a function of coverage (1's are our data, $x^{\prime}$ 's are Yoshihara's ${ }^{15}$ data with the molecular area rescaled to $100 \AA^{2}$ ). 


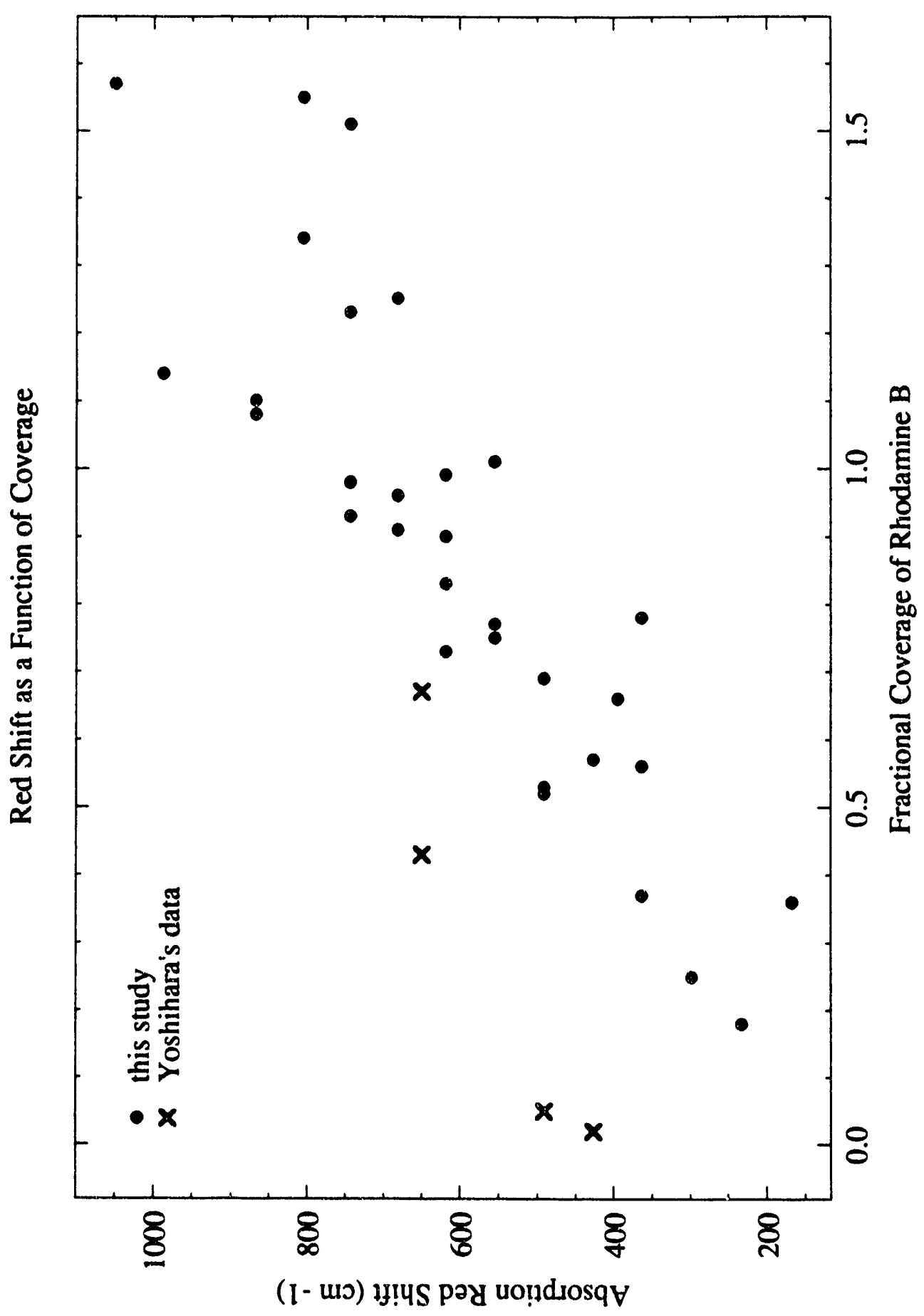

Figure 4: Absorption red shift for Rhodamine B on silica 
Figure 5: Simplified coordinate system to define the geometry of the dimer. 


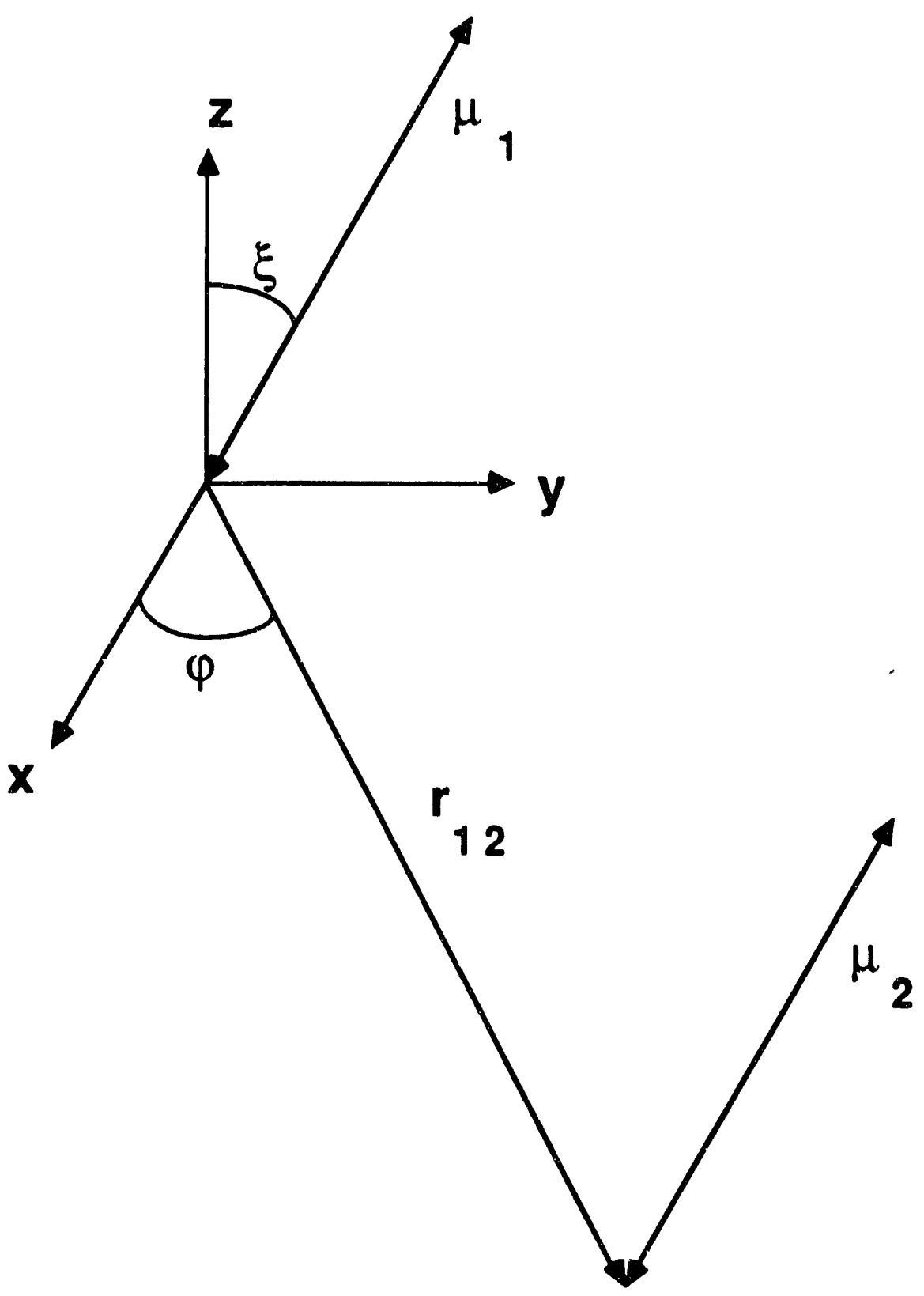

Figure 5: Coordinate system for dipole-dipole interaction 
molecules lie flat on the surface plane would result in a centrosymmetric dye layer, off which SHG would not be allowed. If instead the angle of the dipole with respect to the surface normal is taken to be $52^{\circ}$ as predicted by SHG, then several geometries consistent with the observed shifts can be constructed using Eq. (19). It is probable that the rhodamine molecules are interacting with the surface by forming hydrogen bonds between their amino and carboxylic acid groups and the surface hydroxyl groups. This is supported by nonresonant SHG performed on several different dyes by DiLazzaro et al. in which changes in the angle between the dye molecule and the surface normal are observed upon removal of the carboxylic acid group. ${ }^{16}$ For a dimer geometry to give only a red shift, the two dipoles must be parallel, and in the case of a symmetric molecule such as rhodamine $B$, the transitions must

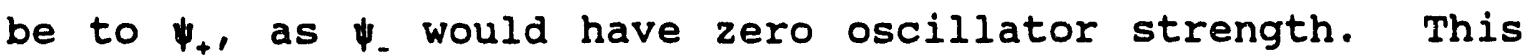
implies that $v_{12}$ must be negative, and restricting $\xi=52^{\circ}$ further implies that $\phi$ must have a value such that $47.1^{\circ}<\phi$ $\leq 90^{\circ}$

Even with these restrictions there are still many possible dimer geometries, shown in Figure 6 . It is well known that aromatic molecules crystallize such that pairs of molecules overlap in the unit cell. The geometry taken by the pair is often a " $T$ " or an offset stack in which a corner of one molecule's pi system sits close to the center of other molecule's pi system. The rhodamine molecules also have the 
Figure 6: Possible dimer geometries (omitting the amino anci carboxyl groups for clarity). 

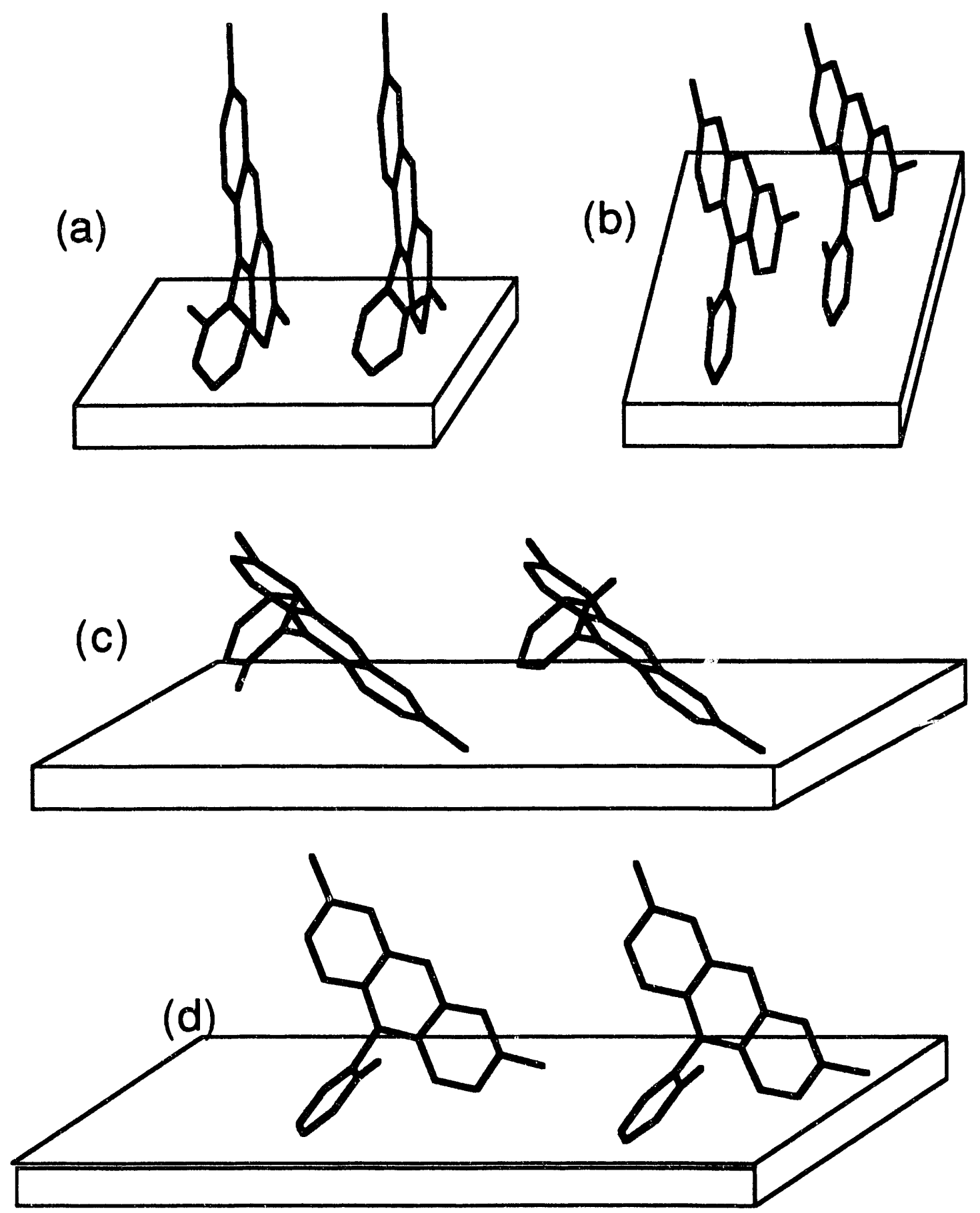

Figure 6: Dimer geometries for Rhodamine B on silica 
Table I: Red Shifts for Rhodamine Dimer Geometries

Energy shifts for geometries presented in Figure 6 for several values of $r$ and $\phi$.

\begin{tabular}{crrr}
\hline \hline Geometry & $r(A)$ & $\phi(\mathrm{deg})$ & $\mathrm{V}_{12}\left(\mathrm{~cm}^{-1}\right)$ \\
\hline a & 7 & 0 & 1716 \\
& 8 & 0 & 1149 \\
$\mathrm{~b}$ & 13 & 47 & 0 \\
& 13 & 60 & -106 \\
& 13 & 75 & -198 \\
$\mathrm{c}$ & 9 & 90 & -697 \\
& 10 & 90 & -508 \\
& 11 & 90 & -382 \\
& 12 & 90 & -294 \\
$\mathrm{~d}$ & 12 & 90 & -294 \\
& 13 & 90 & -231 \\
& 14 & 90 & -185 \\
\hline \hline
\end{tabular}


charged functional groups on their periphery. These interact with both the surface and with the other rhodamine molecules. In speculating about the likely dimer geometry on the surface all these factors must be considered. The shifts associated with several values of $\boldsymbol{r}$ and $\phi$ are shown in Table $I$. The smallest value of $r$ for a given geometry is that which brings the van der Waals surfaces of the two monomers into contact, as determined with molecular models. In geometries (b) and (d) the two monomers have their $x^{\prime}$ axes at $52^{\circ}$ to the surface normal and their $y^{\prime}$ axes parallel to the surface. These two geometries allow the carboxyl group and one of the amino groups to interact with the surface, and thus form stabilizing hydrogen bonds. Electrostatically (b) is more favorable than (d) since in (b) the negatively charged carboxyl group of one monomer is in closer proximity to the slightly positive amino group of the other. One can reduce the distance $r$ between the monomers, increasing the magnitude of the shift, by allowing the $y^{\prime}$ axes to be nonparallel to the surface, effectively rotating the monomers about their $x^{\prime}$ axes. This creates a geometry as depicted in (c). Such a geometry does, however, require that the carboxyl group of one of the monomers point upwards, away from the surface for small values of $r$. Lastly, geometry (a) is one that cannot exist on the surface, as it would give rise to a blue shift. It is also electrostatically unfeasible due to the proximity of like-charged functional groups on the two monomers. 
Comparison of the observed red shift (see Figure 4) wj.th the results in Table I reveals that the expected shift is less than that actually observed by $300-400 \mathrm{~cm}^{-1}$. At this point no correction has been made for the change in the dielectric constant and refractive index of the medium around the molecules upon adsorption on the surface. Kemnitz et al. have used McRae's formula, Eq. (13) above, to fit absorption shift data for rhodamine $B$ in several different solvents. ${ }^{15}$ From the fit they obtain values of $-9000 \mathrm{~cm}^{-1}$ for $(\mathrm{AL}+\mathrm{B})$ and -3424 $\mathrm{cm}^{-1}$ for $c$. They then estimate that the surface dielectric constant for quartz is approximately 70. This is much larger than what has been measured before for a quartz surface, but these surfaces are not clean--they have an overlayer of water adsorbed on them from being exposed to the air. The value of 70 , then, is quite reasonable, as it is close to the value of 80 commonly quoted for water itself. With these results, one predicts that the shift for rhodamine $B$ in methanol is -3970 $\mathrm{cm}^{-1}$ and that for rhodamine $B$ on quartz is $-4300 \mathrm{~cm}^{-1}$, or a red shift of $330 \mathrm{~cm}^{-1}$ in the absorption spectrum of rhodamine $B$ on going from methanol to the quartz surface. This then brings the experimental results into agreement with what is predicted by this simple model. To be more rigorous, one should include the coverage dependence of $n_{0}$ and $D$.

As one last point, more than two monomers can align themselves in these geometries in a chain-like fashion to produce a shift even greater than shown in Table I. The 
magnitude of this shift will be that of the dimer multiplied by $2(N-1) / N$, where $N$ is the number of monomers involved. For an infinite chain, the shift will be twice that of the dimer.

In the rhodamine on fused silica system, there are several levels of heterogeneity, and one might consider a multi-site model to explain the trends of the red shift as a function of coverage, rather than invoking aggregation as the cause. The surface of the fused quartz cuvette terminates in hydroxyl groups, to which an adsorbed layer of water and methanol (from the sample preparation) are hydrogen boncied. There would be a distribution of acidities amongst these sites, and each would have a different propensity for interacting with the rhodamine. In the methanol solution used to dose the surface, the rhodamine is a zwitterion, and so adsorption to the surface could occur through hydrogen bonds to both the negative carbaxyl group and the positive amino groups. The carboxyl group is known to be important in surface binding, as was stated above, and the positive charge delocalized throughout the xanthene skeleton would become localized on an amino group that partook in hydrogen bonding, thereby strengthening this interaction. It is not likely that both amino groups are hydrogen bonding, as this would result in a larger shift than is seen as the molecule attempts to construct two positively charged centers within itself. This would also tend to require that the molecule lay flat on the 
surface so that all three functional groups could bind, which would then lead to no SHG, which is not the case. So the most likely surface geometry is one with one amino group and the carboxyl group hydrogen bonded to the surface. Such a geometry would require that a basic and an acidic site be available or the surface separated by about the same distance as the amino and carboxyl groups on the rhodamine. At low coverages, the rhodamine would preferentially settle into these ideal sites, but as the coverage increased, the rhodamine would have to populate less energetically favorable sites. With this model, the red shift observed for low coverages would be large, and as the coverage increased, the red shift of the additional nolecules would decrease as unfavorable sites became populated. The absorption spectrum, then, would start as a narrow peak, similar to the very dilute solution spectrum, shifted far to the red. As coverage increased the spectrum would broaden to the blue and no additional red shifting would be seen. This is clearly not the case in the experimental data: very low coverages have a very small shift from that of the solution spectrum, and as the coverage is increased, the red shift also steadily increases. The peak does, however, broaden with increased coverage, and does so slightly more in the blue half than the red half, so there must certainly be a distribution of sites, but the dominant cause of the observed red shift is aggregation, not the heterogeneity of the sample. It would be an 
interesting comparison to repeat this experiment in vacuo on a cleaved quartz crystal, which would not have the adsorbed water layers. Such a surface would have a much more homogeneous site distribution, and the coverage dependence of the red shift could be assigned to aggregation effects with more certainty. Changes from what has been seen in these experiments could then be ascribed to numerous sites upon the surface.

As noted above, data from more than one absorption band would allow further constraints to be placed on the orientation of the rhodamine $B$ adsorbed onto the quartz. With one absorption band, SHG was able to define the angle between the transition dipole of this band and the surface normal, but not the position of the molecule about this dipole. SHG data utilizing another absorption band whose transition dipole is perpendicular to that of the first would allow the angle of this dipole to the surface normal to be determined as well, thus fixing the molecular axis relative to the lab frame except for a rotation about the surface normal. The dipoledipole interaction could then be used to interpret shifts in these absorption bands as a function of the distance between the two monomers in the dimer and angles describing rotations of one monomer relative to the other. Thus, the interpretation of the shifts and SHG provides detailed knowledge of the molecular distribution function on the surface. 


\section{Chapter 2}

\section{Brief Aistory of Excitons and Their Existence in Xenon}

2.1 Introduction: In this chapter I wish to put the work contained in the second half of this thesis into a scientific context on a historical level, and in the process communicate the motivation for doing the work. I will quickly summarize some of the highlights in the body of research on excitons and review the aspects which will lead to the questions I address in my research. The presentation is roughly in chronological order. The first section covers the concept of an exciton itself and how they are involved in the electronic structure of rare gas solids. The next section outilines the results of some of the early experiments that confirmed the relevance of the excitons in the description of rare gas excited states, particularly xenon, as it is the subject of my work in the second half of the thesis. I then move into work on the liquid phase and the search for excitons therein. This is followed by a discussion of the formation of excitons and the energetics and dynamics of this process.

A notable difference between the condensed phases, solids and liquids, and the gas phase is the constant interaction of the atoms and molecules within the material. such interactions manifest themselves in many ways in the properties of condensed matter, including the structure, mechanical and 
thermodynamic constants, and chemical reactivity. One aspect of the material that is key to all of these properties is the electronic structure. It is well known that as atoms and molecules come together in crystalline solids that the individual electronic states of the atoms/molecules combine to form the band structure characteristic of the lattice. Recently the bridge between the gas phase and solids, namely small clusters and "nanocrystals," has been studied in an effort to understand the details of the formation of these bands. In disordered systems, such as glasses and liquids, the loss of periodicity is accompanied by a partial loss of the band structure of the well ordered solid. Nonetheless, by studying the electronic states of these disordered systems, one can understand something of both their structure and the dynamics of processes within them.

A description of an excited electronic state of a solid or liquid must consider the great degeneracy of energy levels on the level of the atomic or molecular orbitals. The indistinguishability of the atoms makes it grossly inadequate to describe the excited state as a single excited atom. In this incorrect picture, a single excited atom would be strongly coupled to the others in the crystal, and there would be a large probability for this excitation to hop to an adjacent atom. This degeneracy amongst the atomic levels when properly addressed in the theory results in the band structure mentioned above. 
All of the features in an absorption or emission spectrum cannot be accounted for with transitions between the ground, or valance, band and the conduction bands. There are often states of intermediate energy that reside in the band gap. These are due to defects or surfaces in the crystal, or, in the case of the perfect bulk crystal, to correlations between the excited electron and the hole that is left behind. In the 1930's Frenkel developed the idea of an exciton in a theory for the electronic structure of crystalline argon. ${ }^{17}$ Interestingly enough, it was not until the 1960's that the technology necessary for obtaining the absorption spectra of the rare gases was developed and the ideas in the theory tested. The Frenkel model dealt with the equivalency of each site by characterizing a delocalized excited state of the lattice as a superposition of wavefunctions each of which had the excitation on a different atom. These wavefunctions were constructed from products of the atomic wavefunctions. Implicit in this model is the assumption that the excited state be of small radius so that the atomic wavefunctions accurately describe the amplitude of the excitation residing on a single atom. In the spectrum of such a system, the peaks correspond directly to excited states seen in the spectrum of a single atom. In the rare gas solids, however, the correlated electron-hole pair has a radius greater than a single lattice site, and the Frenkel model fails.

Later, Wannier developed a theory constructed from the 
opposite point of view--that the electron and hole pair could be viewed as a superposition of plane waves, thus characterizing the excitation as a superposition of Bloch functions. ${ }^{18}$ The wavepacket resulting from this superposition is more localized in space than a pure plane wave, and as such has a lower total energy due to the coulomb interaction of the electron and the hole. Indeed, the exciton state is the lowest electronic excited state of the pure crystal. The wavevectors used to describe the state are written as the difference of the wavevectors of the electron and the hole, and a reduced mass is constructed from the effective masses of the hole in the valence band and the electron in the conduction band. This approach most accurately describes electronic excitations that are larger than the lattice parameter, and gives rise to a hydrogen-like series of excited states which are not directly related to the excited states of the individual atoms in the lattice. The spectrum of the rare gas solids does indeed contain peaks not seen in the spectrum of individual atoms, but there are also peaks that are clearly related to atomic like states. Thus, it turns out that the Wannier model also does not completely describe the electronic structure of the rare gas solids, which have been characterized as having intermediate radius excitons. (For more detail on these exciton theories, the reader is referred to the original papers cited above, to an excellent book by R. S. Knox, ${ }^{19}$ and to a review article by Fugol'.${ }^{20}$ ) 
To describe the spectrum of the rare gas solids, then, one is left with attempting to modify the Frenkel model by using non-orthogonality corrections to overcome the fact that the excited state wavefunction needs to overlap the surrounding atoms or attempting to modify the wannier model by invoking central cell corrections that define a cell in the center of the Brillouin zone where more localized wavefunctions for the lowest energy state of the hydrogenic series $(n=1)$ are constructed. Both of these methods have been used in a number of calculations to determine the band structure for the rare gases. $21,22,23,24,25$

Not surprisingly, excitons also exist in molecular crystals, the theory of which was first worked out by Davydov. ${ }^{26}$ In such systems the structure within the unit cell of the molecules becomes important, influencing both the absorption and emission spectrum. Single molecule features in the spectra may shift in energy, split into two or more peaks, or change oscillator strength upon formation of the crystal. From these changes information can be obtained about the molecular geometry within the unit cell. It was this aspect of molecular excitons that was utilized in the first chapter to analyze the absorption band shifts of rhodamine $B$ on fused silica surfaces.

2.2 Early Experiments Confirm the Existence of Excitons: At about the same time that exciton theory was developing, 
experimental techniques necessary for the study of the rare gas solids emerged as well. Preparation of these solids requires either high pressures or cryogenic temperatures. The latter became possible, and several studies were done on solid rare gases condensed on cold tips or inside small ampules that had first been evacuated. Thus, the solid phase could be entered from either the gas or liquid phases. The other necessities for the study of excitons and electronic states of the rare gas solids are a source and detector of far UV light and the associated evacuated propagation path and transparent windows for the photons. These also became accessible experimentally during the 1960's.

The first spectra of solid rare gases were obtained by Schnepp and Dressler ${ }^{27}$ and Baldini.28 Baldini's work showed a distinct doublet corresponding to the spin-orbit split lowest atomic transition. For the thin xenon films at $21 \mathrm{k}$ these peaks appeared at 8.36 and $9.53 \mathrm{eV}$, as compared to the atomic lines at 8.43 and $9.57 \mathrm{eV}$ for xenon in the gas phase. At energies above both of these peaks were smaller strength transitions that did not correlate to anything in the gas phase spectra. Baldini assigned these to absorption due to creation of the $\Gamma(3 / 2)$ and $\Gamma(1 / 2)$ excitons (here $3 / 2$ and $1 / 2$ refer to the $j$ value of the hole).

Baldini has analyzed his spectra with the Wannier exciton model. The transition energies for the hydrogenic series can be expressed as 


$$
h v_{n}=E_{G}-\left(\frac{G}{n^{2}}\right) \text { and } n=1,2,3, \ldots
$$

where $E_{G}$ is the energy gap, $n$ is the principle quantum number, and the binding energy, $G$, and reduced mass, $\mu$, are given by

$$
G=\frac{\mu e^{4}}{2 \hbar^{2} \epsilon^{2}} \text { and } \frac{1}{\mu}=\frac{1}{m_{e}^{*}}+\frac{1}{m_{h}^{*}} \text {, }
$$

where $\epsilon$ is the bulk dielectric constant and $m_{e}{ }^{*}$ and $m_{h}{ }^{*}$ are the reduced masses of the electron and hole respectively. The radius of each of the excitonic states is simply

$$
r_{n}=\frac{\epsilon n^{2}}{\mu}
$$

Using the energies of the $n=2$ and $n=3$ excitons, Baldini calculated a value of $9.28 \mathrm{eV}$ for the energy gap and 0.86 for the binding energy. The atomic peak at $8.36 \mathrm{ev}$ does not fit the series as it has too small a radius to be fit well by the uncorrected Wannier model.

Lastly, Baldini suggested that perhaps some of the other peaks in his spectra are due to excitons from origins at other points in the Brillouin zone. This analysis was continued by Phillips, 22 who assigned the higher energy peaks near $10.3 \mathrm{eV}$ and above to excitons originating from the $I$ point in the 
Brillouin zone and to transitions to the various conduction bands.

In the mid $1960^{\prime} \mathrm{s}$, work by Jortner et al. examined the electronic states of simple liquids. ${ }^{29}$ They postulated that if the lifetime of an excited atomic or molecular state in the liquid exceeds the time necessary for a conformational change in the liquid surrounding the excited moiety, then the excitation might become trapped by a solvent relaxation that results in a lower overall energy for the system. In the rare gases, stable excited dimers, known as excimers, exist in the gas phase and are bound by $1 \mathrm{eV}$ or more. Jortner et al. suggested that in the liquid phase of the rare gases, where the excited state lifetimes are typically on the order of a nanosecond or longer, an excited atom would suffer many collisions with its neighbors while in the excited state, and therefore be able to form a stable conformation in the liquid as a consequence of energy loss in the relaxation. That is, the free wannier type exciton would become a trapped exciton in a state similar to the gas phase excimer state. This was supported experimentally by the similarity of the emission of these trapped excitons to the emission of the gas phase excimers.

This phenomenon of trapping by lattice conformational changes had also been seen in the isoelectronic alkali halides. The theories that utilized a rigid lattice to describe these systems failed. Theories in which the lattice was capable of being distorted and polarized were much more 
successful. ${ }^{30}$

2.3 Excitons in Liquids: Do They Exist? Reflection experiments by Beaglehole performed just above and below the melting point of xenon revealed that the liquid spectrum is very much like that of the solid, but with some additional broadening. ${ }^{31}$ The reflection spectra also show a close similarity to the absorption spectrum taken by Baldini. The rare gas liquids are the only liquids to date in which a wannier type hydrogenic series has been observed.

Jortner proposed a model to account for the broadening of the liquid spectrum relative to that of the solid which treated the excited electron as nearly free and the hole as a nearly stationary trapped site. ${ }^{32}$ He proposed that as long as the mean free path of the electron was greater than the circumference of the Wannier orbit, that the electron bound states would remain well defined. A reduction of the lifetime of these bound states over those in the solid is expected due to scattering of the electron off the disordered atoms of the liquid. As long as this scattering does not prevent closure of the orbit, the excited state will exist. The linewidths predicted by this model are consistent with the degree of broadening seen by Beaglehole, supporting the concept of a heavy hole with a light electron captive around it in a Rydberg like state.

Doped rare gas liquids studied by Jortner et al. further 
supported the idea of trapping of excitons at low energy sites.33,34,35,36 The dopants were either rare gases heavier than the solvent or small molecules. The atomic/molecular states of the dopants were lower in energy than the states of the solvent and provided the trapping sites for the exciton energy. In these systems both the small radius atomic-like states and the hydrogenic series of Wannier states are observed. The hole becomes localized on the impurity site, and the trapped state then undergoes transitions very similar in energy to those of the impurity species in the gas phase. The shifting of energy that does occur is a solvation effect of the matrix.

Further evidence for the trapping of excitons at low energy sites in the solvent was given by Kubota et al. in an ionization experiment. ${ }^{37}$ The ionization yield for liquid argon excited with high energy electrons emitted from the decay of radioactive ${ }^{207} \mathrm{Bi}$ was found to increase by $13 \%$ when xenon was present at a $1.6 \%$ relative concentration. The energy of the $n=1$ exciton in liquid argon is close to that of the exciton in the solid $(12.0 \mathrm{eV})$, but greater than the ionization energy of xenon in argon $(10.6 \mathrm{eV})$. Energy transfer from the argon excitons to the xenon atoms results in ionization of the xenon. The ionization of krypton in argon requires more energy $(12.5 \mathrm{ev})$ and would not be observed unless higher energy states in the argon were populated. Kubota observed no enhancement of the ionization yield with 
krypton dopants and posited that the higher excitons decay rapidly to the $\mathrm{n}=1$ exciton on a time scale faster than energy transfer to the krypton. This relaxation of the excitons to the $\mathrm{n}=1$ state occurs via a multiphonon radiationless process, and from the observed yields it is estimated to be several tens of times faster than the relaxation time of the $n=1$ state itself to form $\mathrm{Ar}_{2}{ }^{*}$.

Steinberger et al. have studied free excitons in liquid xenon with a number of techniques and have shown correlation of these states with the free excitons found in solid xenon. The Wannier model predicts that the oscillator strength be proportional to $\mathrm{n}^{-3}$. With the intensities of the $\mathrm{n}=1$ and $n$ $=2$ peaks, Baldini obtained a ratio of oscillator strengths of 9, in good agreement with the predicted value of 8 . The actual value of the oscillator strengths for the spin orbit split $n=1$ exciton lines has been measured as $f(3 / 2)=0.16$ and $f(1 / 2)=0.13$ by Steinberger et al, ${ }^{38}$ which are markedly different from the gas phase values of 0.26 and 0.24 for the atomic ${ }^{3} \mathrm{P}_{1}$ and ${ }^{1} \mathrm{P}_{1}$ states. ${ }^{39}$ If the $\mathrm{n}=1$ excitons are well approximated by the Frenkel model, that is if they are of a radius not much larger than that of the excited atom, the values for the oscillator strengths of the excitonic and atomic transitions would be expected to be very similar. The notable difference found experimentally supports the conclusion that the excitons must have a larger radius. However, using the Wannier model to calculate the energy of the $n=1$ 
exciton from values for $E_{G}$ and $G$ derived from excitons with $n$ $>2$ results in a value that differs from the experimental value by 2 percent. These errors indicate that the $n=1$ state is indeed intermediate between the descriptions given by the Frenkel and Wannier models. Furthermore, Steinberger has calculated a value for the oscillator strength using a slater function for the ground state and a Wannier type function for the excited state. The result of $f=0.254$ is in fair agreement with experiment.

Steinberger et al. have also observed that the width of the free exciton state observed in reflection studies of pure samples is much greater than that of the trapped exciton dopant state seen by Jortner et al.40 They argue that the lineshape should be governed by the degree of scattering of the electron in the Bohr orbit of the exciton: sharper bands should correspond to longer relaxation times. The free exciton consists of a correlated electron and hole, each of which has a relaxation time associated with its motion; the smaller of the two relaxation times governs the linewidth. For the trapped exciton the correlation time of the electron governs the linewidth. The mobility of a particle is related to its correlation time by the simple formula $\mu=\left(\mathrm{e} / \mathrm{m}^{*}\right) \tau$. Free electrons in both liquid and solid xenon have a mobility of the order of $10^{3} \mathrm{~cm}^{2} / \mathrm{V}-\mathrm{sec}$, while the mobility for holes was not measurable in the liquid and found to be $0.02 \mathrm{~cm}^{2} / \mathrm{V}-\mathrm{sec}$ in the solid, ${ }^{41}$ indicating that tne relaxation time for the hole 
in the trapped exciton is much smaller than that for the associated electron. Since the relaxation time for the electron in the trapped exciton is greater than the relaxation time for the hole of the free exciton, the trapped excitons have sharper lines than the free excitons.

The relationship of the excitonic bands in the liquid to those in the solid has also been studied by steinberger et al. They varied the temperature from $6 \mathrm{~K}$ to $160 \mathrm{~K}$ and noted changes of band gap, binding energy, and effective mass within the Wannier model.42 plots of the peak position in the reflection spectra for the $\Gamma(3 / 2) \mathrm{n}=1,2$, and 3 and for the $\Gamma(1 / 2) n^{\prime}=1$ peaks versus temperature show a linear temperature dependence for the solid phase data, but the liquid xenon data points do not lie on these lines. All four peaks do, however, show a red shift with increasing temperature. When these same data are replotted as a function of density, all of the data, including that of the liquid, lie on a straight line. This result demonstrates that the excitons in the liquid are in fact related to those in the solid and that the density is a larger determining factor in the exciton energy than the degree of order within the media.

Using the energies for the $n=2$ and $n=3$ peaks, steinberger et al. calculated and plotted the binding energy, $G$, the energy gap, $E_{G}$, and the mobility, $\mu$, as a function of density. As the density increased, $G$ was found to decrease linearly, while $E_{G}$ and $\mu$ were found to increase linearly. 
Since the $\mathrm{n}=3$ exciton had not been observed in the Iiquid, a value for $E_{G}$ was obtained from the solid line using the liquid density and then used to calculate $G$ and $\mu$ values for the liquid. The resulting $G$ value was collinear with the solid data, but the $\mu$ value was much lower. The lack of a temperature independent region below the Debye temperature (55 $K^{43}$ ) indicated that scattering of the excitons by phonons was not a large factor in the observed red shift. This led steinberger et al. to propose that the shifts were due to dilation of the lattice with an increase in temperature. The same effect was observed by Coletti and Bonnot in all the rare gases for the free exciton (the $8.35 \mathrm{eV}$ peak in xenon Iuminescence), and they also ascribe the observed linear temperature dependence to an expansion of the lattice. ${ }^{44}$

The theory of deformation potentials can be used to interpret changes in the band gap as well as the accompanying changes in the excitonic peak positions in the spectra. ${ }^{5}$ From their linear plots of band gap versus density, steinberger et al. determined that $E_{10}$, the change in the band gap per unit dilation of the lattice, has a value of $-0.25 \mathrm{eV}$. Changes in the binding energy were assumed to be mainly due to a change in the dielectric constant with density, and changes in the mobility were not interpreted.

The most important point of this density study by steinberger et al. is the evidence that the excitons in the liquid are most definitely related to those in the solid, suggesting 
that it is the interatomic spacing that is the dominant factor in determining the excitonic electronic properties, not the periodic structure.

Steinberger et al. have followed up the reflectivity studies with photoconductivity experiments in order to independently ascertain values for the band gap, the binding energy and the effective exciton masses. The values obtained confirm that parameters for the liquid do in fact agree with extrapolations of the values of the solid to the liquid density region: ${ }^{46}$

parameter

$E_{G}$

G

$\mathrm{m}^{*}$ liquid value

$9.22 \mathrm{eV}$

$1.08 \mathrm{eV}$

0.31 extrap. solid value

$9.24 \mathrm{eV}$

$1.21 \mathrm{eV}$

0.27

The agreement of these values for the two phases further emphasizes the idea that it is the proximity of the atoms to one another that is important for the energetics of the excitons. Steinberger et al. also calculate other parameters using the above results in conjunction with literature values:

\begin{tabular}{lcc} 
parameter & liquid value & solid value \\
\cline { 2 - 2 } $\begin{array}{l}\text { momentum relaxation time } \\
\text { mean free path for } \\
\text { momentum transfer }\end{array}$ & $0.8 \mathrm{ps}$ & $0.34 \mathrm{ps}$ \\
scattering length & $710 \AA$ & $330 \AA$ \\
& $0.38 \AA$ & $0.42 \AA$
\end{tabular}


The similarity of these values further underscores the similarity of the excitonic behavior in the solid and liquid phases of xenon.

The primary focus of the foregoing pages in this section has been devoted to the history of the discovery of excitons in rare gas liquids and to the confirmation that they are indeed related to the same excitations that have been called excitons in rare gas solids. In the following, I will cover the work done on determining the sites of exciton formation in the liquid and solid, the relationship of excitons to other excitations, and the dynamics of the excitons during their formation and subsequent relaxation. Work in these areas has been put forth in the last two decades.

\subsection{Exciton Formation: Energetics and Dynamics: since} excitons are delocalized entities, the question of how they form and evolve as the rare gas goes from a gaseous state to a condensed state is important. Do the excitons evolve gradually from some state in the gas phase, or do they appear in coexistence with gas phase states and grow in intensity as the density is increased, while at the same time the gaseous states decline? The density dependence of exciton behavior in the liquid has been studied by steinberger et al., and the results indicate that although the parameters for the wannier description of the solid extrapolate to the liquid values with the change in density, the density dependence of the liquid 
spectra themselves differs from that of the solid.47,48 This suggests that different sites are present simultaneously in the liquid; whereas, in the solid each atom is in a rather well defined environment. The experiments were done above the critical point $\left(T=16.6^{\circ} \mathrm{C}\right.$ and density $=185.2$ amagats $\left.^{1}\right)$ so as to probe a continuous transition from gas to liquid densities without undergoing a phase transition. Transitions were observed to the $6 s(3 / 2),{ }^{0}, 6 s^{\prime}(3 / 2){ }_{1}^{0}$, and $5 d(3 / 2),{ }^{0}$ states over a density range of 80 to 457 amagats $(6 \mathrm{~s}$ and 5 d refers to the orbital of the excited electron, $3 / 2$ to the j-state of the hole, 1 is the total $\mathrm{J}$ value, 0 indicates a symmetric state, and the ' indicates spin-orbit states). In the solid phase, these transitions become the $n=1$ states of each of the exciton series, and they shift to the red with increasing density. In the liquid they also shift to the red with increasing density; however, this shift is accompanied by the growth of a broad hump around each peak, with most of its intensity to the red side of the solid transition energy.

Previous studies of the liquid referenced above were near the triple point (523 amagats) or the liquid-solid coexistence curve, where the liquid structure is expected to be reminiscent of the solid. The two papers discussed here indicate that excitonic states exist at densities far below that of the

'The amagat is a unit of density corresponding to the number density of the substance at STP, or the inverse of the molar volume times Avogadro's number. For xenon one amagat is $2.7067 \times 10^{19}$ atoms $/ \mathrm{cm}^{3}$. 
solid. Steinberger et al. analyzed their reflection spectra using a model incorporating Lorentz oscillators for the observed peaks, and they derived values for the optical constants $n, k, \epsilon^{\prime}$, and $\epsilon^{\prime \prime}$ and also the relative populations of each excitation created. They then suggested that density fluctuations in the liquid result in what might be described as "gaslike" sites and "condensed phase" sites. The main atomic peaks are from atoms that reside in a low density region, while the broad red hump corresponds to atoms that are in little clusters which have a well packed order and are thus more like a dense liquid or even the solid. To support the exciton as it might exist in the solid, a cluster of atoms in the liquid must be large enough to contain the Wannier orbit. At a given density, there should be a statistical population of clusters of atoms of various sizes. As the density increases, the possibility that a larger cluster will form and live long enough to support the exciton also increases. From the relative intensities of the gaslike and excitonic lines and a Gaussian model of density fluctuations, steinberger et al. calculated that for the formation of the $\Gamma(3 / 2) n=1$ exciton a critical cluster size exists in which 10 atoms fill a volume of $1500 \mathrm{~A}^{3}$, corresponding to a local density approximately half that of the triple point density. If this were a spherical volume, its radius would be $7.1 \AA$, larger than the 2.7 A exciton radius, but between the first two peaks of the pair correlation function, $g(r)$, at $4.34 \AA$ and $7.92 \AA .49$ 
The idea of a critical density for the appearance of the excitons would suggest that the excitons with a larger radius (higher $n$ values) would appear at a higher density than the smaller radius excitons. This is born out by the order of appearance of the $\Gamma(3 / 2) n=1$ and $n=2$ excitons and the $\Gamma(1 / 2) n=1$ exciton:

$\begin{array}{lcc}\text { exciton } & \text { radius } & \text { appearance density } \\ \Gamma(3 / 2) n=1 & 2.7 \AA & 100 \text { amagats } \\ \Gamma(1 / 2) n^{\prime}=1 & 4.2 \AA & 180 \text { amagats } \\ \Gamma(3 / 2) n=2 & 11 \AA & 400 \text { amagats }\end{array}$

Möller has seen similar size effects in the study of argon clusters, where surface and bulk excitons do not appear until the cluster reaches a critical size.50,51

Various excitation sources have been used to create excitons in liquids. The early experiments used arc lamps or the MeV electron emission from the radioactive decay of ${ }^{207} \mathrm{Bi}$. The hot electron source actually causes several processes to occur: primary and secondary ionization, as well as excitation to many of the electronic excited states, not just the lowest one. In such a case it has been commonly assumed that the exciton would form either from relaxation of the electronically excited atom to the lowest excited states via a combination of radiative and nonradiative transitions, which result in the concomitant launching of acoustic phonons, or 
that it would form from the recombination of an ion with one of the primary or secondary electrons emitted, and that the resulting species would electronically relax via the same mechanisms as the hot atoms. Kubota et al. have measured the quantum yield of luminescence from both self-trapped excitons and excitons formed by recombination of electrons and ions. 52 By applying an external electric field across the excited region, they removed the free carriers before they could recombine, and so the collected luminescence must have come from self-trapped excitons. In liquid xenon, the application of a $12.7 \mathrm{kV} / \mathrm{cm}$ field, which collected $99 \%$ of the charges produced, resulted in a decrease in the luminescence intensity by a factor of $74 \%$. From the luminescence intensity at a variety of applied field strengths, they calculated that the ratio of excitons to electron hole pairs produced by the hot electrons is 0.06 , and that the ratio of quantum efficiencies of the emission from excitons that are formed initially to those resulting from electron recombination is 5 . This implies that ions in the fluid can capture electrons and then form excitons, but that it is five times more likely that luminescence results from the initially formed excitons than by the product of the recombination process.

The exciton luminescence itself has been the subject of many studies in both the frequency and time domains. It contains information about the extent of energy relaxation experienced by the excitons during trapping, the number of 
different sites within the liquid and solid that act as traps, as well as the time scales for the trapping and final decay back to the ground state. In addition several theories have been developed to describe the mechanisms of trapping and relaxation, most often using a multi-phonon coupling scheme.

Kink, Lôhmus, and Selg have studied the luminescence spectrum of xenon in the 6 to $9 \mathrm{eV}$ region over a temperature range of about 5 to $150 \mathrm{k} \cdot{ }^{53}$ They find that the emission contains a narrow peak at nearly the same energy $(8.35 \mathrm{eV})$ as the absorption to the $\Gamma(3 / 2) n=1$ exciton as well as two broad bands at 7.2 and $7.6 \mathrm{eV}$. The broad bands were much stronger than the narrow band. It was also noted that while the main portion of the broad bands were Gaussian in profile, the low and high energy tails of each were much too intense to fit such a distribution. This excess intensity in the tails was attributed to emission from the turning points of excited vibrational states of the trapped excitons. The narrow band near the $8.35 \mathrm{eV}$ absorption frequency was attributed to the free exciton, that is the exciton as it exists before any trapping or lattice relaxation occurs. The 7.2 and $7.6 \mathrm{eV}$ bands were both assigned to trapped excitons associated with the ${ }^{3} \mathrm{P}_{1}$ state of the $\mathrm{Xe}_{2}{ }^{*}$ molecule. This assignment was based on the time decay of the two bands. Excitation spectra and the temperature dependence of these bands has indicated that they belong to different sites in the xenon, and kink et al. assigned the $7.2 \mathrm{eV}$ band to excitons trapped at a lattice 
vacancy defect, while the $7.6 \mathrm{eV}$ emission was assigned to excitons trapped in the bulk fcc lattice.

Martin has proposed that the temperature dependence of the luminescence is due to emission from the lowest excited electronic states of $\mathrm{Xe}_{2}{ }^{*}$, which consist of two separate groups that correlate to the various $J$ states of the lowest excitations in the atom. ${ }^{54}$ These two groups are separated by approximately $0.5 \mathrm{eV}$. Martin posited that the luminescence at 7.2 and $7.6 \mathrm{eV}$ come from these two groups of excited states. As the temperature is increased, the emission coming from the lower group increases at the expense of that from the upper group. His conclusion was based, however, on an incomplete temperature study in which the lowest temperature was $65 \mathrm{~K} .55$ At still lower temperatures, as described below, the reverse trend is true.

The temperature dependence of the luminescence is quite unique and has inspired many theorists to model the trapping behavior of the excitons in xenon. Kink et al. found that below about $55 \mathrm{~K}$ the emission occurs primarily at $8.35 \mathrm{eV}$ from the free excitons and at $7.2 \mathrm{eV}$ from the excitons trapped at defect sites. From $55 \mathrm{~K}$ to about $120 \mathrm{~K}$ the emission occurs primarily only at $7.6 \mathrm{eV}$ from the excitons trapped at bulk lattice sites. Above about $120 \mathrm{~K}$ the $7.2 \mathrm{eV}$ emission once again dominates the spectrum, but without any of the $8.35 \mathrm{eV}$ emission from the free excitons.

The large luminescence stokes shift of over $1 \mathrm{eV}$ has two 
contributing factors. Since the equilibrium bond distance of the excimer, $2.85 \AA$, is less than the equilibrium distance of two ground state atoms, $4.4 \AA$, the transition from the excited state to the ground state puts the molecule approximately $1 \mathrm{eV}$ up the repulsive wall of the ground state surface.56,57 The remaining portion of the stokes shift is the binding energy of the excited state well at the internuclear separation from which the molecule emits. This has been commonly estimated to be $0.5 \mathrm{eV}$ at the bottom of the well. Theories modeling the trapping try to describe pathways by which this $0.5 \mathrm{eV}$ can be dumped into the surrounding liquid or solid within the radiative lifetime of the exciton.

Some other details that must be considered when modeling the trapping process include the direction of the molecular bond within the lattice and the degree of lattice distortion that accompany the formation of the trap. The formation of color centers in alkali halide crystals is the same process as the formation of the trapped exciton by two xenon atoms. For the fcc alkali halide crystals, it has been shown that the color center consists of an electron bound to a trapped hole, which in turn is aligned with the [110] crystal plane. ${ }^{58,59}$ Likewise, this electron in the rare gas solids is in a Rydberg type state about the ion dimer, $\mathrm{R}_{2}{ }^{+}$, and most, if not all, of the relaxation energy arises from the formation of the molecular bond, and not the relaxation of the surrounding lattice. ${ }^{60,61}$ That the electron is in a Rydberg state around 
the ion also implies that the shape of the $R_{2}^{+}$molecular surface is nearly the same as that of the $R_{2}^{*}$ surface. These details, as will be explained further below, are why the luminescence from the trapped states exhibits a lineshape close that of the excimer in the gas phase.

Martin has modeled the trapping process in rare gas crystals using a resonantly enhanced energy transfer mechanism. ${ }^{54}$ He assumed weak coupling between the relaxing exciton oscillator and the lattice, and accounted for this in a single phonon mode perturbation expansion wherein the lattice atoms do not deviate markedly from the bottom of their harmonic wells. For an fcc lattice of atoms only acoustic phonons exist, and of these only longitudinal phonons along the [110] direction couple to the exciton molecular trap. Morse potentials were used to model the exciton molecule trap. For. the $\mathrm{Xe}_{2}^{*}$ molecule the vibrational level spacing at all points in the well except for the very top exceeds the energy of a single phonon at the Debye frequency, and as a result single phonon coupling cannot account for the observed relaxation to the bottom of the well. This implies that a second mechanism may be responsible.

Martin proposed a hopping mechanism wherein the collision of the molecule with a neighboring atom in the lattice results in a loss of energy via transfer of kinetic energy. The colliding neighbor is treated as being on the outer part of another Morse potential between itself and the atom in the 
molecule that it hits. As the collision drives the neighbor close to this atom in the molecule, the original molecular bond breaks and the atom forms a new bond with the incoming neighbor. This break occurs when the distance between the atom undergoing collision is equidistant from the collision partner and its original bonding partner. Energy analysis reveals that the new molecule is lower in its well by $0.75 \mathrm{E}_{\mathrm{k}}$, were $E_{k}$ is the kinetic energy of the original molecule. This is due to the release of the old bonding partner with $0.5 E_{k}$ and the equipartitioning of the remaining $0.5 \mathrm{E}_{\mathrm{k}}$ between the kinetic and potential energy of the new molecule. The center of mass of the new molecule continues to travel in the same direction as that of the original molecule and the process repeats itself until thermal energy is insufficient to drive a collision partner close enough to allow the energy transfer to occur. This is similar to the model used by Sun and Rice to describe the relaxation of $\mathrm{N}_{2}$ in an argon lattice, where the relaxation is found to be faster than the radiative lifetime in spite of the large discrepancy between the lattice phonon energy and the vibrational spacing of the molecule. 62 The results of Martin's calculation indicate that greater than $0.5 \mathrm{eV}$ of energy is released by the relaxing exciton into the lattice within six collisions. For xenon at $50 \mathrm{~K}$ and at 150 $K$, the process takes less than 4 picoseconds. As a conservative estimate, Martin proposed that all the rare gas excitons will be fully trapped within 6 picoseconds. 
Kink, Lôhmus, and Selg have analyzed their temperature data (see above) utilizing a vacancy model.63 They first refute some earlier icieas of the source of: the 7.2 and $7.6 \mathrm{eV}$ emission. Since in the gas phase the xenon excimer emits at $7.2 \mathrm{ev}$, they conclude that the $7.2 \mathrm{ev}$ emission from the crystal emanates from the lowest vibrational level of the trapped exciton state. The 7.2 and $7.5 \mathrm{eV}$ emission have similar lifetimes ( 20 and 15 nanoseconds respectively) so they must both be from the lowest triplet state of the exciton. The other spin-orbit state, a singlet, should have a much shorter lifetime. Finally one of the bands cannot be coming from the top of the exciton well and the other from the bottom as they both have similar Gaussian profiles. So the authors maintain their earlier supposition that the $7.2 \mathrm{eV}$ emission comes from an exciton localized at a crystal defect site, while that at $7.6 \mathrm{eV}$ comes from the excitons localized in the bulk.

since there is some crystal reorganization involved in the trapping process, Kink, Lômus, and selg suggest that the free excitons and the self trapped excitons are separated by a barrier, the size of which will depend on the exciton-phonon coupling. The exact energy of the $7.2 \mathrm{eV}$ band depends on the crystal preparation and annealing history. For crystals grown from pure gas samples, the majority of the defects should be vacancies or similar crystal disorders. At low temperatures, the concentration of the vacancies will be fixed, frozen into 
the crystal structure, but at a high enough temperature thermal energy will be adequate to overcome the enthalpy of formation of a vacancy. Thus at low temperatures the emission is from either the free state at $8.35 \mathrm{eV}$ or at $7.2 \mathrm{eV}$ from the few excitons that have found a frozen vacancy and become trapped there. As the temperature is increased, the free excitons can all surmount the barrier, most become trapped in the bulk lattice, and later emit at $7.6 \mathrm{ev}$. At still higher temperatures vacancies can form thermally and nearly all of the excitons find them, become trapped, and again emit at 7.2 ev. A thermodynamic model incorporating these ideas yields a barrier height of $80 \mathrm{meV}$ for transition from a free exciton to a self trapped exciton in the bulk and a vacancy formation enthalpy of $150 \mathrm{meV}$. These values are of the same order of magnitude as others published previously.

As a further test of their bulk/vacancy site model, Kink, Lôhmus, and selg calculated the relative energies of an exciton positioned in the bulk and at a vacancy. For the bulk case, the molecular trapped exciton was constructed by moving two adjacent atoms in the crystal plane together, while for the vacancy case, the trapped exciton was formed by moving atoms on opposite sides of the vacancy together. Using Morse potentials for the excitons and simple pair potentials for the other interactions with the lattice, they calculated a difference in the equilibrium bond distance of the excitons of $0.1 \AA$. This resulted in a difference of energy of 0.5 to 0.6 
eV for emission to the ground state, half of which was due to the difference in energy of the two points on the steep repulsive wall of the ground state to which the states emit.

A detailed calculation of the barrier heights and tunneling rates through the barrier involved in the trapping process for all of the solid rare gases has been published by Kmiecik and Schreiber. ${ }^{44}$ This work is a modified version of the quasi-classical theory of Nasu and Toyozawa. ${ }^{65}$ These theories investigate multi-phonon non-radiative relaxation for both atomic and molecular types of traps in an ideal fcc lattice. The exciton wavefunctions and the lattice deformations are approximated as being exponentially damped, each with a different decay length, and the lattice vibrations are harmonic. Adiabatic energy surfaces for the trapping process were determined as a function of the inverse distortion length and the amplitude of the distortion. For the lighter rare gases, helium and argon, two types of traps were found: an atomic trap in which the lattice pulls away from the excited atom on which the exciton is localizing, thus forming a bubble around it, and (2) a molecular trap where two atoms move close together forming a bond similar to the excimer in the gas phase and where the remaining lattice sites do not move significantly at all. For krypton and xenon only the molecular traps are found, as the atomic traps are too shallow in energy to be populated. The study also revealed that the energy released in lattice relaxation, that is the depth of 
the trap, decreases with increasing atomic weight, and that when both trap sites are possible, it is easier to form the molecular one than the atomic one. This latter aspect is understandable in light of the fact that to form the atomic trap twelve atoms must move, whereas to form the molecular trap only two atoms must move.

The most important feature of these potential surfaces is the barrier separating the free exciton state from the trapped exciton state. It is crossing the barrier that determines the trapping rate at low temperatures. This process has been estimated to occur on the nanosecond time scale. Once the barrier has been crossed, the remainder of the trapping process takes place on a much faster picosecond time scale, probably via a mechanism much like that proposed by Martin. The trapping process involves either tunneling through the barrier or going over it. The optimal tunneling path is a compromise between the radius of the lattice distortion, that is how many sites must move off their equilibrium position, and the amplitude of the actual distortion made by each site.

To calculate the tunneling rates for their potential energy surfaces, Kmiecik and schreiber adapted a theory of multi-phonon non-radiative transitions by Huang and Rhys, 66 which assumed that the degree of localization of the exciton is not changing during the tunneling. The calculation was performed along a single interaction mode; that is the radius of the distortion in the lattice sites is fixed. This 
distortion parameter ( $\alpha$ in their paper) was treated as a variational parameter to optimize the tunneling rates. The results show that tunneling rates increase very rapidly as the distortion radius decreases, even though the barrier height increases as this width decreases. The distortion radius is such that second nearest neighbors are essentially not affected. Kmiecik and Schreiber also found that as the atomic weight increases, the barrier height and the amplitude of the distortion also increase. Lastly, they found that the size of the excitonic wavefunction is strongly localized: there was an amplitude of only $30 \%$ on neighboring sites for the molecular trap and less than $15 \%$ for the atomic trap. For the optimal tunneling rate in xenon, the barrier height was 44.20 mev resulting in a rate of $4.2 \times 10^{5} \mathrm{~s}^{-1}$. The rate of tunneling at the minimum in the barrier, $29.4 \mathrm{mev}$, was markedly lower at $2.4 \times 10^{-5} \mathrm{~s}^{-1}$.

These rates are much less than the rates proposed by Martin for his resonantly enhanced energy transfer trapping mechanism. However, the results are quite dependent on the experimental values used for the width of the exciton band and for the lattice relaxation energy. The difference of these two parameters is the experimentally measured stokes shift. A $10 \%$ decrease in the band width results in a factor of 10 increase in the tunneling rate. In fact, if a value of 0.35 meV for the bandwidth is used in the example of xenon (instead of $0.45 \mathrm{meV}$ used for the results quoted above), the resulting 
optimal rate is $1.27 \times 10^{9} \mathrm{~s}^{-1}$ at a barrier height of $30.4 \mathrm{meV}$.

In summary, self-trapped excitons form either from trapping of a free exciton or from recombination of a conduction band electron with a previously trapped hole. The trapped exciton in xenon is molecular in nature, strongly resembling the excimer in the gas phase. The state is Rydberg-like and only weakly perturbed by the adjacent atoms in the bulk. The site of trapping in the solid is either a vacancy defect or two atoms in the bulk crystal, depending on temperature, and it is a defect-like site in the liquid. Luminescence from the free exciton state is at $8.35 \mathrm{eV}$, very close in energy to the atomic absorption line, while luminescence from the vacancy trapped exciton state is at $7.2 \mathrm{eV}$, and that from the bulk trapped exciton is at $7.6 \mathrm{eV}$. In the liquid or supercritical fluid there is a threshold density at which excitons appear in the spectrum. At this density clusters are assumed to exist that are large enough to support the exciton. In the trapping process at cold temperatures, there is a free exciton well and a self trapped well separated by a barrier of approximately $35 \mathrm{meV}$. The free exciton tunnels through this barrier on the 10's of nanoseconds time scale at cryogenic temperatures, but faster with increasing temperature due to increased population near the top of the barrier. Once through the barrier, the exciton traps quickly on a time scale of a few picoseconds via a resonant hopping mechanism. 


\subsection{Time Resolved Measurements:}

2.5.1 Luminescence Experiments: A number of experiments in the time domain have been carried out, all on the nanosecond time scale. The excitation sources are either pulsed electron beams or synchrotron radiation. Nearly all of these studies reveal that the broad emission of the trapped exciton at either 7.2 or $7.6 \mathrm{eV}$ contains two components: one with a lifetime of approximately 25 nanoseconds, corresponding to the radiative decay of the triplet molecular self-trapped exciton, and the other with a faster decay of approximately 5 nanoseconds, corresponding to the singlet self-trapped exciton. A few studies have also been done on the free exciton decay and the radiative recombination of trapped holes and electrons.

The self-trapped excitons are considered primarily as excimers solvated by the rest of the solid or liquid in an only slightly perturbative manner, as has been described above. As a point of reference, then, it is reasonable to expect the radiative lifetimes of the excitons to correspond to those of the related molecular states in the gas phase. Experimental measurements ${ }^{67,68}$ of the lifetime of the lowest two electronic states of the excimer are $6 \mathrm{~ns}$ for the singlet state, denoted by either $o_{u}^{+}$or ${ }^{1} \Sigma_{u}$, and $100 \mathrm{~ns}$ for the triplet state, denoted by either $1_{u}$ or ${ }^{3} \Sigma_{u}$. The decay of the triplet states is thought to depend on the degree of mixing with the $\pi_{u}$ states by the spin-orbit interaction. This mixing is 
greater under the influence of the crystal field than in the gas phase, hence the triplet state of the trapped exciton is shorter lived than that of the gas phase excimer.

Monahan et al. excited solid xenon at $80 \mathrm{~K}$ with synchrotron radiation resonant with the $n=1$ and 2 states of the $\Gamma(3 / 2)$ series and the $n^{\prime}=1$ state of the $\Gamma(1 / 2)$ series. ${ }^{69}$ They collected the resulting emission in the wavelength region between 110 and $190 \mathrm{~nm}$ without the use of a monochromator. The luminescence decay with the excitation resonant with the $\mathrm{n}=1$ state was instrument limited with a 5 ns rise and fall. It is not clear why the more typical decay time scale of $25 \mathrm{~ns}$ was not seen. The luminescence with excitation at the energy of the $\mathrm{n}=2$ peak contained several components, the dominant one being a 58 ns decay. The authors assigned this as a decay of the $n=2$ state. They further suggested that since at 80 $\mathrm{K}$ the luminescence is primarily observed at $7.6 \mathrm{eV}$, that the $7.6 \mathrm{eV}$ radiation is from the $\mathrm{n}=2$ state, while the 7.1 to 7.2 $\mathrm{eV}$ radiation is from the $\mathrm{n}=1$ state. This is not consistent with the interpretation of both frequencies corresponding to emission from the $n=1$ state, but at different lattice sites as suggested by Kink et al. Lastly, excitation resonant with the $n^{\prime}=1$ state also produced an instrument limited rise and decay, but with a shoulder in the decay, which the authors attributed to an unidentified energy exchange process.

Kubota et al. examined the similarity between the trapped excitons and gas phase excimers on the basis of lifetimes of 
the two lowest electronic states of the dimer, the triplet and the singlet, in the presence of an applied electric field (as described above for their experiment on the relationship between free carriers and excitons). ${ }^{70}$ Argon, krypton, and xenon were excited with ${ }^{207} \mathrm{Bi}$ decay, and all three luminesced on both a fast and slow time scale. The fast component time constant was 2 to 5 nanoseconds, on the order of the instrument response time, while the slow component time constant decreased with increasing atomic weight: Ar: $860 \mathrm{~ns}, \mathrm{Kr}: 80$ ns, Xe: $27 \mathrm{~ns}$, in keeping with the increasing spin-orbit coupling in the series. The fast decay times are consistent with the times reported for the rare gas excimers in the gas phase and so support the notion that the exciton is only weakly perturbed by the surrounding bulk, while the long decay is significantly faster than in the gas phase. It is also noteworthy that the long decay times for all three gases decreased with the application of the field, suggesting that charge recombination and the subsequent relaxation to the lowest exciton state is not significantly faster than the radiative lifetime of the relaxed exciton, and in fact may be slightly slower.

For xenon the ratio of integrated intensity of the triplet state to that of the singlet state is $20: 1$. The ionization source either preferentially excites states that decay to the triplet state, or the singlet states are converted to the triplet states before luminescence can occur, possibly due 
to the large spin-orbit coupling.

Keto et al. have done a temperature study of the luminescence decay times for excitons in solid and liquid argon and xenon that indicates that the solid and liquid phases have similar radiative lifetimes. ${ }^{71}$ The excitation source was a $200 \mathrm{keV}$ electron beam. They found the decay times to be temperature independent from 80 to $130 \mathrm{~K}$. In solid xenon the $n=1$ and $n=2$ states of the $\Gamma(3 / 2)$ series are both found to be present over this temperature range. Following $z$ immerer et al., ${ }^{72}$ Kubota et al. assigned the $7.6 \mathrm{eV}$ band to the $\mathrm{n}=2$ state and the $7.1 \mathrm{eV}$ band to the $\mathrm{n}=1$ state, in contrast to the two site interpretation used by other authors. Single exponential fits to the data resulted in rise times of 3 ns and decay constants of $28 \mathrm{~ns}$ and $16 \mathrm{~ns}$ respectively for the $\mathrm{n}$ $=1$ and $\mathrm{n}=2$ states in the solid and a $32 \mathrm{~ns}$ decay constant for the liquid (average value for 4.5 to 15 atmospheres and temperatures ranging from the melting to boiling points). There was no observation of the shorter component seen by Kubota, and the authors suggested that the recombination of the trapped holes (ions) and electrons is strongly preferential to forming the triplet state.

Keto et al.'s previously published value for the collisionally induced conversion of the triplet state to the singlet state ${ }^{67}$ indicates that this process in conjunction with a radiative decay should result in an experimentally observable decay time of $93 \mathrm{~ns}$ for the triplet state, far longer 
than what is observed in the condensed phase. However, the large number of electrons impinging upon the sample in these condensed phase experiments provided another collision partner for the excited gas atoms until the electrons recombine or react and disappear. The collisions of electrons of this energy regime and rare gas atoms are nearly elastic; consequently the electrons thermalize slowly. Using a Langevin model for the electron motion, the authors estimated that within 11 ns the Electron density will have decreased due to recombination to a point too small to interact significantly with the excitons. This suggests that electron recombination is relatively fast in the condensed phase, and the authors surmise that since the 7.1 and $7.6 \mathrm{eV}(\mathrm{n}=1$ and $\mathrm{n}=2$ in their interpretation) states decay at different rates, the electron recombination must occur before they luminesce. Otherwise the luminescence would be at the same rate for both energies as a result of mixing with the singlet state. This further implie: that the recombination must be faster than the 3 ns risetime of these two states. This is in contrast to the work of Kubota et al. just mentioned above that suggested that the electron recombination time was approximately equal to or even longer than the radiative lifetime of the exciton.

Luminescence experiments by Hanus et al. reveal that at low temperatures both the free and trapped excitons could exist simultaneously in solid xenon. ${ }^{73}$ The authors suggested that since the fast trapping rate $\left(10^{12} / \mathrm{sec}\right)$ predicted by 
Martin is much greater than the relatively long nanosecond radiative lifetime of the trapped state, the emission intensity from the trapped state should be much greater the emission from the free state, and this was seen experimentally.

Starting in the 1970's zimmerer et al. conducted several experiments devoted to determining the temperature dependence of the exciton luminescence as well as the rate of decay of the free excitons into the self-trapped excitons. Like Hanus, they also noted the intensity difference in the luminescence from the free versus the trapped excitons in solid xenon, but suggested that the high energy electrons used as an excitation source by Hanus et al. may have vaporized their sample, and the resulting gas phase atoms may have been the source of the narrow lines that were assigned to the free excitons. ${ }^{74}$ In their experiment $\mathrm{zimmerer}$ et al. used a much weaker electron source, but they also saw emission from free excitons at $\mathbf{8 . 2}$ $\mathrm{eV}$ and from trapped excitons at $7.2 \mathrm{eV}$. The ratio of intensity of free exciton emission to that from trapped excitons is 1:500. They also noted that on:y well annealed samples produced the free exciton emission and suggested that defects may lower the barrier between the free and trapped state wells, thus allowing faster trapping. The authors equated the ratio of the emission intensities from the free and trapped exitons with the ratio of the rates for the two paths of free exciton state decay--emission and trapping. 
Using the gas phase lifetime as the inverse of the free exciton radiative rate, they calculated the trapping rate from the intensity ratio to be $5 \times 10^{10}$ to $10^{12}$, values a bit less than those predicted by Martin.

Later luminescence experiments on solid xenon between 4 $\mathrm{K}$ and $30 \mathrm{~K}$ by $\mathrm{zimmerer's}$ group $^{75}$ complement those described above by keto et al. In contrast to the moderate temperature results of Keto's group, zimmerer et al. found a striking temperature dependence in the long decay component at these low temperatures: the decay time varies from $900 \mathrm{~ns}$ at $4 \mathrm{~K}$ to only $150 \mathrm{~ns}$ at $30 \mathrm{~K}$. A faster component with a decay time of 3 ns was in full agreement with Kubota's work. The authors used a model for phonon-assisted depopulation of the triplet state into the singlet state to explain this temperature dependence. They distinguished this from a phonon activated quenching of the triplet state to some non-radiative state since the intensity of the emission did not change with temperature. (Note: the triplet and singlet are very close in energy and both emit at $7.2 \mathrm{eV}$ ). The authors acknowledged, however, that while the fitting procedure gave consistent Iifetimes for the triplet and singlet states, the rates for crossing to and from the two states contained large error bars. Nonetheless the rates were on the order of 10 to 100 nanoseconds.

Zimmerer et al. closed this paper with an alternative explanation of the temperature dependence in which the triplet 
state is split into its constituent states by the crystal field, which are separated in energy on the order phonon energies. As the temperature is changed, the relative population of these states change. Since they have different oscillator strengths for transitions to the ground state, the observed decay times change with temperature. Keto et al. countered that since zimmerer's experiment utilized low energy electrons for the excitation of the xenon, zimmerer et al. may have inadvertently excited a large population of surface states, which might therefore require reevaluation of their results.

The temperature dependent studies by $\mathrm{Z}$ immerer et al. also encompassed the free exciton region near $8.3 \mathrm{eV}$ of the Iuminescence spectrum, the dynamics of which is indicative of the tunneling rate into the trapped state. ${ }^{76}$ The selftrapping rate was calculated using gas phase values for the radiative contribution to the experimental decay and found to increase from slightly less than $2 \times 10 \%$ second at $10 \mathrm{~K}$ to over $10^{10} /$ second at $40 \mathrm{~K}$. The ratio of intensities between tht free and trapped excitons did not change nearly so much within this range of temperatures. This means that zimmerer et al.'s earlier equating of the ratio of intensities with the ratio of rates for radiative decay versus trapping decay for the free excitons was incorrect. In fact, the intensity ratio remained smaller than the rate ratio over this entire temperature range. The trapped exciton luminescence was too great to be 
accounted for by only the apparent free exciton population available to tunnel into the trapped state. The implication of this result was that there was another source of trapped excitons besides the free exciton population giving rise to luminescence at $8.4 \mathrm{eV}$. This was further supported by the fact that the trapped luminescence rose immediately, and did not correspond in time to the decay of the free exciton emission.

An explanation for this difference in the decay of the free state population and the rise of the trapped state population lies in their excitation wavelength. In order to avoid scattered light at the energy of the free exciton, $z$ immerer et al. set the excite wavelength at the $n=2$ exciton energy. The authors proposed that the result was a production of hot excitons, some of which immediately surmounted the barrier between the free and trap states, became trapped and then cooled, while others thermalized in the free well via phonon collisions and remained free for a time before tunneling through the barrier and trapping. Thus, it is only the thermalized free excitons that exhibit the observed temperature dependence in the self trapping rate; the hot population crosses into the trapped state before luminescence can occur at the free exciton energy. The intensity of the free exciton luminesence is governed by thermalization rate of the free excitons. If this rate is faster than the the tunneling rate, more luminesence from the free state will 
occur. The free versus trapped state luminescence intensity ratio should thus be equated with a ratio of the phonon scattering rate for the hot excitons, an estimate of the cooling rate, to the Debye frequency, which is an upper estimate of the tunneling rate, and in fact this ratio and the intensity ratio are both on the order of 0.01 to 0.001 . The temperature data were found to fit Rashba's theory of thermal assisted tunneling, which predicts a trapping rate of the form $\Gamma(T)=\Gamma(0)(1-T / \theta)^{-\alpha}$ for $T<<\theta$, the Debye temperature. $\pi$ Lastly, the authors calculated a barrier height of $25 \mathrm{mev}$, in accord with the value of $20 \mathrm{mev}$ calculated by Fugol'20 using Stokes shift data, but smaller than the value of $35 \mathrm{mev}$ calculated by Kmiecik and Schreiber.

The most recent work by $z$ immerer et al. on the trapping rate problem indicates that the rates described in the last paragraph depend strongly on defect concentrations in the crystals. ${ }^{78,79}$ By growing very pure crystals, they increased the free exciton luminescence intensity from what had been previously observed. They noted that while the trapping rate for these crystals was only on the order of $10^{8} /$ second, it increased with increasing temperature, as had been seen in the defective crystals. This trapping rate is commensurate with the rates predicted by the theory mentioned above by Kmiecik and Schreiber. The authors concluded that while the experiments agree with the theory, the observed rates may still be too large and that the process is still far from understood. 
2.5.2 Transient absorption experiments: Much less work has been done using transient absorption techniques than has been accomplished in the study of the characteristics of the luminescence of the rare gas excitons. Most of what has been done has involved transient absorption studies of neon, argon, and krypton, and all of the work is on the nanosecond time scale and longer.

Kanzaki et al. have obtained transient absorption spectra of the rare gases excited with a $5 \mathrm{~ns}$ pulse of $500 \mathrm{keV}$ electrons. ${ }^{80,81}$ They probed with either a tungsten or xenon arc lamp and found that solid argon, krypton, and xenon all have transient spectra that contain a peak near $1 \mathrm{eV}$ and very broad tail that extends to the blue. similar to the luminescence data, the absorption decay times decreased with increasing atomic weight: argon: $1.41 \mu \mathrm{sec}$, krypton: 120 ns, and xenon: 30 ns. For solid neon, argon, and krypton the decay constants for the luminescence and transient absorption were equal. The transient absorption decays were found to be the same at all wavelengths. This implies that the relaxation to the bottom of the molecular self-trapped exciton well is faster than their instrument response of $10 \mathrm{~ns}$. The absorption data were interpreted as transitions from the lowest exciton state, the triplet state, to the anti-bonding orbitals that correlate to the same atomic ns states as the triplet state and to the next higher manifold of states that correlate 
to the atomic np states. More specifically, the red end of the spectru.n is assigned to the ${ }^{3} \Sigma_{u} \rightarrow{ }^{3} \Sigma_{g}$ and ${ }^{3} \Sigma_{u} \rightarrow{ }^{3} \pi_{g}$ transitions of the Rydberg electron, and the higher energy transition is assigned to the ${ }^{3} \Sigma_{u} \rightarrow{ }^{3} \Sigma_{g}$ transition corresponding to the ${ }^{2} \Sigma_{u} \rightarrow{ }^{2} \Sigma_{g}$ hole transition in the $R_{2}^{+}$core. This latter transition is very broad due to the steep repulsive wall of the final state. This same scheme of low energy transitions, arising from the outer Rydberg electron and high energy ones from the atomic cores has been used to interpret the absorption spectrum of the alkali halides. ${ }^{82}$

Kanzaki et al. observed shifts in the energy of the transient absorptions between the three phases for all the rare gases, which they explained in terms of the electron affinity of these elements. In neon and argon, the transitions were found to be shifted to higher energies in going from solid to liquid to gas, while in krypton and xenon the gas transitions were found higher in energy. The final state is more delocalized than the triplet exciton state, and as such is shifted up in energy in neon and argon since they have negative electron affinity, while in krypton and xenon it is decreased in energy because of the positive electron affinity.

Schwentner et al. have also conducted transient absorption measurements on the rare gases using a pulsed electron beam as an excitation source and an arc lamp for the probe. ${ }^{83}$ The same transition assignments were made as in Kanzaki's work. The authors noted that the lack of a vibrational 
progression indicates that the 0-0 transition must be favored by the Franck-Condon factors and that this is a notable characteristic in a Rydberg type transition. They also suggested that the blue tail contains transitions to the conduction band that are more important than those within the ion core. As a check of the lifetimes, they conducted luminescence experiments and obtained decays on the scale of a few nanoseconds from the singlet state and longer decays ranging from 0.1 to $1 \mu$ sec, presumably from the triplet state. These times are longer than previously reported, but the authors indicated that the differences may be due to their high excitation density. The main xenon exciton absorption band was too far towards the IR region for them to record it fully.

In discussing their data for argon and krypton, Schwentner et al. have depicted the energy level scheme of the exciton in a novel way. They suggested two approaches to understand the situation: either think of an excited atom forming an excimer with a gas phase partner and then inserting this excimer into the bulk crystal, or think of putting an excited atom into the crystal to form a free exciton, which then traps itself via relaxation of the surrounding atoms. The end result is the exciton state lying below the atomic states and a compression of the rest of the states into the region above this due to the decrease in the ionization energy of the bulk as compared to the free atom. 
This vast body of work that has so far been completed in an effort to understand the behavior of excitons in rare gases has opened several questions, some of which I address in my transient absorption experiments on supercritical and liquid xenon near room temperature (explained in detail below in Chapter 4). To date no one has directly measured the cooling rate of the excitons in the trapped state well to test the theory of Martin. This requires picosecond resolution, and thus far all the time resolved experiments have been on the nanosecond time scale. If the trapping process forms a molecular moiety similar to the gas phase excimer, as has been generally accepted, then the relaxation within the trapped state well should exhibit a shift in the absorption spectrum as the "molecule" vibrationally relaxes. This will manifest itself in a wavelength dependence of the transient absorption decay: The spectrum will shift to the blue with time.

Tunneling times in the liquid phase have also not been measured directly. Experiments done so far have been on crystaliine samples. The presence of defects has been shown to facilitate the tunneling process through the barrier separating the free and trapped excimer states. In the liquid, which is "defective" by nature, this process should be very fast, most likely also on the picosecond timescale since defective solids had tunneling rates estimated to be in the hundreds of picoseconds.

steinberger's density experiments suggested that in the 
liquid both gas-like excimer and solid-like excitons exist simultaneously. His results indicate that there exists a critical density at which the liquid density fluctuations are able to form clusters large enough to support an exciton. This should be reflected in the dynamics of the transient absorption signal. Below this critical density the relaxation rate should scale with density since collisions with ground state atoms is the primary means of energy relaxation of the excimer. Above this critical density the resonant hopping mechanism described by Martin is the dominant relaxation mechanism. It is not clear what the density dependence of this mechanism should be. It is within a momentary cluster of atoms within the liquid that the exciton exists, and it is not clear how the density of this cluster is influenced by the bulk liquid density. It is possible that temperature is the more important variable in this case, as it is temperature that describes the average velocity of the two atoms in a collision, and thus the ability of a ground state atom to get close enough to an atom in the excimer molecule to effect the resonant energy transfer. 


\section{Chapter 3}

\section{Experimental Details}

\subsection{Laser system And optics common to Transient Absorption}

and Fluorescence Experiments: The laser system used for these experiments has been described in detail elsewhere, so only a brief description will be provided here.84,85,86 The heart of the system was a synchronously pumped dye laser which was amplified with a Q-switched Nd:YAG, as shown in Figure 7. The dye laser was a Coherent model CR-599 with an extended cavity using rhodamine R6G in ethylene glycol for the lasing medium. A Spectra Physics model 2030 argon ion laser modelocked at $514.5 \mathrm{~nm}$ was used to pump the dye laser. The ion laser output was $400 \mathrm{~mW}$ with 110 ps pulses at a repetition rate of 80.6 MHz. The dye laser output was $24 \mathrm{~mW}$ at a wavelength of $590 \mathrm{~nm}$ with pulses 2 ps long, also at $80.6 \mathrm{MHz}$. The dye laser output was amplified using three flowing Kiton Red dye cells, each of which was longitudinally pumped with the Nd:YAG, a Molectron MY-34. The Nd:YAG $\operatorname{ran}$ at $10 \mathrm{~Hz}$ and delivered $250 \mathrm{~mJ}$ pulses at $532 \mathrm{~nm}$. The timing of the amplification was controlled with homemade circuits utilizing the rf output of the modelocker referenced to the Q-switch of the Nd:YAG as a timing source. The final output of the amplifier chain was 2 ps pulses at a $10 \mathrm{~Hz}$ repetition rate, each with an energy of $1 \mathrm{~mJ}$ and 
Figure 7. This is a schematic of the $10 \mathrm{~Hz}$ amplified system and a partial list of the optics currently in use in this system. The beam continues from these optics into those for the experiment, shown in Figure 8. For a more complete description, consult the theses of Mark Berg and Alex Harris. Note that occasionally an optic will be changed in routine maintenance and this list may not remain up to date. The numbers below refer to the numbers adjacent to the optics in the figrire.

1: Molectron MY-34 Q-switched Nd:YAG laser.

2: Spectra Physics 2030 modelocked argon ion laser.

3: Coherent CR-599 dye laser using rhodamine R6G.

4: Newport broadband mirror, one inch, BD.1 coating.

5: CVI BSR-5300-1 dichroic, $R>99.5 \%$ at $530 \mathrm{~nm}, \mathrm{~T}>85 \%$ at $1064 \mathrm{~nm}, 45^{\circ} \mathrm{s}$-pol., AR coating on back surface.

6: Melles Griot 02WBK003/078, beamsplitter, $\mathrm{R}=10 \%$, $\mathrm{T}=90 \%$.

7: Melles Griot 01LPX177, $10 \mathrm{~cm}$ plano-convex lens.

8: Melles Griot 01LPK003/078, $-4 \mathrm{~cm}$ plano-concave lens.

9: CVI beamsplitter, $R=60 \%, T=40 \%$, AR coating on back surface.

10: long focal length lens.

11: Dichroic, 1 inch diameter, $3 / 8$ inch thick, $45^{\circ} \mathrm{s}-$ pol, $R>95 \%$ at $532 \mathrm{~nm}, T \max$ at $583 \mathrm{~nm}$.

12: Kiton Red flowing dye cell.

13: Dichroic, 1 inch diameter, $3 / 8$ inch thick, $22.5^{\circ} \mathrm{P}-$ pol, $R>99.5 \%$ at $532 \mathrm{~nm}, \mathrm{~T} \max$ at $582 \mathrm{~nm}$.

14: $2.5 \mathrm{~cm}$ symmetric convex lens.

15: Crystal violet saturable absorber jet.

16: Melles Griot LDX103/078, $5 \mathrm{~cm}$ symmetric convex lens.

17: Melles Griot 532 high reflector, HEBAR coating, $45^{\circ}$ S-pol.

18: $2 \mathrm{~cm}$ plano-convex lens.

19: $8 \mathrm{~cm}$ plano-convex lens.

20: CVI dichroic, 2 inches diameter, $45^{\circ} \mathrm{P}-$ pol, $R>95 \%$ at $532 \mathrm{~nm}, \mathrm{~T}>75 \%$ at $580 \mathrm{~nm}$.

21: CVI dichroic, 2 inches diameter, $45^{\circ} \mathrm{s}-\mathrm{pol}, \mathrm{R}>95 \%$ at $532 \mathrm{~nm}, \mathrm{~T}>65 \%$ at $580 \mathrm{~nm}$. 


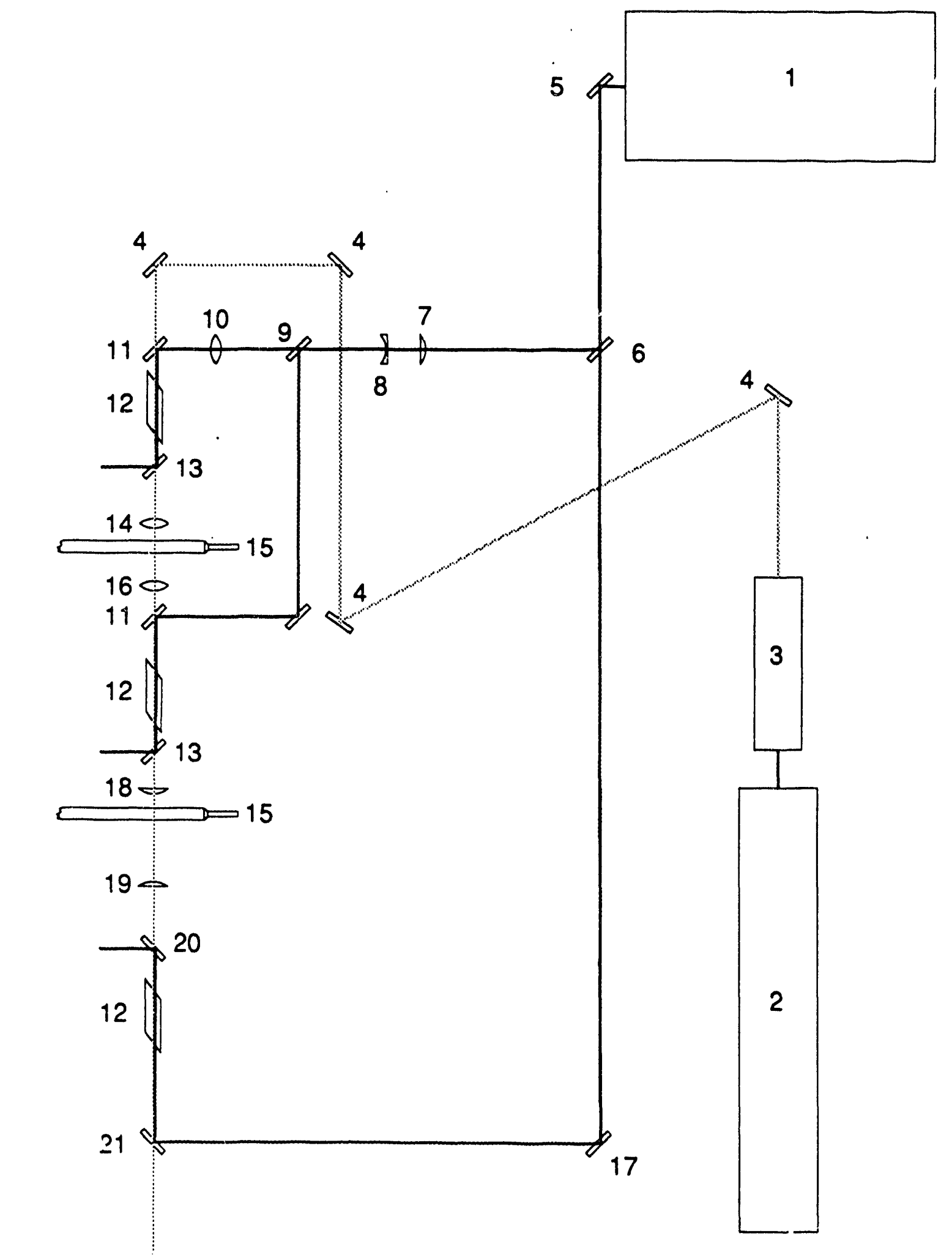

Figure 7: Synchronously pumped ps dye laser and amplifier 
wavelength of $590 \mathrm{~nm}$. Shot to shot fluctuations were on the order of 10 percent.

The amplifier output was directed to the experimental optics set-up shown in Figure 8 . The amplified beam was frequency doubled using a KDP crystal, yielding 2 ps pulses at $295 \mathrm{~nm}$ with $100 \mu \mathrm{J}$ apiece. This was more than adequate UV power for these experiments, and in fact the UV beam had to be attenuated to prevent generation of signal from the sample cell windows via multiphoton excitation. The UV beam was used as the pump beam in both the transient absorption experiments and the fluorescence experiments on the xenon. For the transient absorption studies, the UV beam was delayed relative to the probe beam using an optical delay line consisting of a quartz corner cube mounted on a computer controlled Klinger one meter translation stage. The double pass configuration of the UV beam on the stage coupled with the $10 \mu \mathrm{m}$ stage steps resulted in a small step size resolution of 67 fs (or 15 steps per ps).

3.2 Transient Absorption Experiments: The probe beam was a portion of a white light continuum generated in a $10 \mathrm{~cm}$ liquid cell using the residual $590 \mathrm{~nm}$ light left after the second harmonic generation. Water was used in the cell for all wavelengths except $710 \mathrm{~nm}$ and $900 \mathrm{~nm}$, for which acetone was used. These two wavelengths correspond to the first and second Raman lines respectively in the continuum generated in 
Figure 8. This is a list of the optics used in the actual experimental portion of the setup. The beam enters this setup immediately upon leaving the amplifier chain. The numbers below refer to those adjacent to the optics in the figure.

1: Newport KPX199AR.14, $20 \mathrm{~cm}$ plano-convex glass lens, coating for $420-700 \mathrm{~nm}$.

2: Newport KPCO4OAR.14, $-5 \mathrm{~cm}$ plano-concave glass lens, coating for 420-700nm

3: KDP doubling crystal.

4: Newport SPC034AR.10, $-5 \mathrm{~cm}$ plano-concave fused silica lens, coating for 250-420 nm.

5: Newport SPX049AR.10, $20 \mathrm{~cm}$ plano-convex fused silica lens, coating for 250-420 $\mathrm{nm}$.

6: CVI dichroic, $45^{\circ} \mathrm{S}-\mathrm{pol} \mathrm{T}>80 \%$ at $590 \mathrm{~nm}, 45^{\circ} \mathrm{P}$-pol $R>998$ at $295 \mathrm{~nm}$.

7: CVI UV mirror, $45^{\circ}, T$ max at $295 \mathrm{~nm}$.

8: Precision optical UV quartz corner cube, 2 inch diameter, 5 arcsecond parallelism, no coatings, mounted on one meter Klinger translation stage with $10 \mathrm{\mu m}$ steps.

9: Newport broadband mirror, BD-1 coating.

10: Melies Griot 01LPX123/078, $10 \mathrm{~cm}$ plano-convex lens.

11: Quartz continuum cell, $5 \mathrm{~cm}$ long, filled with either water or acetone.

12: Dittrich bandpass filter, $10 \mathrm{~nm}$ bandwidth at $50 \% \mathrm{~T}$, $400 \mathrm{~nm}$ - $1000 \mathrm{~nm}$ range.

13: Neutral Density filters to bring continuum light within linear regime on $A / D$.

14: $15 \mathrm{~cm}$ plano-convex lens.

15: 600 micron spatial filter.

16: Melles Griot 01LQF078, $10 \mathrm{~cm}$ fused silica planoconvex lens.

17: Melles Griot 02MPG007/028, aluminum mirror.

18: uncoated optical flats.

19: $10 \mathrm{~cm}$ plano-convex fused silica lens.

20: Xenon cell.

21: $20 \mathrm{~cm}$ plano-convex fused silica lens.

22: microscope slide to block UV pump.

23: 20-25 cm glass plano-convex lens.

24: long wavelength cut-off filters, neutral density filters.

25: DT110 photodiodes.

26: Calibrated UV neutral density filters for controlling pump beam intensity. 


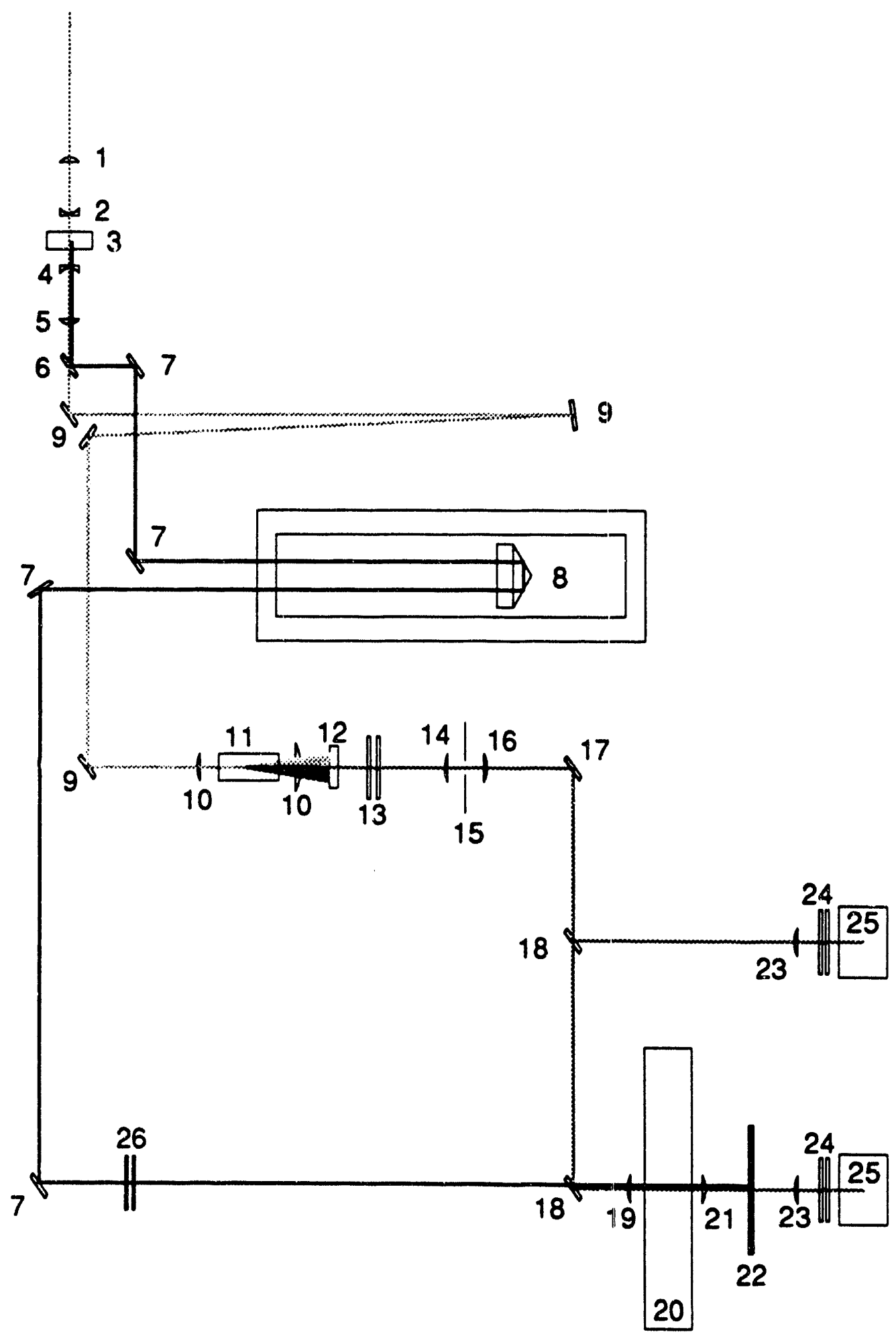

Figure 8: Optical setup for transient absorption experiments 85 
acetone, and as such had much more intensity than the water continuum. Wavelengths were selected using three cavity bandpass filters with a bandwidth of $10 \mathrm{~nm}$. The usable wavelength range of the continuum was from approximately 400 $\mathrm{nm}$ to $1000 \mathrm{~nm}$. Beyond $900 \mathrm{~nm}$ the sensitivity of the photodiode detectors drops sharply. After passing through the bandpass filter, the beam was spatially filtered with a $600 \mu \mathrm{m}$ pinhole to improve focussing. The beam then passed through two $1 / 2$ inch thick silica flats, each reflecting about four percent of the light due to refractive index mismatch; thus, creating two relatively well balanced beams, one for use as a reference and one for interrogation of the sample. Neutral density filters were used to keep the light intensities within the linear range of the $A / D$ converter and to accurately balance the intensities in the two arms for good normalization. Normalization to 0.2 percent was realizable routinely at most wavelengths for collection of 10 laser shots. Stray light and remaining pump light were removed with colored cutoff and long pass filters. The pump and probe beams were overlapped and directed in a collinear geometry into the sample with a $10 \mathrm{~cm}$ focussing lens. The difference in focal plane location of the pump and probe beams due to dispersion of their often widely disparate wavelengths was compensated for by adjusting the collimation of the probe beam after the spatial filter.

The intensity of two beams of the continuum was measured 
with EG\&G DT-110 photodiodes. For wavelengths at which the continuum intensity fell below what could be detected with the photodiodes, ENI 600L rf power amplifiers (24 Db amplification) were inserted between the diodes and the $A / D$. The output of the diodes was fed into a Lecroy 2249 SG 10 bit A/D converter, gated and cleared by pulses generated by a Lecroy 2323 programmable dual gate generator that was triggered with the Na: YAG Q-switch. The linear range of the $A / D$ was approximately 350 to 900 counts. The A/D output was recorded by a 386 personal computer running a data collection program written by Daniel Russell. ${ }^{100}$ The actual data collection subroutine used in this program was written by myself and is discussed in appendix 2 herein. The software controlled the number of shots taken per stage position as well the stage movement, which in turn dictated the length of the scan and the amount of time between data points in the scan. Data processing involved subtraction of backgrounds in each channel, summing individual data scans, taking the base 10 logarithm to convert to absorption units, and finally a baseline subtraction to arrive at the change in absorption as a function of the time delay between the pump and probe pulses.

The overlap of the of the pump and probe beam focuses was optimized using a saturated solution of diphenyl-dibutadiene (DPB) in hexane. This sample gave rise to a large signal, a greater tha.l 20 percent change in transmission at most of the 
wavelengths studied. Once the beams were aligned with the DPB, the empty xenon cell was put into place.

since the UV power was sufficient to generate signal in the cell windows, presumably from formation of $f$-centers, the power in the UV was attenuated using calibrated neutral density filters with the empty xenon cell in place so as to result in no discernable window signal for the number of laser shots to be taken in the experiments. This typically required the insertion of about ND $=1$ in the UV beam.

The cell was then filled with xenon to the desired pressure and cooled to the desired temperature. Typical data scans had a change in transmission of a few percent, or a change in absorbance of order $10^{-2}$. For most wavelengths the system normalization was 0.25 percent or better, and less than 400 laser shots per point resulted in adequate signal to noise for small error bars in model fits to the data.

3.3 Fluorescence Experiments: The same UV pump configuration was used for the fluorescence experiments as was described above for the transient absorption experiments. The pressure cell has a very small collection solid angle of only 0.05 steradians, and collection of photons must be done along the same axis as the UV pump beam. For these experiments the collection lens was a $10 \mathrm{~cm}$ plano-convex lens with a 1.5 inch diameter. This effectively captured all the photons that left the cell in the forward direction, within the approximation 
that these photons originated from a point source, which was most certainly not the case. The fluorescence was focussed onto the slits of a Jerrall-Ash single monochromator with an f-matching lens. The grating in the monochromator was blazed at $550 \mathrm{~nm}$ and had 1200 grooves $/ \mathrm{mm}$. The detector for the fluorescence was an intensified array OMA, a Princeton instruments model IRY-700S/RB, bolted directly onto the monochromator over the exit slit area. No exit slit was used. In this configuration $45 \mathrm{~nm}$ of the spectrum could be collected at one time, tunable with the monochromator and dispersed over 740 channels of the OMA. Wavelength calibration was accomplished with a neon arc lamp placed as far up the UV beam line as possible. The OMA has a quantum yield of about 9 percent through the visible region, but only about 1 percent or less at $800 \mathrm{~nm}$ and longer. The UV intensity was adjusted with neutral density filters so as to prevent fluorescence from the cell windows. A glass microscope slide was used to block the UV pump beam, and cutoff filters, such as a schott RG-675, were used to block the microscope slide fluorescence and stray light. A background scan was taken with the cell empty and subtracted from the data collected with the cell filled with xenon. Integration times were typically half an hour per scan.

3.4 High Pressure cell: The pressure cell was constructed to conduct experiments in liquid xenon near the 
critical point: $847 \mathrm{psi}$ and $16.6^{\circ} \mathrm{C}$. Pressures of this order of magnitude can be contained with simple o-ring seals, and temperatures near room temperature can be controlled with a circulator and a simple water jacket made of a copper tube coiled about the cell body. The cell was designed to eliminate as much as possible the introduction of impurities into the xenon gas. Once it is filled with xenon, the cell is independent of a pumping system and is transportable by hand. As shown in Figure 9, the system consists of a storage bottle for the xenon, a pressure gauge, and the optical cell in which the sample resides for experiments. The flow of xenon is controlled by three valves between the tank and the cell. A tee and a fourth valve are provided for the initial evacuation of the system and admission of the xenon. The entire system is encased on three sides with $3 / 8$ inch thick aluminum and on the fourth with $1 / 2$ inch thick Lexan (otherwise known as polycarbonate or bullet proof glass) for protection of the user in the event of a rupture.

\section{4 .1 ** SAFETY NOTICE FOR OSERS OF PRESSURE CELL * *}

In spite of the shielding over the cell and tubing system, which should be ample to stop any fragments in the event of a rupture (1/4 inch Lexan can stop a police 0.54 caliber round), users are cautioned that such a system is never completely foolproof. Shields should be in place during filling and use of the cell. It is advisable that the user 
Figure 9: Diagram of high pressure oil free xenon cell. All fittings unless otherwise specified are Cajon VCR $1 / 4$ inch stainless steel with nickel plated silver gaskets. valves are numbered for discussion of cell operation in the text.

1: Glass Dewar for liquid nitrogen trapping of xenon.

2: Whitey 316-L-50df-4-150-EP, 316 stainless cylinder for xenon storage, 5000 psi, electropolished interior. Male VCR $1 / 4$ inch to $1 / 4$ inch national pipe thread connector welded to bottle.

3: 316 stainless steel tubing, 0.028 inch wall thickness, rated to 5000 psi.

4: Nupro SS-DLV5I diaphragm valve, $1 / 4$ inch female VCR connectors. Rated to 3500 psi in forward direction, 2500 psi in backward direction.

5: 0-3000 psi oxygen service pressure gauge, oil free. $1 / 4$ inch female VCR to $1 / 4$ inch female National Pipe Thread connector welded to gauge.

6: Modified High Pressure Equipment nipple, 1/4 inch 316 stainless, $1 / 4$ inch VCR gland welded to one end after cutting off the $\mathrm{HF}-4$ connector threads and cone.

7: $50 \%$ ethylene glycol/water coolant mixture. $1 / 4$ inch copper tubing wound around entire cell and connected with tygon tubing to cooler/circulator. The copper windings are covered with closed cell foam insulation.

8: Xenon cell, shown in detail in next figure. 


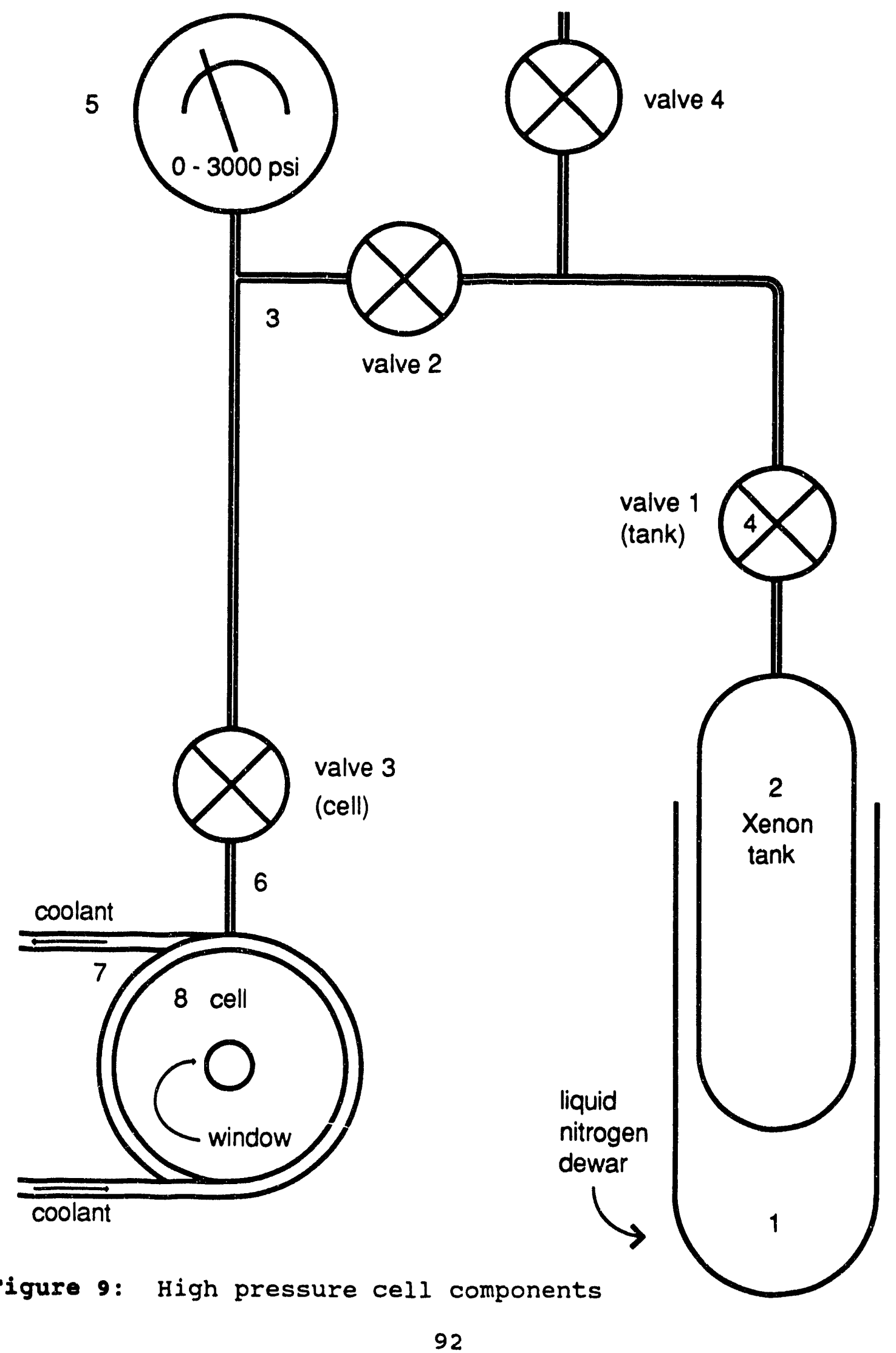


Figure 10: Interior details of high pressure cell. Inner bore is one inch in diameter. All parts electropolished or cleaned in hexane multiple times in an ultrasound cleaner.

1: Stainless steel endcap to hold other parts inside cell body under pressure.

2: Cell body made of 316 stainless.

3: Aluminum gasket, one inch diameter, $3 / 16$ inches thick.

4: Copper gaskets, one inch diameter, $1 / 16$ inches thick. One used with fused silica window, two used with sapphire window.

5: Janos, UV fused silica window, one inch diameter, 1/4 inch thick. Or Melles Griot 02WSA008, fused sapphire window, one inch diameter, $2 \mathrm{~mm}$ thick.

6: Teflon o-ring, one inch diameter, 1/8 inch thick.

7: Fitting for High Pressure Equipment HF-4 connector. 


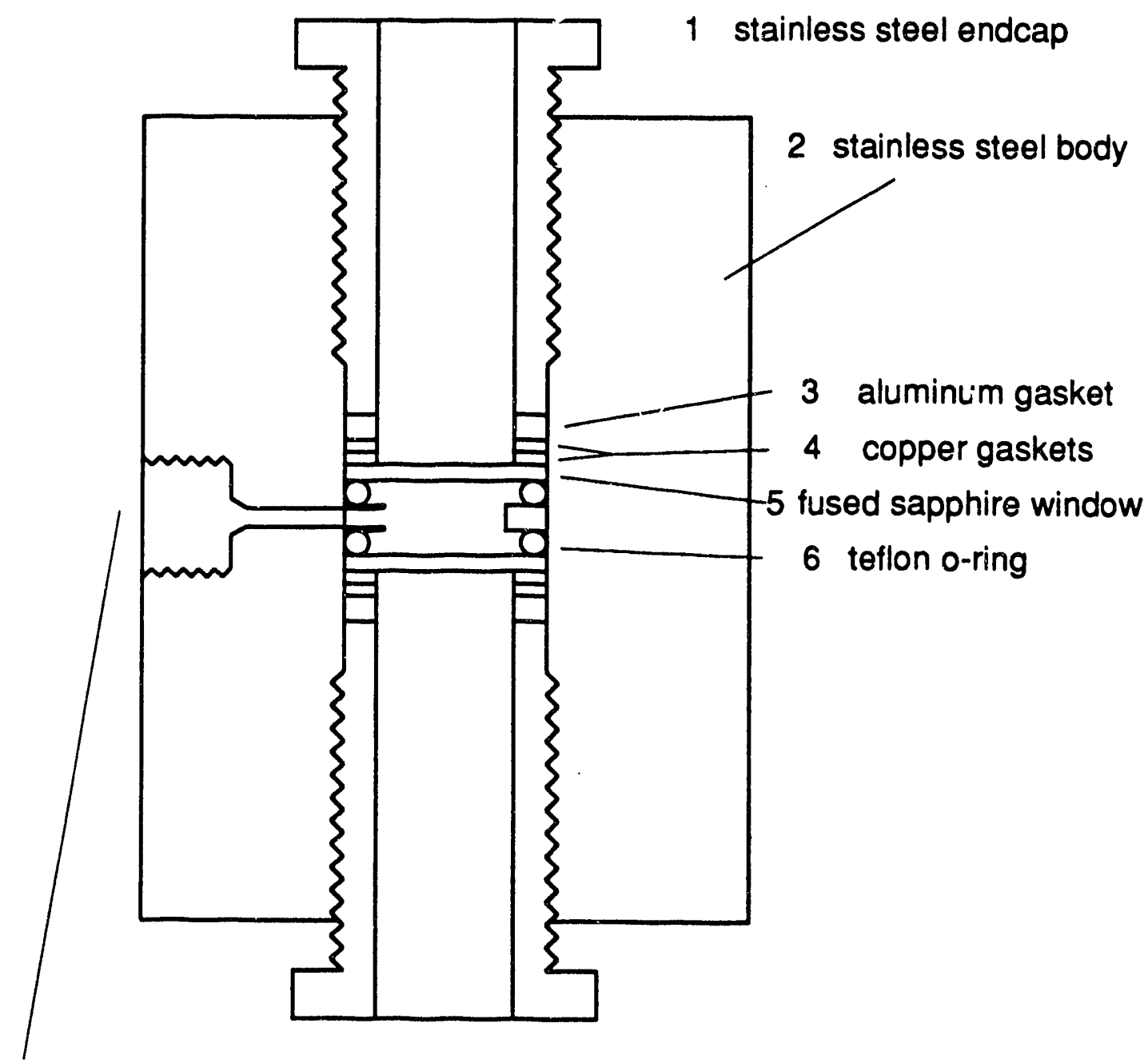

7 high pressure equipment HF4 fitting

Figure 10: Internal details of high pressure optical cell 
wear hearing protection, especially during filling and emptying of the optical cell, as the shock wave resulting from a rupture may be able to burst an eardrum, or at the very least cause a temporary hearing loss. Safety glasses are also advised. When the cell is under pressure it sholild be handled with care so as to minimize vibration or jolts. Never look into the windows of the cell, even if only a few psi of gas are in the cell. Use a mirror to look into the cell. Never tighten parts of the cell while they are pressurized. When filling the cell, stay in attendance and watch the pressure gauge while the tank warms (details below). The last third of the warming results in the greatest pressure increase, and there is no rupture disc or similar safety devise in this system for the safe diffusion of an over-pressure situation. This is because all of these devices have seals with lubrication in them, and as oils dissolve readily in liquid xenon use of such device would result in contamination. The weakest link in the system is the windows of the cell. They have the lowest pressure rating and must, unfortunately, be left uncovered to admit the laser beams used in the experiments. The user is advised to not stand in line with the optical axis when the cell is pressurized.

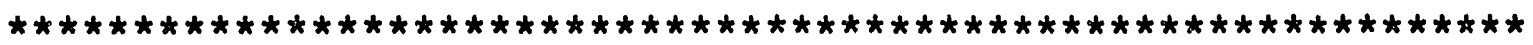
The following are the working pressure ratings of the cell components:

valves: forward (arrow direction): $3500 \mathrm{psi}$ 
backward: $2500 \mathrm{psi}$ ( the pressure beyond which the stem may stick closed in valve)

tubing: $5000 \mathrm{psi}$

gauge: damaged past 3000 psi

tank: $5000 \mathrm{psi}$

VCR connectors: that of the tubing

windows (empirically by Mark Paige): sapphire: 1500 psi quartz: $1200 \mathrm{psi}$

The pressure ratings of the windows may well be a little higher. We have never broken a sapphire window from pressurizing the cell with gases (though two broke in assembly from over tightening the endcaps), but a quartz window has been broken in this way. In addition, the quartz windows are more brittle than the sapphire ones, and pressurizing and emptying of the cell should be done slowly.

3.4.2 Filling the system with Xenon: Air and other gases present in the system must be first removed before xenon is introduced. This is most easily done with a tee arrangement with one arm of the tee connected to the system above valve number 4, one arm to the xenon source, and one arm to the pump.

In the prototype experiments evacuation of the cell was done using a diffusion pump equipped with a liquid nitrogen oil trap. This method is not recommended as the result was diffusion pump oil in the cell in spite of the trap (see 
discussion on impurity effects in appendix 1). Either xenon freezing on the inside of the trap pacified it and resulted in the passage of oils through the trap, or the trap was unable to prevent such passage regardless of the whether xenon was present in the system. An oil free pump, such as a turbo pump is recommended for the system evacuation. All valves should be open for the evacuation.

once the system is pumped to the $10^{-6}$ torr range, adequate for reducing the contamination levels to parts-per-billion, the xenon is admitted. The system should be flushed several times with xenon as an added precaution to expelling all of the air. Fill the dewar around the tank with liquid nitrogen (add as necessary to keep the dewar full during the filling of the tank) and open valve 4 to the xenon source. The filling time will have to be empirically determined, as it depends on the configuration used and the inlet pressure. As a point of reference, filling the system at a pressure of 5 psi for approximately 10 minutes resulted in about the right tank pressure. An indication that all the xenon in the lines has been trapped into the cold tank or that the tank is full is the cessation of boiling of the liquid nitrogen inside the dewar.

Close the cell valve (number 3 ) and valve number 4 . Remove the liquid nitrogen from the dewar by blowing nitrogen gas through the copper tube going into the dewar and allow the nitrogen gas to blow on the tank to speed warming. AGAIN, DO 
NOT CEASE TO WATCH THE GAUGE DURING THIS PROCESS AS OVERPRESSURE WILL DAMAGE COMPONENTS OR RESULT IN EXPLOSION. If the pressure exceeds approximately $1200 \mathrm{psi}$, bleed off some of the excess xenon through valve number 4 (disconnection of the pump and xenon source may be necessary). Taking the volume of the tubing between the cell and tank into account, filling the system to $1200 \mathrm{psi}$ with the cell closed off will result in a tank pressure of about $1500 \mathrm{psi}$ when all of the xenon is trapped into the tank for storage. $1500 \mathrm{psi}$ is adequate to reach experimental pressures, is a convenient starting point from which to fill the cell as one filling of the tube between valves 1 and 2 will result in a pressure in the optical cell of about 700 psi when the cell valve is opened, and provides some excess xenon should a small leak occur. Do not open valve 4 again at this point. Doing so will admit air into the system and necessitate pumping out the system and refilling.

\subsubsection{Filling the optical cell with Xenon: All valves} should be closed initially. CAUTION: IF THE TANK HAS BEEN FILLED TO 1500 PSI, OPENING ALL VALVES AT ONCE BETWEEN THE OPTICAL CELL AND THE TANK COULD BLOW THE WINDOWS OUT OF THE CELL. WEAR HEARING PROTECTION AND SAFETY GILASSES. KEEP BODY PARTS AWAY FROM THE WINDOWS. Open the tank valve slowly and close it again. Slowly open valve number 2 while watching the pressure gauge, and close it again. The valve sensitivity is adequate to control the pressure increase if the valve is 
opened slowly and to only a fraction of the full open position. The pressure should now be about 700 psi. slowly open the cell valve. The pressure will change very little. close all valves. Turn on the cooler and set it to $7^{\circ} \mathrm{C}$. To prevent water condensation on the windows from moisture in the air, hook the blower line to the house nitrogen and set the flow to a moderate level (noticeable against the back of one's hand). It is not necessary to have nitrogen blasting the windows to keep them clear. The cell will take about twenty minutes to cool. Open the cell valve again while looking into the cell WITH A MIRROR. Droplets of xenon squirting into the cell should be visible (putting a white card behind the cell on one side while shining a flashlight into the cell off the observation mirror will make the droplets appear dark and easy to see). Open valve 2 and let the cell fill watching the meniscus move out of the cell to insure that it does fill completely. To reach a pressuxe above that resulting at this point, close the cell valve and valve number 2 , and open AND CLOSE the tank valve. There should now be more than enough xenon in the tubing to fill the cell to at least 850 psi in the liquid phase. It is advised that valve number 2 and the cell valve be opened in an alternating fashion to avoid overpressurizing the cell. Once the desired pressure is reached, allow the cell to sit for fifteen minutes so the xenon added can fully coo?. Add more xenon if the pressure has dropped. All valves should be closed once the cell is 
filled to prevent loss of xenon in the event of a leak.

3.4.4 Trapping the Xenon out of the optical cell: Emptying the optical cell is much the same as the initial filling of the system as described above. Close all valves. Fill the dewar with liquid nitrogen and allow the tank to cool until the liquid nitrogen ceases to boil. Open and close the tank valve. Open valve number 2 while watching the gauge. If the pressure does not increase, the xenon pressure in the tank has been sufficiently reduced by the cooling so that all the valves between the tank and the optical cell can now be safely opened. This should be done slowly so as to prevent a sudden pressure drop that could damage the gauge or cause the windows to relax and crack. It is advisable to leave a small amount of pressure in the cell so as to keep the windows pushed snugly against the o-ring seals to prevent air from entering the cell during periods of disuse. Close the tank valve first to leave 20-30 psi in the system outside the tank. close the cell valve and valve 2. To prevent excessive ice build up and condensation in the dewar, blow the liquid nitrogen out of the dewar by flowing nitrogen gas through the copper pipe that hangs into the dewar bottom. If the nitrogen is left blowing on the dewar, the tank will warm up to full pressure within 2.5 hours. If the cell is going to be refilled at this point, it is not necessary to wait until full pressure is reached. Filling may be done at any time after the tank has reached the 
desired final pressure. If the nitrogen is not left blowing on the tank, the poor air circulation around the tank will result in a warming time in excess of eight hours to reach full pressure. Once the xenon has been removed from the cell, the circulator can be turned up to room temperature to warm the cell up so that the blowers can be turned off without the windows being covered with condensation.

3.4.5 Final Notes on cell construction: Several versions of the cell were actually constructed in an effort to maximize ease of modification and disassembly while still preserving an oil free environment within the system.

Fittings: The first version was assembled using swagelok connections, which are rated to a pressure equal to that of the tubing used. In this case the tubing had a rating of 5000 psi, but the Swagelok fittings were found to leak at less than $500 \mathrm{psi}$. Various amounts of tightening were employed to overcome this problem, but no effect was noticed with $3 / 4$ to over 2 turns of the nut (1.25 turns being specified as correct in the manufacturer's instructions). An additional problem with the swagelok fittings is the interlocking nature of their construction, which prohibits removal of a component without also unfastening adjacent components. Later versions employed Cajon VCR fittings with silver plated nickel gaskets. These fittings worked flawlessly with no leaking after assembly. An added feature of the VCR fittings is their zero clearance 
method of fastening, which allows removal of a part from the line by simply unscrewing the fitting nuts--there is no need to pull the tubing out of the fitting.

Valves: The cell was constructed with Nupro diaphragm valves (\#SS-DLV51), as these were the only available valves with the packing material and associated lubrication isolated from the wetted or flow area that had a sufficiently high pressure rating and that came stock with cajon VCR fittings. Stellite stem tips were used since they can be cleaned and do not absorb possible impurities. These valves come stock cleaned in trifluoroethane, and as such require no further cleaning to be used in the system.

mindows: The windows of the early cells were one inch diameter $2 \mathrm{~mm}$ thick fused sapphire (Melles Griot \#02WSA008), as this material is not prone to breakage from shock. Since sapphire starts to absorb to the blue of approximately $300 \mathrm{~nm}$, these windows did not pass 100 percent of our pump beam at 295 $\mathrm{nm}$. This resulted in interfering signals from the creation of $f$-centers in the windows at high pump intensities, thus limiting the size of the signal obtainable in both transient absorption and fluorescence experiments. The last version of the cell constructed utilized fused silica windows $1 / 4$ inch thick (Janos \#A1805-386). This material passes UV light down to $160 \mathrm{~nm}$, allowing higher pump intensities to be used without contributions from excitations in the windows to either transient absorption or fluorescence signals. The compromise 
made in using fused silica is its greater brittleness as compared to the sapphire, and hence a slightly lower maximimum pressure and greater care needed during pressurizing of the cell. 


\section{Chapter 4}

Experimental Data on Relaxation

of Excitons in Condensed Xenon

\subsection{Transient Absorption Data}

1.1 .2 Xenon at $1.8 \mathrm{~g} / \mathrm{ml}(810 \mathrm{psi}$ and $280 \mathrm{R})$ : Transient absorption scans at several wavelengths were taken using the methods described above in the experimental section. The probe wavelengths span most of the visible region as well as the near IR region: $500,550,632,710,740,800,810,830$, and 900 nanometers. Again, each of these wavelengths is the center of a $10 \mathrm{~nm}$ wide portion of the white light continuum. The xenon cell pressure and temperature were fixed at 810 psi and $280 \mathrm{~K}$ for this set of experiments, resulting in a xenon density of $1.8 \mathrm{~g} / \mathrm{ml}$. The effects of changing the density are discussed below. A short 50 ps scan with 0.67 ps steps and a long 4500 ps scan were taken at each wavelength. The short scans are displayed in Figure 11 and Figure 12. The solid lines are fits to the function

$$
\Delta A=C_{1} e^{-\frac{t}{T_{1}}}+C_{2}\left(1-e^{-\frac{t}{T_{2}}}\right)+\text { offset }
$$

The fit parameters are collected in Table II and displayed graphically in Figure 13. Justification of this functional 
Figure 11 and Figure 12: Transient absorption data for xenon at $810 \mathrm{psi}$ and $280 \mathrm{~K}(1.8 \mathrm{~g} / \mathrm{ml})$. Probe wavelengths are shown in each scan. Solid lines are fits to the function $\Delta A=C_{1} e^{-t / \tau 1}+C_{2}\left(1-e^{-t / \tau 2}\right)+$ offset. The function has been convoluted with a Gaussian to represent the laser pulse. The parameters are listed in Table II. 

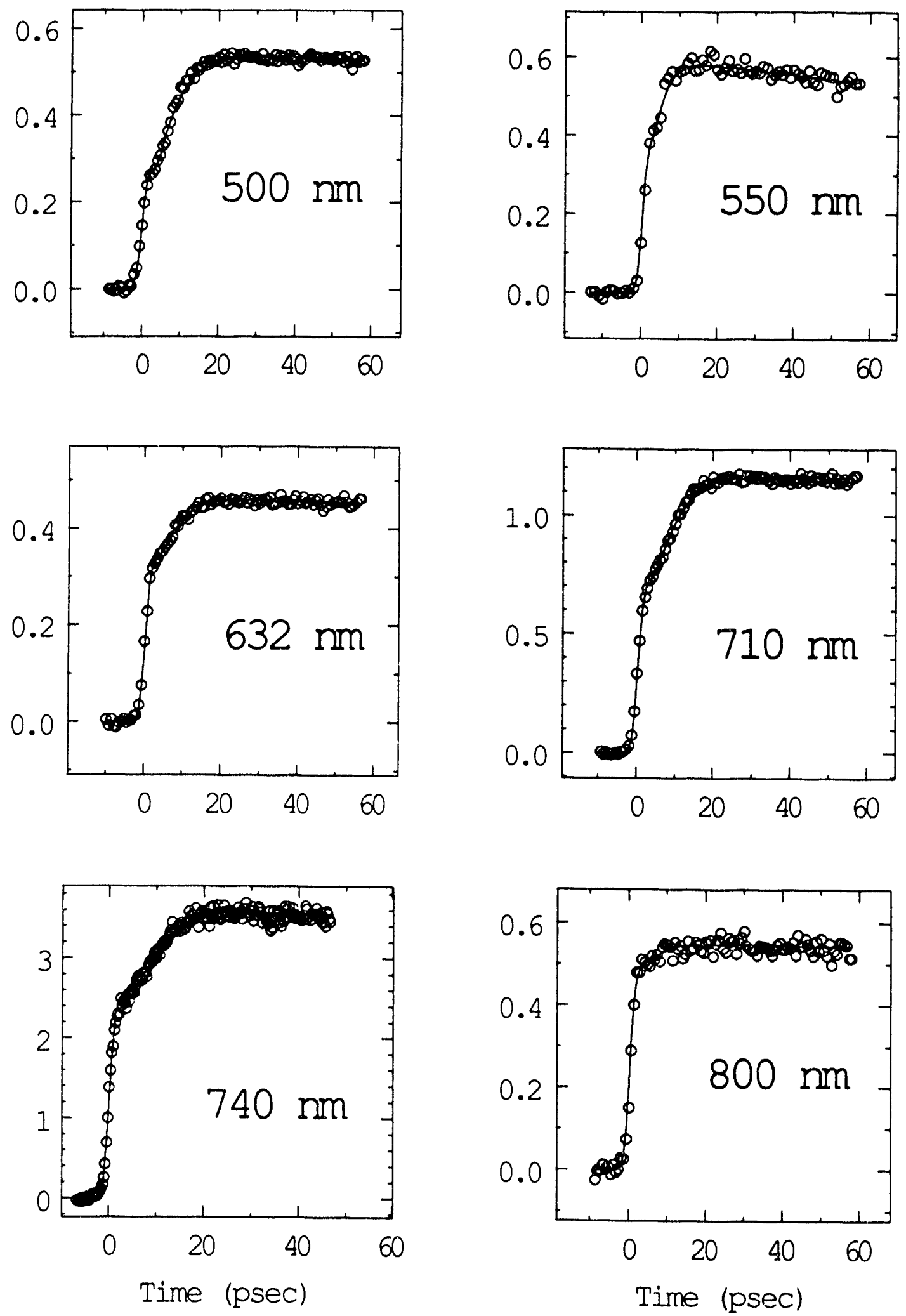

Figure 11: Transient absorption data for $\mathrm{Xe}$ at $1.8 \mathrm{~g} / \mathrm{ml}$ 106 

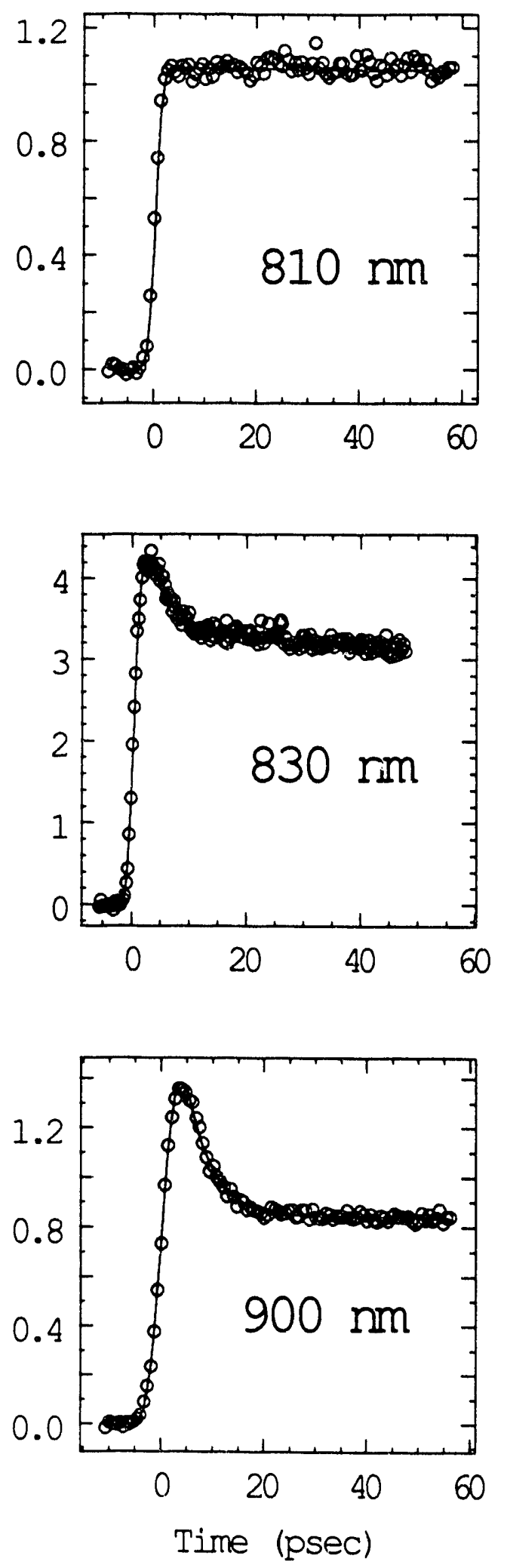

Figure 12: Transient absorption data for Xe at $1.8 \mathrm{~g} / \mathrm{ml}$ 107 
Table II: Fit parameters for exciton dynamics at $1.8 \mathrm{~g} / \mathrm{ml}$.

Fitting parametsrs for wavelength dependence of transient absorption decays for liquid xenon at a density of 1.85 $\mathrm{g} / \mathrm{ml}=8.46 \times 10^{21}$ atoms $/ \mathrm{cm}^{3}$.

fitting function: $\Delta A=c_{1} e^{-t / \tau 1}+c_{2}\left(1-e^{-t / \tau 2}\right)+$ offset

\begin{tabular}{|c|c|c|c|c|}
\hline $\begin{array}{l}\text { probe } \\
\text { wavelength } \\
(\mathrm{nm})\end{array}$ & $\mathrm{Cl} / \mathrm{C2}$ & $\begin{array}{c}\tau_{1} \\
(\mathrm{ps})\end{array}$ & $\begin{array}{c}\tau_{2} \\
\left(\mathrm{ps}^{2}\right)\end{array}$ & $\begin{array}{l}\text { laser } \\
\text { pulse } \\
\text { width } \\
\text { (ps) }\end{array}$ \\
\hline 500 & 0.62 & 0.9 & 6.5 & 1.3 \\
\hline 550 & 0.48 & 2.0 & 5.2 & 1.1 \\
\hline 632 & 0.66 & 2.0 & 4.4 & 1.2 \\
\hline 710 & 0.59 & 2.1 & 6.1 & 1.2 \\
\hline 740 & 0.76 & 2.4 & 5.7 & 1.2 \\
\hline 800 & 0.76 & 2.1 & 2.9 & 1.2 \\
\hline 810 & 1.0 & --- & --- & 1.1 \\
\hline 830 & 0.93 & 3.3 & 2.0 & 1.0 \\
\hline 900 & 2.17 & 4.2 & 3.2 & 1.8 \\
\hline
\end{tabular}

"Data for $810 \mathrm{~nm}$ exhibited a flat response on the timescale of the scan, hence $\tau_{1}=\tau_{2}$.

estimated parameter uncertainties:
$\tau_{1}:$
\pm 1 ps
$\tau_{2}$
\pm 1 ps
laser pulse width: \pm 0.2 ps 
Figure 13: Wavelength trends of the parameters used to fit the $1.8 \mathrm{~g} / \mathrm{ml}$ xenon data. $\tau_{1}$ corresponds to the tunneling time of the free excitons into the trapped state well. $\tau_{2}$ corresponds to the relaxation of the excitons in the trapped state to the fully trapped configuration. The ratio $c_{1} / C_{2}$ is the relative absorption strength of the free excitons to that of the trapped excitons.

$\begin{array}{ll}\text { key: } & \tau_{1} \\ \tau_{2} & \\ c_{1} / C_{2} & x\end{array}$




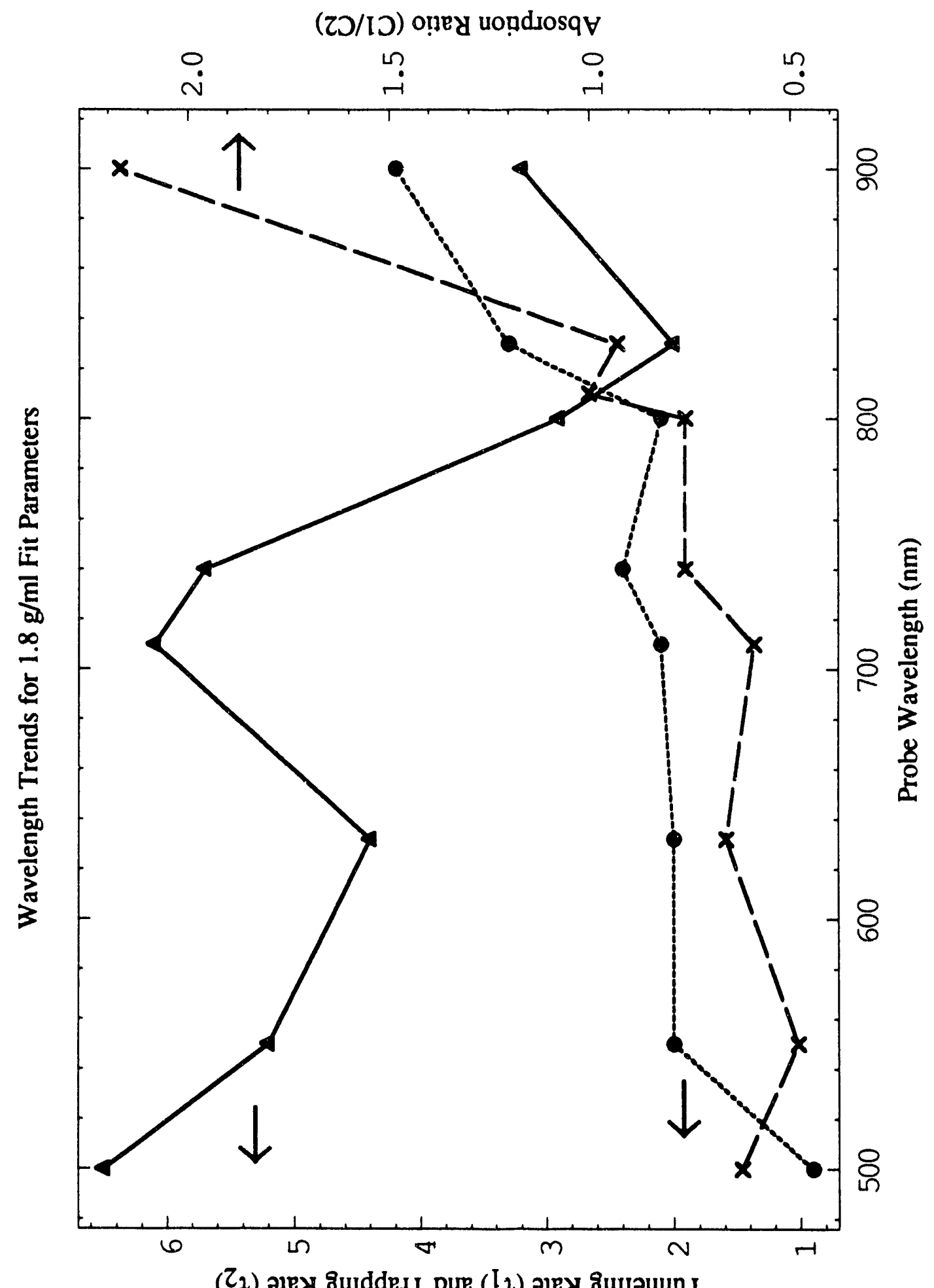

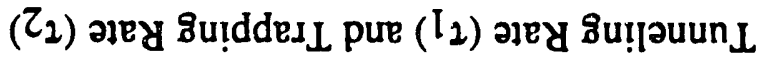

Figure 13: Plot of $1.8 \mathrm{~g} / \mathrm{ml}$ fit parameters vs. wavelength 110 
form is given in the discussion section.

The ratio $c_{1} / c_{2}$ increases monotonically with increasing wavelength. The peak absorption magnitude also increases with an increase in wavelength, but a quantitative measurement of this is not possible as the exact volume of the excited region in the sample is unknown. All of the scans appear to contain three widely differing time scales. The initial rise is pulsewidth limited, indicating a process faster than the resolution of the instrument, which is typically slightly more than 1 ps. The last portion of the rise is due to a slower process of approximately 2 to 7 ps. Depending on the probe wavelength, this manifests itself as either a sharp break in the middle of the rise or as a smooth turnover at the end of the rise. For scans at wavelengths to the red of $810 \mathrm{~nm}$, there is a fast decay of about the same picosecond time scale as the slower component of the rise. All of the scans have a very slowly decaying component with a time constant estimated to be greater than $20 \mathrm{~ns}$. This decay is too long to be resolved under the current experimental setup, and for the purposes of fitting the data, an offset was used to match the long time plateau.

The scan at $810 \mathrm{~nm}$ does not display any of the 2 to $7 \mathrm{ps}$ components, suggesting that $c_{1}=c_{2}$. This is a signature of the possible presence of an isosbestic point in the absorption spectra of the species corresponding to the fast and slow decaying components. As such it is suggestive that only two 
states are involved in the dynamics.

1.1.2 Pressure and Temperature Dependence: Transient absorption data were taken over a modest span of density to sample the liquid, supercritical fluid, and gas phases, within the liquid regime, the pressure could only be varied between about $750 \mathrm{psi}$ and $850 \mathrm{psi}$. Lower pressures resulted in a phase separation into gas and liquid, while higher pressures were not attainable without shattering the cell windows. The wavelengths used in this density dependence study were 500 and 900 nanometers. These data are displayed in Figure 14 and Figure 15 and the fit parameters are listed in Table III. Fits to experimental phase diagram data were used to calculate the densities. ${ }^{87,88}$ The density range spanned in these experiments was 0.11 to $1.85 \mathrm{~g} / \mathrm{ml}\left(5.05 \times 10^{20}\right.$ to $8.46 \times 10^{21}$ atoms $/ \mathrm{cm}^{3}$ ). The data above the critical density for the formation of excitons in liquids, 100 amagats $(2.71 \mathrm{x}$ $10^{21}$ atoms $\left(\mathrm{cm}^{3}\right.$ ), exhibit the same time behavior as in the liquid data in figure Figure 11 and Figure 12. The data for xenon below this density exhibit markedly different behavior, which has been simply fit with an exponential rise and decay to obtain an estimate of the time scales involved.

1.1.3 Pump Frequency Dependence: It was only possible to change the frequency of the UV pump pulse over a small range, limited by the reflectivity bandwidth of the CVI mirrors used 
Figure 14 and Figure 15: Transient absorption data taken for xenon at different densities at $500 \mathrm{~nm}$ and $900 \mathrm{~nm}$. The density of the xenon is indicated adjacent to each scan. Solid lines are fits to the data. The functions used and parameter values are listed in Table III. The functions have been convoluted with a Gaussian to represent the laser pulse. 

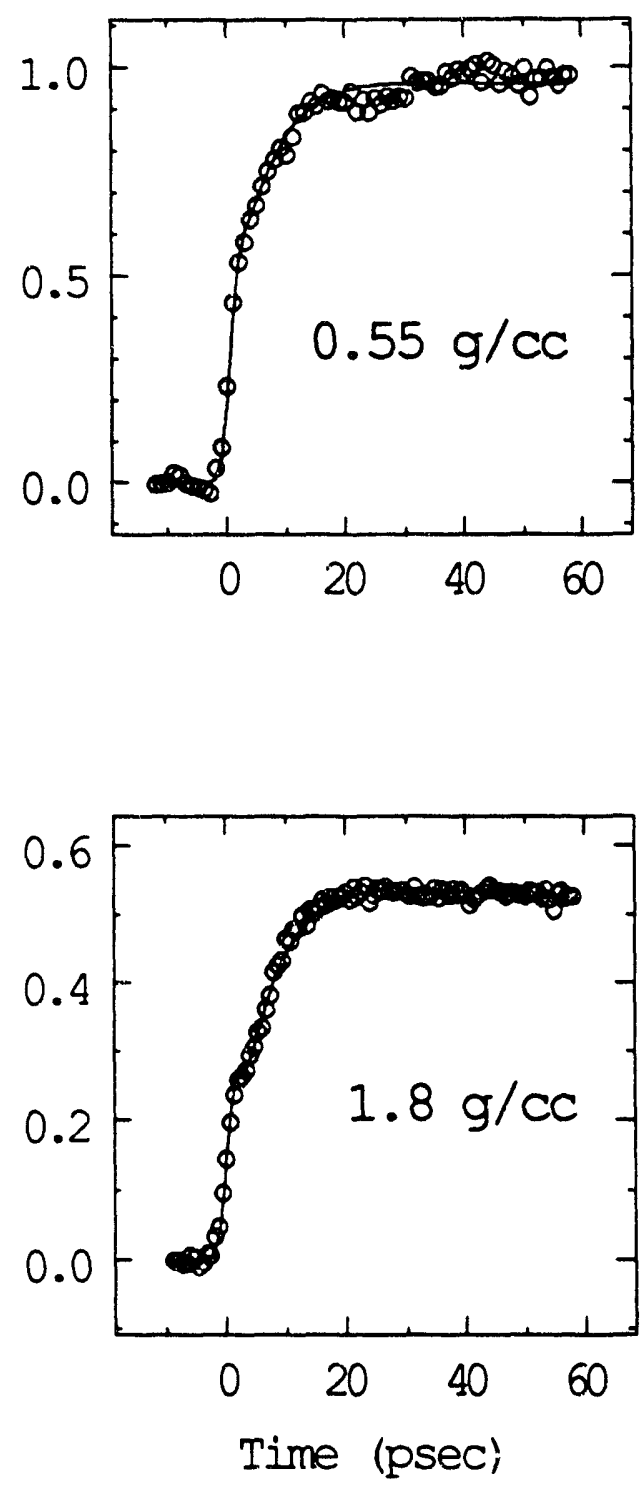

Figure 14: Density dependence of Xe dynamics at $500 \mathrm{~nm}$ 

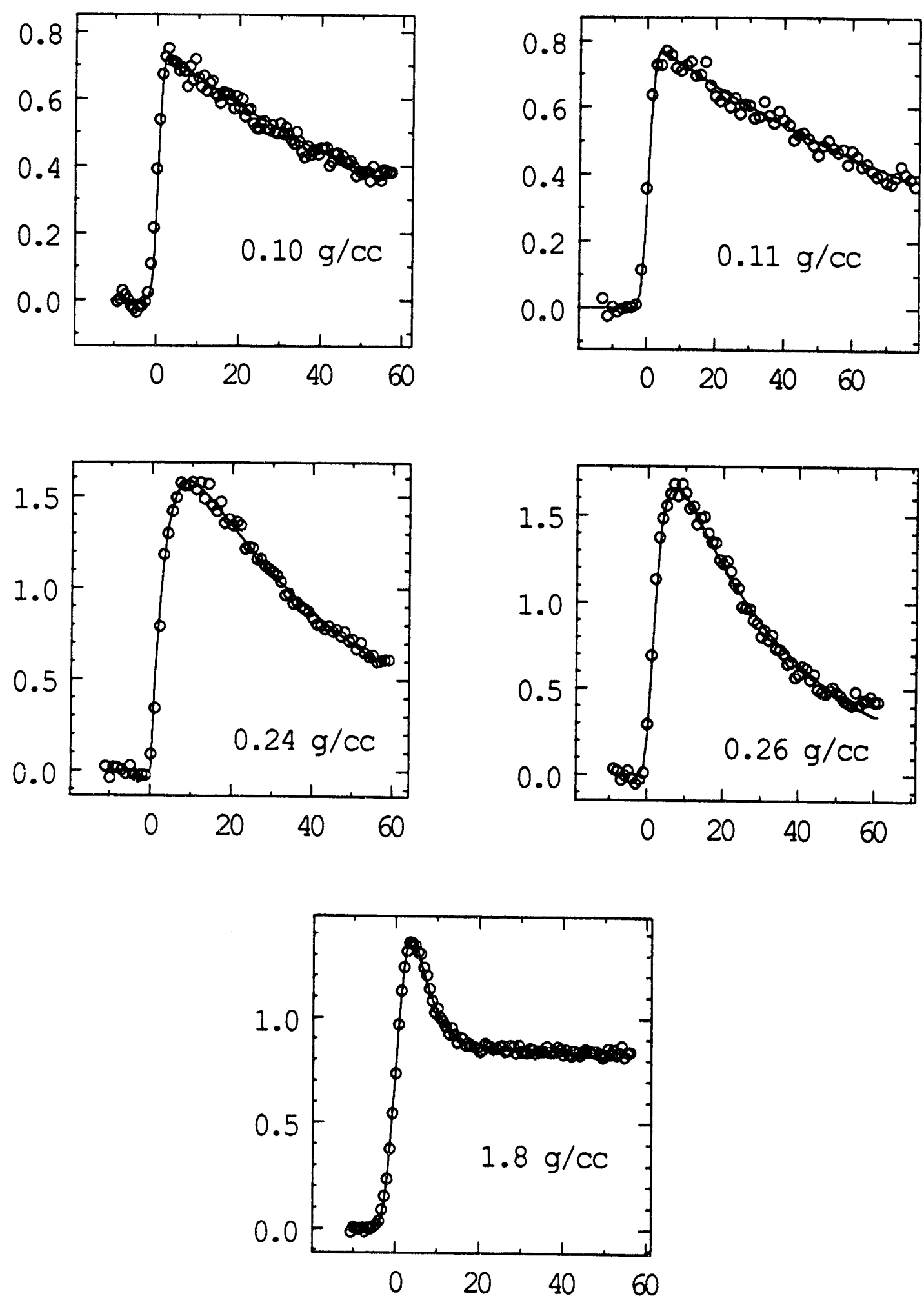

Time (psec)

Figure 15: Density dependence of Xe dynamics at $900 \mathrm{~nm}$ 
Table III: Fit parameters for exciton density dependence

Fits to supercritical fluid and liquid xenon transient absorption data shown in Figure 14 at a probe wavelength of $500 \mathrm{~nm}$ :

fitting function: $\Delta A=c_{1} e^{-t / \tau 1}+c_{2}\left(1-e^{-t / \tau 2}\right)+$ offset

\begin{tabular}{|c|c|c|c|c|c|}
\hline $\begin{array}{l}\text { density } \\
\text { g/ml } \\
\text { atoms } / \mathrm{cm}^{3}\end{array}$ & $\begin{array}{c}\text { temp. } \\
\mathrm{K}\end{array}$ & $\mathrm{c}_{1} / \mathrm{c}_{2}$ & $\begin{array}{c}\tau_{1} \\
(\mathrm{ps})\end{array}$ & $\begin{array}{c}\tau_{2} \\
\text { (ps) }\end{array}$ & $\begin{array}{l}\text { laser } \\
\text { pulse } \\
\text { width } \\
(\mathrm{ps})\end{array}$ \\
\hline $\begin{array}{c}0.55 \\
2.5 \times 10^{21}\end{array}$ & 294 & 0.44 & 2.0 & 5.2 & 1.1 \\
\hline $\begin{array}{c}1.8 \\
8.5 \times 10^{21}\end{array}$ & 280 & 0.62 & 0.9 & 6.5 & 1.3 \\
\hline
\end{tabular}

single exponential fits to gaseous and liquid xenon transient absorption data shown in Figure 15 at a probe wavelength of $900 \mathrm{~nm}$ :

fitting functions: low densities: $\Delta A=C\left(1-e^{-t / \tau 1}\right) e^{-t / 72}$

$$
\begin{aligned}
1.8 \mathrm{~g} / \mathrm{ml}: \Delta A= & C_{1} e^{-t / \tau 1}+C_{2}\left(1-e^{-t / \tau 2}\right) \\
& + \text { offset }
\end{aligned}
$$

\begin{tabular}{|c|c|c|c|c|}
\hline $\begin{array}{l}\text { density } \\
\text { g/ml } \\
\text { atoms } / \mathrm{cm}^{3}\end{array}$ & temp. $\mathrm{K}$ & $\begin{array}{c}T_{1} \\
\text { (ps) }\end{array}$ & $\begin{array}{c}T^{2} \\
\left(p^{2}\right)\end{array}$ & $\begin{array}{l}\text { laser } \\
\text { pulse } \\
\text { width } \\
\text { (ps) }\end{array}$ \\
\hline $4.7 \times 10^{20}$ & 294 & 0 & 75 & 1.1 \\
\hline $5.0^{0.11} \times 10^{20}$ & 280 & 0 & 72 & 1.2 \\
\hline $\begin{array}{c}0.24 \\
1.1 \times 10^{21} \\
\end{array}$ & 294 & 3.4 & 46 & 1.0 \\
\hline $\begin{array}{r}0.24 \\
1.2 \times 10^{21} \\
\end{array}$ & 280 & 2.9 & 31 & 1.1 \\
\hline $8.5^{1.8} \times 10^{21}$ & 280 & 4.2 & 3.2 & 1.8 \\
\hline
\end{tabular}


to turn the UV beam. The data for excitation at $295 \mathrm{~nm}$ and $292 \mathrm{~nm}$ are identical within the errors of the fitting parameters.

4.2 Fluorescence Data: Using the OMA setup described above in the experimental section, fluorescence collection was attempted in the region from 810 to $890 \mathrm{~nm}$. Fluorescence near $830 \mathrm{~nm}$ from a $6 \mathrm{p}$ to $6 \mathrm{~s}$ transition is indicative of the relaxation that follows electron recombination with $\mathrm{Xe}_{2}^{+} .{ }^{89}$ At a xenon density of $1.8 \mathrm{~g} / \mathrm{ml}$ ( $810 \mathrm{psi}$ and $280 \mathrm{~K}$ ) only fluorescence from the sapphire windows of the cell was detected. This appeared structureless and devoid of any of the sharp peaks expected from atomic xenon emission. This would suggest that ionization is not occurring, but as with all negative results, it is not by any means conclusive, and in fact in other experiments in the lab it has been suspected that solvent ionization with the UV beam was a contributing factor in the observed signals. These were not, however, ionizations competing with resonant two photon absorptions as is the case in the xenon experiments. Several improvements could be made to the pressure cell for fluorescence collection as discussed below in the section on future work.

4.3 Discussion of Transient Absorption Data: Two of the UV photons at $295 \mathrm{~nm}$ used as the excitation source are resonant with the $8.42 \mathrm{eV}$ atomic transition from the $5 \mathrm{p}$ to the $6 \mathrm{~s}(3 / 2)$ 
state in the gas phase and the $\Gamma(3 / 2) n=1$ free exciton in the condensed phase (Figure 16). The atomic transition is actually two photon unallowed, and hence the oscillator strength is small, but experiments have shown that the excimers can be excited to the upper region of their wells in this manner. 90,91 In addition, the UV intensities used in these experiments are very high (> $10^{11}$ Watts $/ \mathrm{cm}^{2}$ ), near the breakdown point of the solvent. It is not known how the oscillator strength of this partially allowed process compares with that of the three photon ionization process, but it is likely that the two photon process will dominate. A power dependence study merely revealed that the excitation was in a saturation regime, as the signal was proportional to the excite laser intensity to the 1.5 power. Such behavior in the rare gases has been seen before in multi-photon excite processes. 92 Further experiments will be proposed at the end of this chapter that would resolve this dilemma. For the time being, the possibility of ionization will not be addressed, as it would considerably complicate the fitting process by requiring at least two more parameters. The current data set would not support a unique fit if more parameters were included in the fitting function.

The temperatures of my experiments are much greater than those of previous studies. At $280 \mathrm{~K}$, $\mathrm{kT}$ has a value of 24 mev, very close to the estimated height of the barrier separating the free and trapped exciton wells. As the 
Figure 16: Transitions and processes involved in the transient absorption experiments. Arrows indicate the two UV photons that excite the xenon sample into the free exciton state (or at low densities to the first atomic state (see Figure 1)). This population of free excitons then tunnels into the trapped state well, which is very similar to the outer region of the gas phase excimer $o_{u}$ and $1_{u}$ states. (At low densities the excited xenon atom collides with a ground state partner and forms a highly vibrationally excited excimer.) Transient absorptions of the free state result in transitions to the conduction band, while transient absorptions of the trapped state result in transitions to the other molecular states that correlate to the xenon $6 s$ orbital. These are indicated by the arrows labeled "probe." The tunneling process and the trapping process proceed on a 5 ps time scale as indicated by the labeled horizontal arrows. The lower illustration shows schematically the relative energies involved in the tunneling process. Two populations are represented in the left well by the Gaussian-like traces. The upper one represents a free exciton population at a higher temperature than the bottom one. 
free exciton

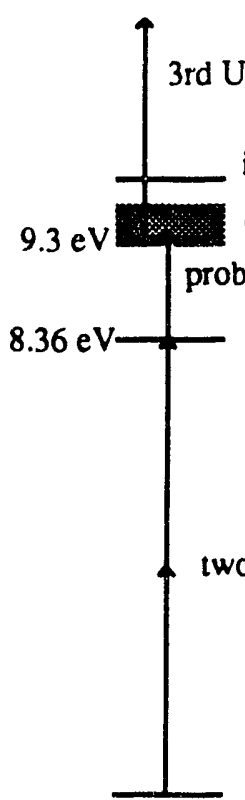

UV photon

ionization potential (solid)

$\Gamma(3 / 2) \quad n=1$ exciton

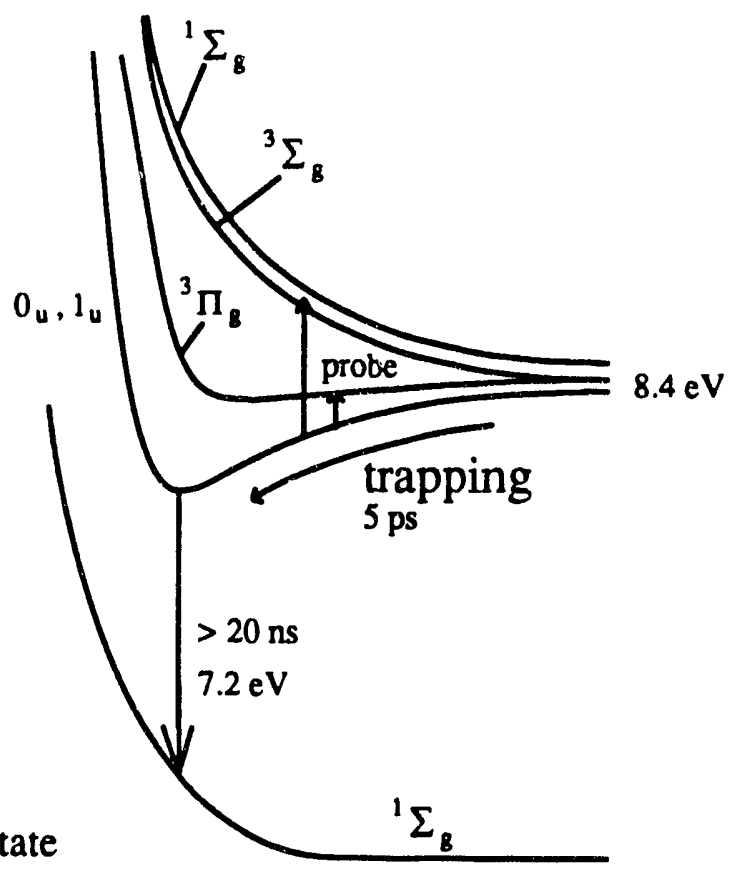

$35 \mathrm{meV}$ barrier in potential of mean force

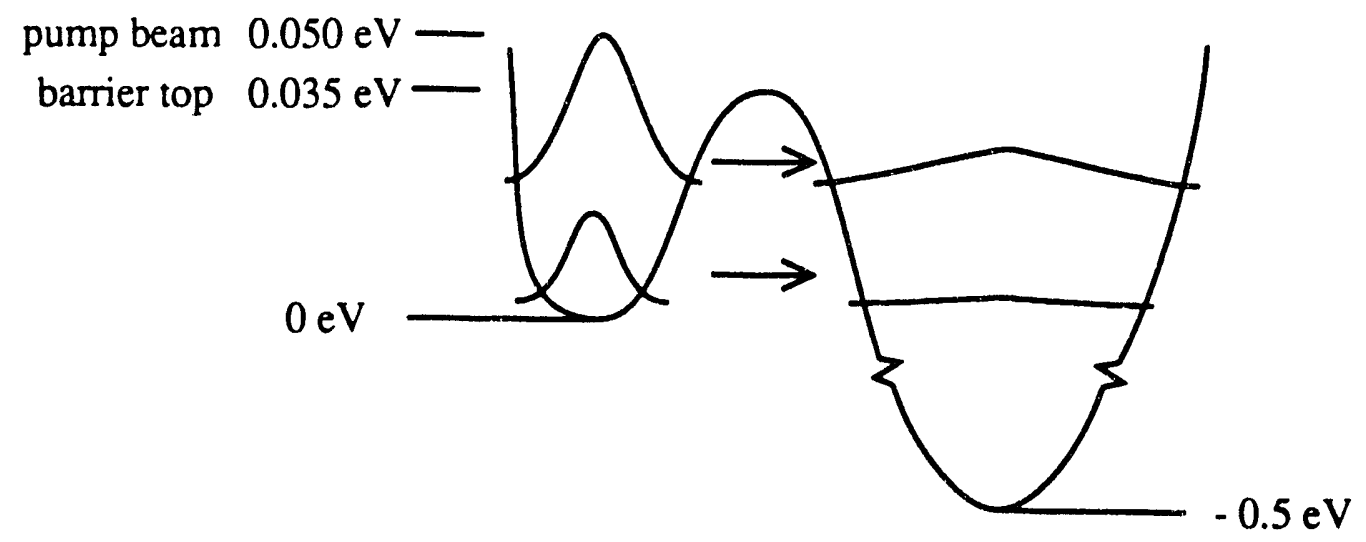

tunneling energetics relative to the bottom of the free state well conduction band

van der Waals ground state

trapped exciton

(excimer)

$\Sigma_{B}$

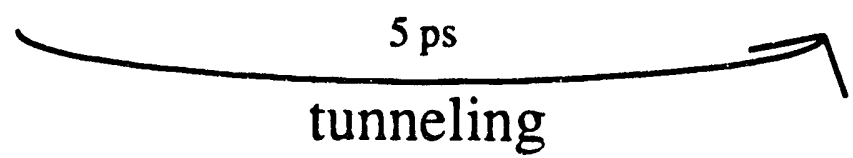

Figure 16: Transient absorption processes and Xe dynamics 
temperature is increased, the free exciton occupies a region of its well closer to the top of the barrier, and so tunneling rates into the trapped exciton well should increase dramatically (shown schematically in the bottom of Figure 16). However, estimates of the thermalization time for the excitons in xenon are only on the 10 to 100 ps time scale; ${ }^{93}$ hence, while this process may be a contributing factor in the picosecond dynamics observed in these experiments, it is not fast enough to fully account for them.

As was mentioned in chapter 2, the barrier between the free and trapped states is altered in the presence of lattice defects in such a way as to decrease tunneling times. The decrease is quite substantial: from 10's of nanoseconds for perfect xenon crystals to just a few picoseconds for solid xenon samples that contain large numbers of vacancy defects. Since the liquid is by nature very disordered, as compared to the solid, the tunneling rates for excitons within it should also be fast. Considering these points, the tunneling in the samples studied here is expected to be very short and on the picosecond time scale.

Martin has estimated that the resonantly enhanced hopping mechanism for trapping should also be fast and predicts a trapping time in liquid xenon at $150 \mathrm{~K}$ of just a few picoseconds. Trapping should be even faster at the higher temperatures of this study.

There are two parameters in equation (23) that are within 
this short picosecond range: $\tau_{1}$ and $\tau_{2}$, the first being a decay time and the second a rise time linked to the long decay. The long decay can be assigned to the population decay of the completely trapped $\Gamma(3 / 2) n=1$ exciton. The preponderance of experimental evidence reviewed above indicates that most of the excited population quickly non-radiatively cascades down to this lowest excited state. The rise time $\tau_{2}$ associated with this state must then be the cooling or vibrational relaxation time of the molecular bond involved in the formation of the $\mathrm{Xe}_{2}$ " trap site as described by Martin. The data exhibit a slight wavelength dependence: in going from $900 \mathrm{~nm}$ to $500 \mathrm{~nm}$ this parameter increases from approximately 2.5 ps to 6 ps. This observed blue shift is a typical signature of vibrational cooling within a molecule, in this case the exciton as it traps into the excimer-like state, and has been seen previously in other molecular systems. ${ }^{94,95}$

The other time parameter, $T_{1}$, is assigned to the time for tunneling to occur between the free exciton and trapped exciton states. The rise of the transient absorption signal from the free state is instantaneous since the excitation is directly to the free exciton state. The decay rate was found to be wavelength dependent with $\tau_{1}$ decreasing with increasing probe energy. It is tempting to assign a cooling phenomenon to this dependence; however, the observed wavelength dependent change in the rate is reversed from the typical trend for a vibrational relaxation process. 
since the energy of the two UV photons used to create the excitons is $50 \mathrm{meV}$ greater than the energy of the free exciton state $(8.41 \mathrm{eV}$ vs. $8.36 \mathrm{eV})$ the population in the free exciton well extends from the bottom of the well to above the barrier. Each segment of this population samples a different point of the barrier as it crosses to the trapped state. The free excitons above the barrier cross directly unhindered into the trapped state well, while those below the top of the barrier tunnel through it at rates dependent on their position within the well: excitons closest to the top of the barrier tunnel at the greatest rate due to the decreased barrier width in this region (again see Figure 16). The transient absorptions from this population spread thoughout the shallow free state well would be able to sample a large portion of the upper energy state, and it is not unreasonable that this would span a wide energy range, as can be seen from the data. Within this context, then, the $r$, values indicate that the free exciton population near the top of the well tunnels quickly through the barrier and absorbs in the green, while the population at the bottom of the well tunnels a bit slower and absorbs in the red. As a final note, the thermalization time for the free exciton is too long to do more than slightly contribute to the tunneling rate. This is also supported by the lack of a change in the dynamics with an increase in the pump energy, which would increase the fraction of the population near or above the top of the barrier that can 
tunnel at the fastest rate or cross directly into the trapped state well.

It is not clear to what state the free population is excited with the probe pulse. If the exciton resides within a small dense cluster within the liquid as suggested by Steinberger et al.,$^{47}$ the probe beam must either cause a transition to a conduction band of this cluster (Figure 16) or ionize the exciton by photoejecting the electron out into the bulk liquid. The band gap in solid xenon corresponding to the $\Gamma(3 / 2) \mathrm{n}=1$ exciton is $9.3 \mathrm{eV}$. If this gap is of similar size in the liquid cluster, then only $0.9 \mathrm{eV}$ of additional energy must be provided by the probe beam over the $8.41 \mathrm{eV}$ already provided by the two UV excitation photons. If the ionization energy of the cluster in the liquid is between the value of $10.7 \mathrm{eV}$ for a cluster of 20 atoms in the gas phase ${ }^{96}$ and the value of 9.33 for the solid, 97 then only approximately $1.5 \mathrm{eV}$ of additional energy is needed for such a transition (note: this is a crude estimate since this is an ionization of the liquid cluster in which the electron goes into the bulk liquid, not the vacuum as is usually the case). The energy required for both the transition to the conduction band and the ionization is equal to or less than the photon energy at all probe wavelengths. In such bound-to-free transitions, the oscillator strength is greatest near threshold and decreases as the energy of the transition increases. Indeed the value of $c_{1}$, which is proportional to 
the oscillator strength of the free excitons, decreases with increasing probe energy.

The trapped exciton population is absorbing to the same states as were discussed in chapter 2 , namely to the ${ }^{3} \Sigma_{0}$ and ${ }^{3} \pi_{0}$ states (Figure 16). Absorptions to a still higher ${ }^{3} \Sigma_{g}$ state corresponding to the core hole transition lie to higher energies than were probed in these experiments. The general trends of $c_{1}$ and $c_{2}$, the relative absorption strengths of the free and trapped exciton states, agree with the literature. The absorption spectrum of a trapped exciton in xenon peaks in the red, and it must be that the free exciton absorption band peaks even further to the red. The absorption spectra of the free and trapped excitons cross near $810 \mathrm{~nm}$, leading to a time independent transient absorption signal on the 10's of ps time scale. This is known as an isosbestic point, a signature in the spectra of a coupled two species system. The free exciton is decaying into the trapped exciton, but since they have the same absorption cross section at 810 , the total absorption does not change in time.

The density dependence study reveals two very different behaviors above and below the density threshold for the appearance of the $n=1$ exciton. As measured by steinberger et al. this value is approximately 100 amagats, or $2.7 \times 10^{19}$ atoms $/ \mathrm{cm}^{3}$. Below this density the xenon merely forms excimers as it does in the gas phase, while above this density the free excitons are excited within the small high density clusters 
that exist momentarily in the fluctuating liquid. These then relax into the trapped exciton state via the mechanism described by Martin (Figure 17). The data strongly suggest that this partitioning does in fact occur. In the low density region, the relaxation time scales with density, as might be expected from a relaxation process where single collisions with ground state partners would dominate the relaxation of the excimer. In the high density region a doubling of the density does not produce any change within the noise of the fit parameters. It must be the case in xenon that a cluster dense and ordered enough to support an exciton relaxes with Martin's hopping mechanism already operating near its maximimum rate, and so relaxation is largely unaffected by changes in the bulk density. Indeed, Martin calculates rates for all of the rare gases over the temperature range of $20 \mathrm{~K}$ to $150 \mathrm{~K}$, and they all exhibit relaxation rates such that trapping is completed within 1-5 ps.

As one final note, although the fitting parameters for the transient absorption decays did not change with an increase in the xenon density once the system was above the critical density threshold for exciton formation, a spectral shift of the exciton bands is expected to accompany such an increase, as was explained in chapter 2. Future work will attempt to cover a broader density range and verify this.

In summary, this is the first study of exciton behavior in xenon on the picosecond time scale and at room temperature 
Figure 17: Relaxation mechanisms of excimers in the gas phase and low density fluids and of excitons in the high density fluid trapped state well. The relaxation of an excimer via single collisions with ground state atoms is illustrated in the top half of the figure. The resonant energy transfer hopping mechanism proposed by Martin is illustrated in the bottom half. The excimer is the darkely shaded pair of overlapping circles, with the degree of relaxation indicated by the amount of overlap. Ground state atoms are the medium shaded circles. The light circles with the arrows inside them represent an atom about to collide with the excimer in the liquid. During the collision this colliding atom displaces one of the atoms in the excimer. The displaced atom is shown as a light circle with no arrow. The propagation or hopping that results is indicated by the migration of the excimer across a small sample of liquid in the four frames in the bottom half of the figure. 

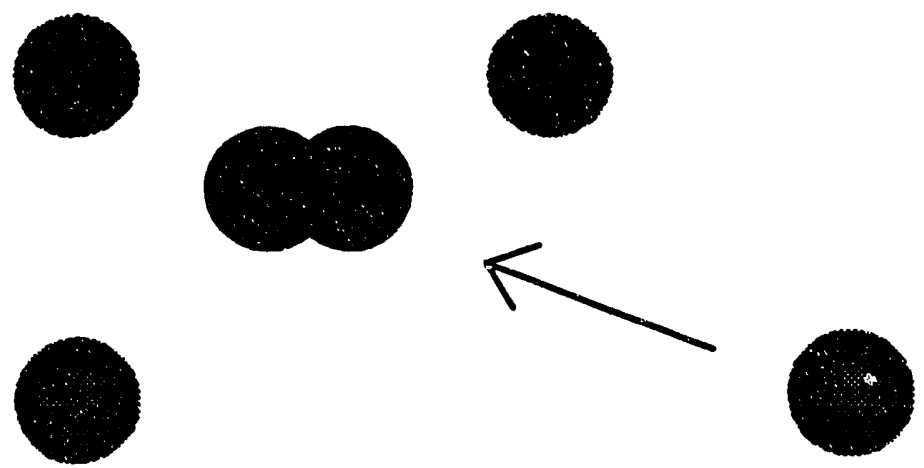

relaxation by single collisions in the gas and low density fluid phases

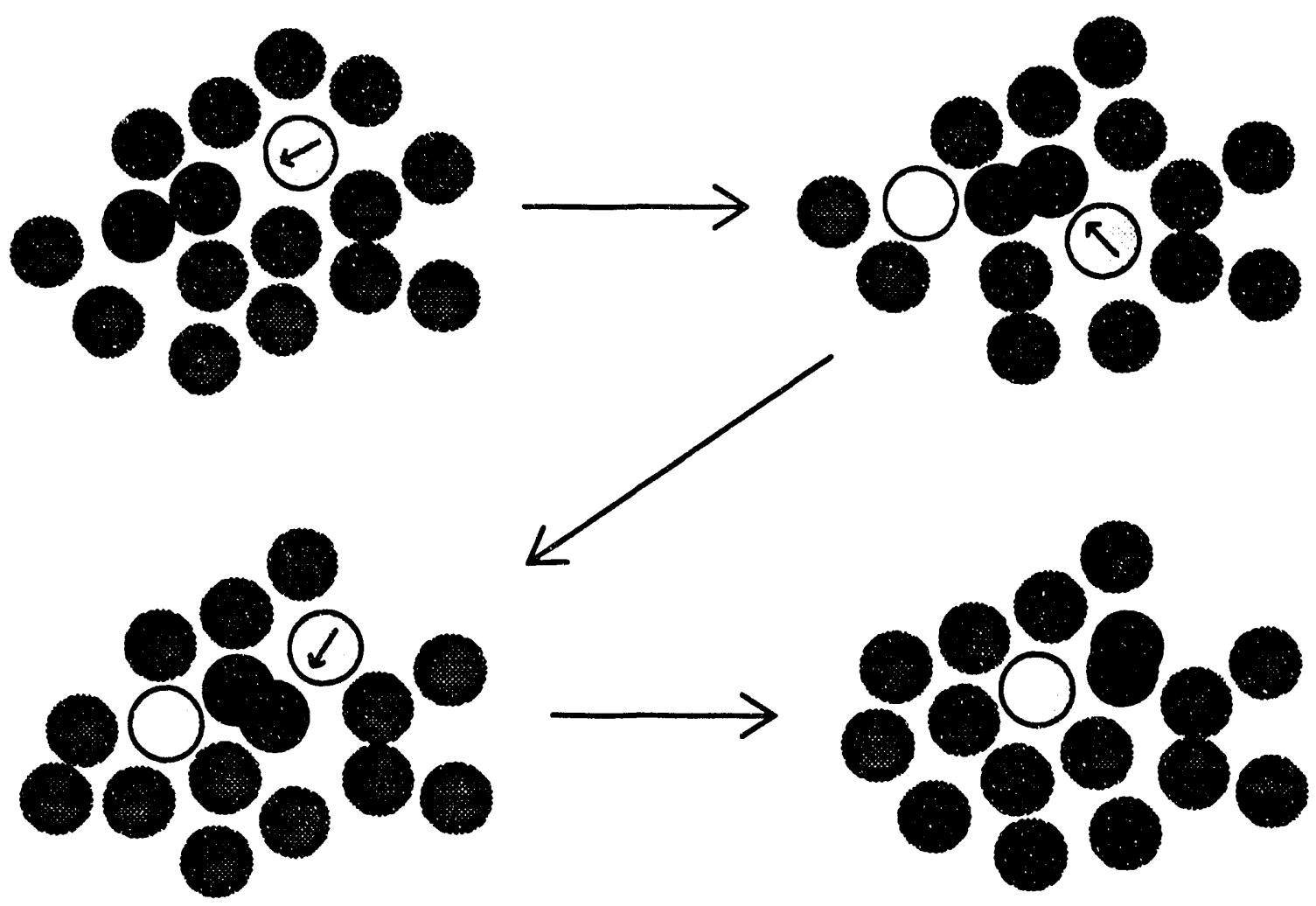

Trapping of exciton into excimeric state via

Martin's hopping mechanism in high density fluids

Figure 17: Mechanism of exciton trapping proposed by Martin 
under high pressure conditions. Both trapping and tunneling of the $\Gamma(3 / 2) n=1$ exciton have been directly observed under these conditions for the first time. As expected from experiments on crystalline xenon, both of these rates were much faster in the liquid than in the solid. In addition the tunneling and trapping were both found to occur on approximately a 5 ps time scale in agreement with the theory for tunneling by Kmiecik and schreiber and with the theory of trapping by Martin. The absorption spectrum of the exciton after tunneling shifts to the blue as trapping occurs, indicating that vibrational cooling of the molecule-like trapped state is part of the relaxation process. Evidence of a density threshold for the formation of the excitons in xenon is evident in the time scales of the relaxation, and indeed the two regimes above and below this threshold density exhibit very different dynamical behavior. Below the critical density the relaxation rate scales with density as is expected for the cooling of a molecule that is driven by single collisions with the bulk solvent, and above the critical density the relaxation rate appears to be independent of density, indicating that the resonant energy transfer mechanism proposed by Martin is working near maximum efficiency in this density range, and is thus influence very little by density changes.

1.4 Suggestions For Future Work: Needless to say, there are many unanswered questions remaining after this work and more 
experiments to do to answer them. Repeating the transient absorption experiments with an electric field across the sample as in Kubota et al.'s experiments would answer several questions. First, the collection of current in and of itself in the presence of the excitation beam would indicate that three photons are being absorbed and that some of the transient signal is due to the recombination processes of the resulting ions and electrons. The xenon ion behaves in much the same way as the excited atom: it also forms excimers bound by roughly the same energy as the neutral excimers, and it has nearly the same absorption spectrum as we].1. Second, the recombination of the electrons and ions could be observed by monitoring the current since the probe beam could be used to re-ionize the atoms, resulting in an measurable increase in current. Both the lifetime and energy relaxation as a function of time of the trapping excitons could be measured in this type of two photon photoemission experiment. These types of experiments are quite difficult as the transient currents are strongly affected by contaminants, stray capacitances in the cell, and space charge effects.

It would also be invaluable to obtain time resolved luminescence data. Since the luminescence energy is very indicative of the identity of the species emitting, a decay with the same picosecond rates as those seen in the transient absorption experiments would make the assignments suggested for that data much more certain. The free exciton should emit 
near $8.4 \mathrm{eV}$, while the trapped exciton should emit near 7.2 ev. Unfortunately the cell constraints needed to contain the high pressure make collection of luminescence rather difficult. The bore of the cell is small and long, necessitating fluorescence collection along the same axis as the laser pump beam. As such the geometry is far from ideal and could be improved by the addition of a window at 90 degrees to the present two. This, however, complicates the cooling jacket construction. The small bore means there is a small collection angle--less than 0.05 steradians. This could be increased somewhat by using a larger and thicker window. An additional problem is the fact that the source of the fluorescence photons is not a point, but rather the entire excitation profile in the xenon. Since the UV beam is along the collection axis, the image created by the fluorescence is $1 \mathrm{~cm}$ deep, the width of the cell. This means that a single focal length lens will not be able to collimate all of the fluorescence. Again, collection from the side in conjunction with use of a cylindrical collection lens would alleviate this problem. Also both sapphire and quartz windows begin to absorb well below the energy of the luminescence. Lif windows would not sustain the high pressure gradient. The inner surface of the windows would have to be covered with some type of scintillator to convert the VUV luminescence into visible photons that could then escape the cell. This would prevent picosecond resolution, however. 
Emission collected at the other end of the spectrum near $828 \mathrm{~nm}$ would be helpful in determining the path of relaxation of the hot dimer produced in electron recombination. ${ }^{89}$ The emission at $828 \mathrm{~nm}$ occurs from a $6 \mathrm{p}$ state to a $6 \mathrm{~s}$ state shortly after the dimer curve crosses onto a dissociative molecular state following the recombination. It may very well be that at the high densities of these experiments the dissociation would be arrested before completion by $a$ quenching collision with the surrounding atoms. This would result in a curve crossing onto the bound state that lies just below the dissociative state in energy at large internuclear separations. In this case even though ionization is occurring, the 828 emission would not be seen. In fact the Iuminescence seen by Kubota is much less than that expected from gas phase results. ${ }^{98}$ Future experiments should be done to vary the density over a greater range, and certainly up into the solid region of the phase diagram. For this a much more robust cell will be need to be designed utilizing Nova Swiss and High Pressure Inc. parts that have been thoroughly cleaned to remove all oil. 


\section{Appendix 1}

\section{A Note on the effects of Impurities in the Xenon}

The xenon used in these experiments was grade 4.5 (99.995\% Xe) obtained from Airco and used without further purification. The dominant impurity is krypton, present at approximately 25 ppm. The mixed excimers $\mathrm{Xe}-\mathrm{Kr}$ and $\mathrm{Xe}-\mathrm{Kr}{ }^{*}$ exist, ${ }^{20}$ but at this level one does not expect them to dominate the spectrum or influence the decays observed. Also, as the excited states of $\mathrm{Kr}$ are higher in energy than those of Xe, they should not act as low energy traps for the Xe excitons. The other impurities in the Xe were $\mathrm{N}_{2}$ at $5 \mathrm{ppm}$ and $\mathrm{CO}_{2}$, hydrocarbons, and water all at $1 \mathrm{ppm}$ or less. It has been suggested that some of the bands seen in luminescence spectra in the region of 4-6 eV are due to formation of $x e 0,99$ but again not at the levels of $O_{2}$ in these experiments.

What has been noticed aftex filling the cell with xenon and cycling the xenon in and out of the cell several times is an accumulation of oils on the cell windows. This was identified as diffusion pump oil in a mass spectra analysis. The presence of this oil affected only the long time components in the decays at all wavelengths. The oil must act either as a trap of the $\Gamma(3 / 2)$ exciton or it itself has an excited state decay which absorbs in the visible. The result was a reduction in the long time decay constant from a value 
of greater than $20 \mathrm{~ns}$ to in some cases as little as $3 \mathrm{~ns}$. As with most impurities, the amount of oil in the cell was not controllable, and so this change in the long decay occurred each time the cell was emptied and refilled with no reproducability. There was, however, a trend in the lifetimes of the long decay for the first dozen or so fillings of the cell as shown in Figure 18. Between fillings the xenon tank on the cell was only warmed enough to produce adequate pressure to fill the cell. With each trapping and filling cycle the xenon entering the optical cell became more and more pure as the oils trapped out on the solid core of xenon left behind in the bottle. This manifested itself in dramatic increase in the decay constant, with the value approaching the literature value of 32 nanoseconds as quoted above. Eventually, however, the cell was filled enough times from a warm tank (as is the case for the first filling of each day), and the oil accumulated at a high enough level in the cell so that trapping and filling did not remove it again. At this point the cell had to be dissassembled and cleaned. 
Figure 18: Effect of diffusion pump oil impurities on the long time decay constant. Between each scan the cell was emptied and refilled with xenon. No other changes occurred. 

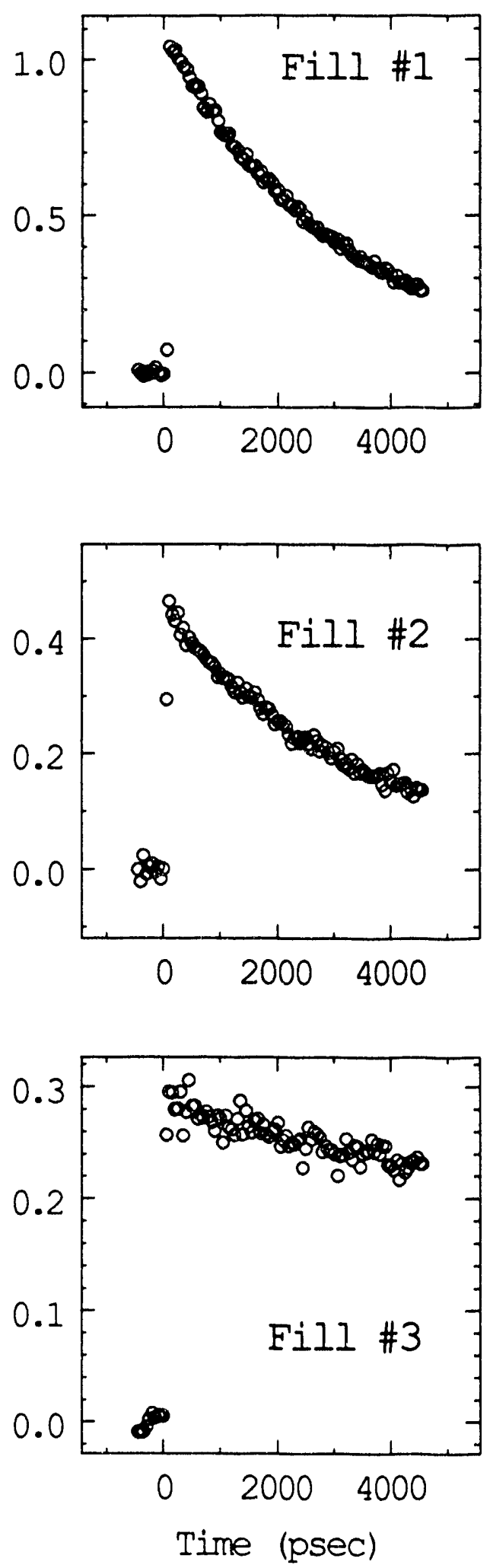

Figure 18: Effect of oil impurities on long decay 136 


\section{Appendix 2}

\section{Data collection subroutines (c programming language)}

\section{Bubroutine adc.c:}

/*subroutine adc.c

This subroutine reads the $A / D$ converter used with the $10 \mathrm{~Hz}$ amplified picosecond laser system. and passes the data acquired there to the arrays in "Nice", the data collection routine written by Dan Russell. ${ }^{100}$ As such it replaces the assembler code in "Nice," contained in the subroutine "dani," which was designed for data acquisition on the cpm laser system in the group. The sequence of operations is the following. First the function readadc looks for a Look-At-Me (IAM) from the $A / D$, signifying that it has a piece of data. Then the data is read off the 0,1 , and 2 channels of the $A / D$ into the array data[k], and the A/D is cleared. The background levels, or pedestals, are subtracted from the data, and the results are checked to see if they lie between the lower and upper bounds. If they are in bounds, the elements of data[k] are passed to corresponding elements of dtemp[k]. This process is continued until the $A / D$ has been read "shots_step" times, where shots step is equal to the number of shots per stage step. A ratio (norm) of the first $A / D$ channel datum to the zeroth $A / D$ channel datum (signal/reference) is computed and passed to dtemp[3]. Control is then passed back to the main program along with the contents of dtemp[k]. The main program then stores the data, moves the stage, and calls this subroutine again. The reader is referred to pp. 5-3 to 5-6 in the dsp technical reference manual for the 6001 CAMAC crate controller for more information on the CAMAC commands and examples of their use.*/

\#include "dan.h"

void readadc (double dtemp[], int *e) (

/* shots_step is the number of laser shots acquired per stage step. Low and high are the lower and upper bounds respectively. For the linear region of the $A / D$, these have been experimentally found to be 350 and 900 by Keenan Brown. Adchan [3] is an array that labels the $A / D$ channels. AD is the crate position of the $A / D$, set in the routine kmain to be 10 . Pedestal[3] are the pedestals set in the setup file.*/

extern long int shots_step;

extern int low, high, adchan[3];

extern int $A D$, pedestal [3];

$I^{*} \quad i, j$, and $k$ are respectively counters for shots taken at 
each stage step, a dummy counter set to specify how long to look for data, and the number of $A / D$ channels read. $L$ is the return of CAMAC command CAML(L) telling the program the highest priority pending IAM. LAM is value of the crate slot of the device with a LAM signal, in this case it will be 10. fun is the function set for the CAMI and CAMO commands. Q and $X$ are status parameters indicating success or failure of a CAMAC command. numadchan is the number of $A / D$ channels to be read; it can be 1,2 , or 3 and is set to 3 . */

int $i, j, k, L, L A M$, fun, $Q, x$, numadchan;

long int data[3];

int out;

/* norm is the ratio between the signal and reference channels, here set to $A / D$ channels 1 and 0 . For absorption experiments it will be the transmittance. */

double norm;

$i=j=k=L=L A M=$ fun $=Q=X=0$;

/* *e is a pointer to an error code specified in "New."

*e=0;

$\operatorname{data}[0]=\operatorname{data}[1]=\operatorname{data}[2]=0$;

norm $=0$;

$\operatorname{dtemp}[0]=\operatorname{dtemp}[1]=\operatorname{dtemp}[2]=\operatorname{dtemp}[3]=0$;

numadchan $=3$;

/* Loop for taking shots_step number of data points. */

$$
\text { for }(i=0 ; i<\text { shots_step; } i++)(
$$

$/$ * out is a flag for a shot out of bounds. */

$$
\text { out }=0 \text {; }
$$

/* dummy loop limiting the amount of time that the program will look for a LAM. If no LAM is found after 10000 iterations an error message to that effect is displayed. */

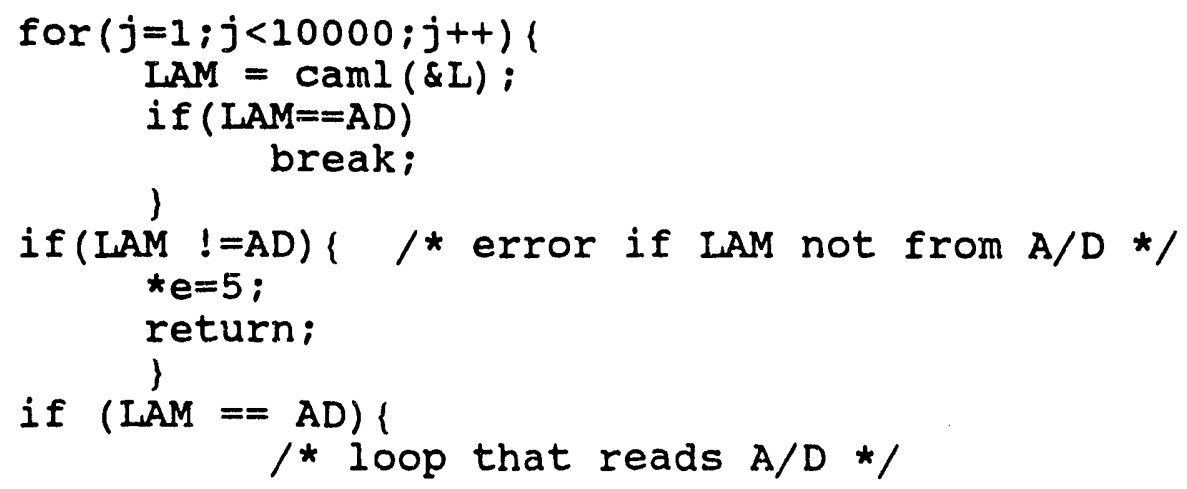




$$
\begin{gathered}
\text { for }(k=0 ; k<\text { numadchan; } k++)( \\
\text { fun }=0 ;
\end{gathered}
$$

/* The CAMI command reads data from the $A / D$ in slot $A D$. fun $=0$ specifies read. adchan [k] is the $A / D$ channel read. The datum read is returned in data[k]. Q and $x$ must both equal 1 for successful read. $\quad$ /

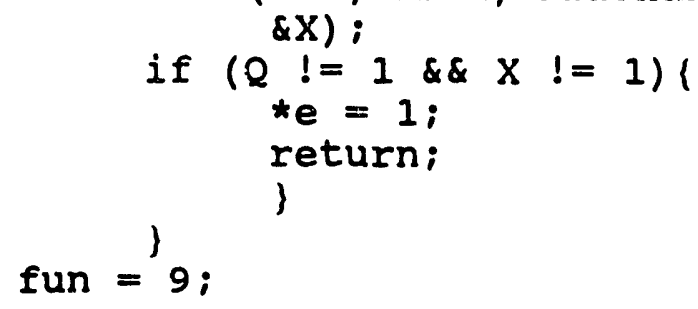

/* The CAMO command writes to the $A / D$. fun $=9$ specifies clearing the $A / D$. The clear is started at adchan[0] and clears all channels sequentially. 0 holds place for no returned data. */

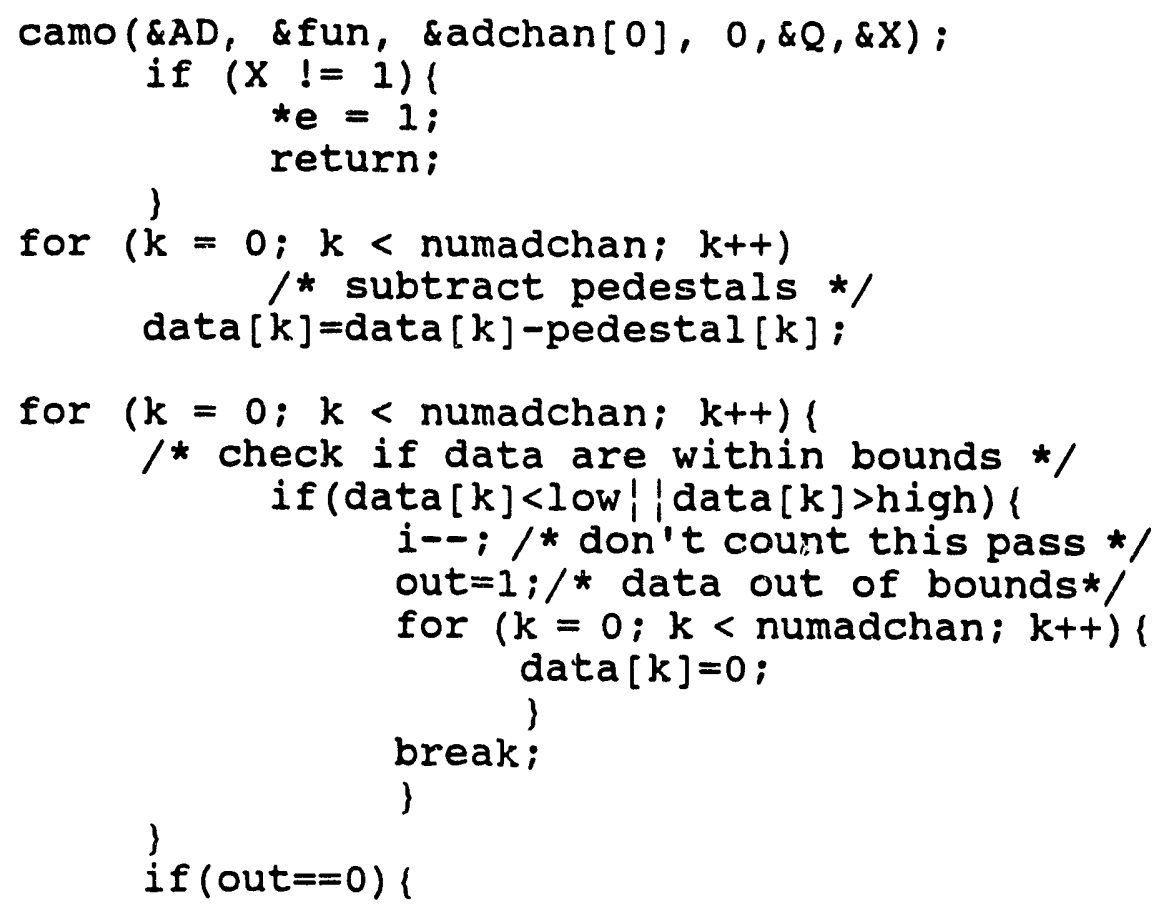

/* add data to other shots taken at this stage position and if data taken for channels 0 and 1 calculate norm and add this to dtemp[3]. */

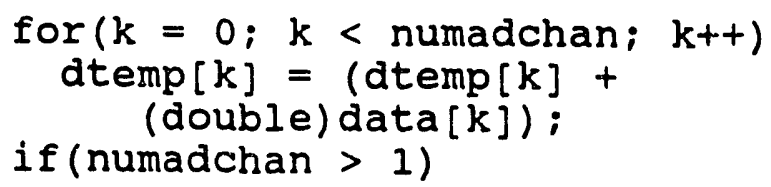




$$
\begin{gathered}
\text { dtemp }[3]= \\
\text { ((double)data }[1] /\left(\begin{array}{c}
\text { double) data }[0]) \\
\text { +dtemp }[3] ;
\end{array}\right. \\
1 \\
/ * \text { end of } i \text { loop taking shots_step data shots } * /
\end{gathered}
$$

1 * divide out number of shots taken so scans can be added together later. */

$$
\begin{aligned}
& \text { for }(k=0 ; k<\text { numadchan; } k++) \text { dtemp }[k]=\operatorname{dtemp}[k] /(\text { double) shots_step; } \\
&\text { if (numadchan }>1) \\
& \text { dtemp }[3]=\operatorname{dtemp}[3] /(\text { double) shots_step; }
\end{aligned}
$$

return;

\}

$$
\text { /* passes control back to main program */ }
$$




\section{subroutine adcshut.c:}

/* subroutine adcshut.c

This subroutine works the same way as the previous one, adc.c, with the provision of operation with a shutter in the excite beam. Such an option is useful to eliminate dc absorption signals caused by photoproduct or "crud" on the windows or in solution of the sample cell due to the photodegradation of the sample by the excite beam. Subtraction of data taken with the excite blocked from data taken with the excite unblocked results in the desired removal of the dc ground state absorption signal from the photoproduct (unless of course the photoproduct has a transient absorption at the probe wavelength as well).

In this routine, data[k] used in adc.c is replaced with rawopen[], rawclosed[], dataopen[], and dataclosed[]. Subtraction of the pedestals for each channel of data for the corresponding elements of rawopen[] gives dataopen[]; similarly for rawclosed[] and dataclosed[]. The contents of dataclosed[] are subtracted from dataopen[] and put into dtemp[] for return to the main program. The number of shots taken per stage step is specified again by shot_step, and the number of shots taken with the shutter open, which equals the number taken with the shutter closed, is given by the variable shuttershots. See the comments in adc.c for further information, especially regarding the CAMAC commands. */

\section{\#include "dan.h" \\ \#include <bios.h>}

extern long int shots_step;

extern int $A D$, pedestāl[3], adchan[3];

extern int low, high:

extern int shuttershots:

void readadc_shutter (double dtemp [], int *e)

$$
\text { 1 }
$$

int $i, j, k, L, L A M$, fun, $Q, x, n$, rawopen[3] ;

int rawclosed [3];

int numadchan:

double dataopen [4], dataclosed [4];

double norm, etmp;

int out;

$i=j=k=I=n=$ fun $=Q=X=0$;

rawopen $[0]=\operatorname{rawopen}[1]=$ rawopen $[2]=0$;

rawclosed $[0]=$ rawclosed $[1]=$ rawclosed $[2]=0$;

dataopen $[0]=\operatorname{dataopen}[1]=$ dataopen $[2]=0$;

dataclosed $[0]=$ dataclosed $[1]=$ dataclosed $[2]=0$; 


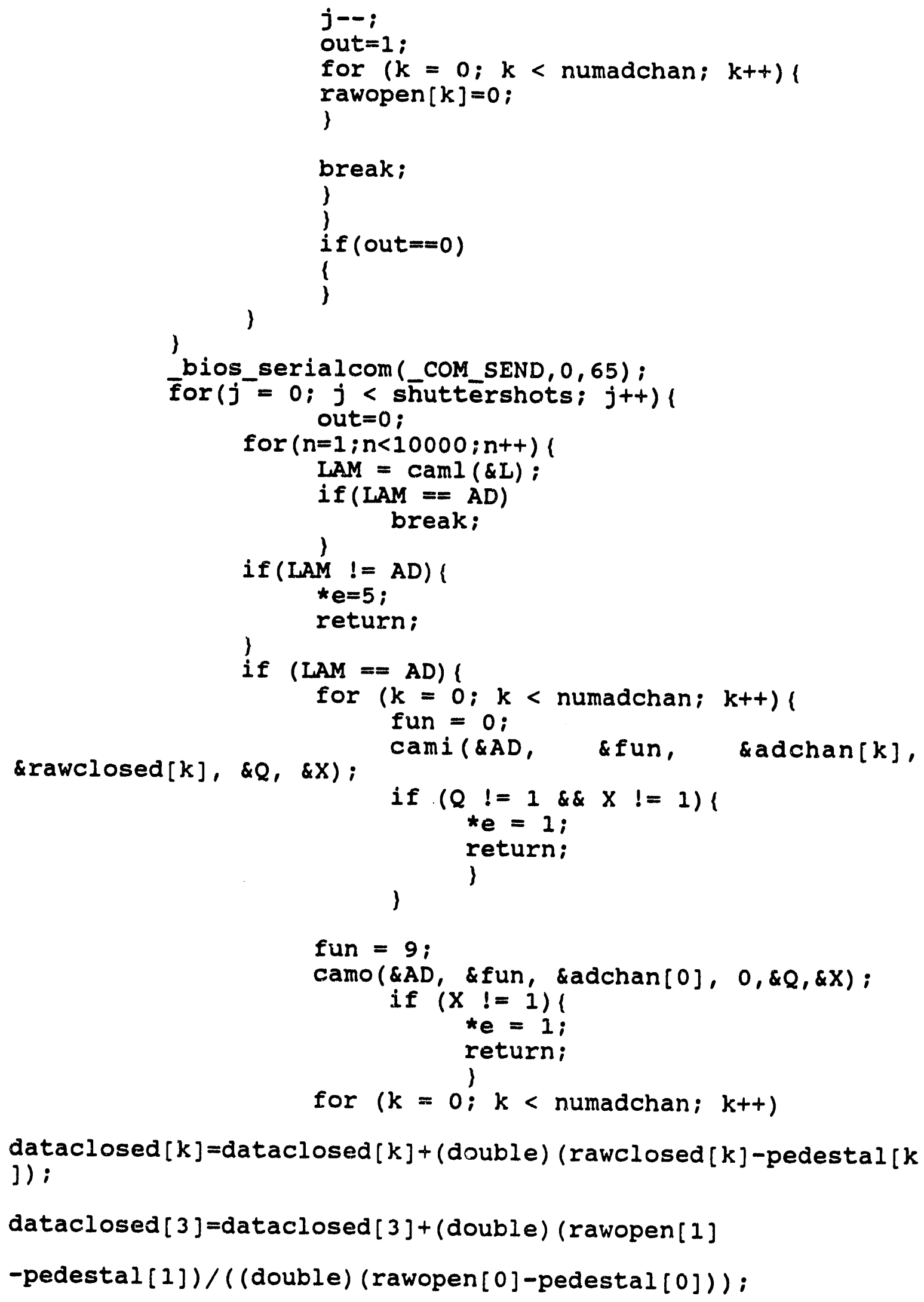




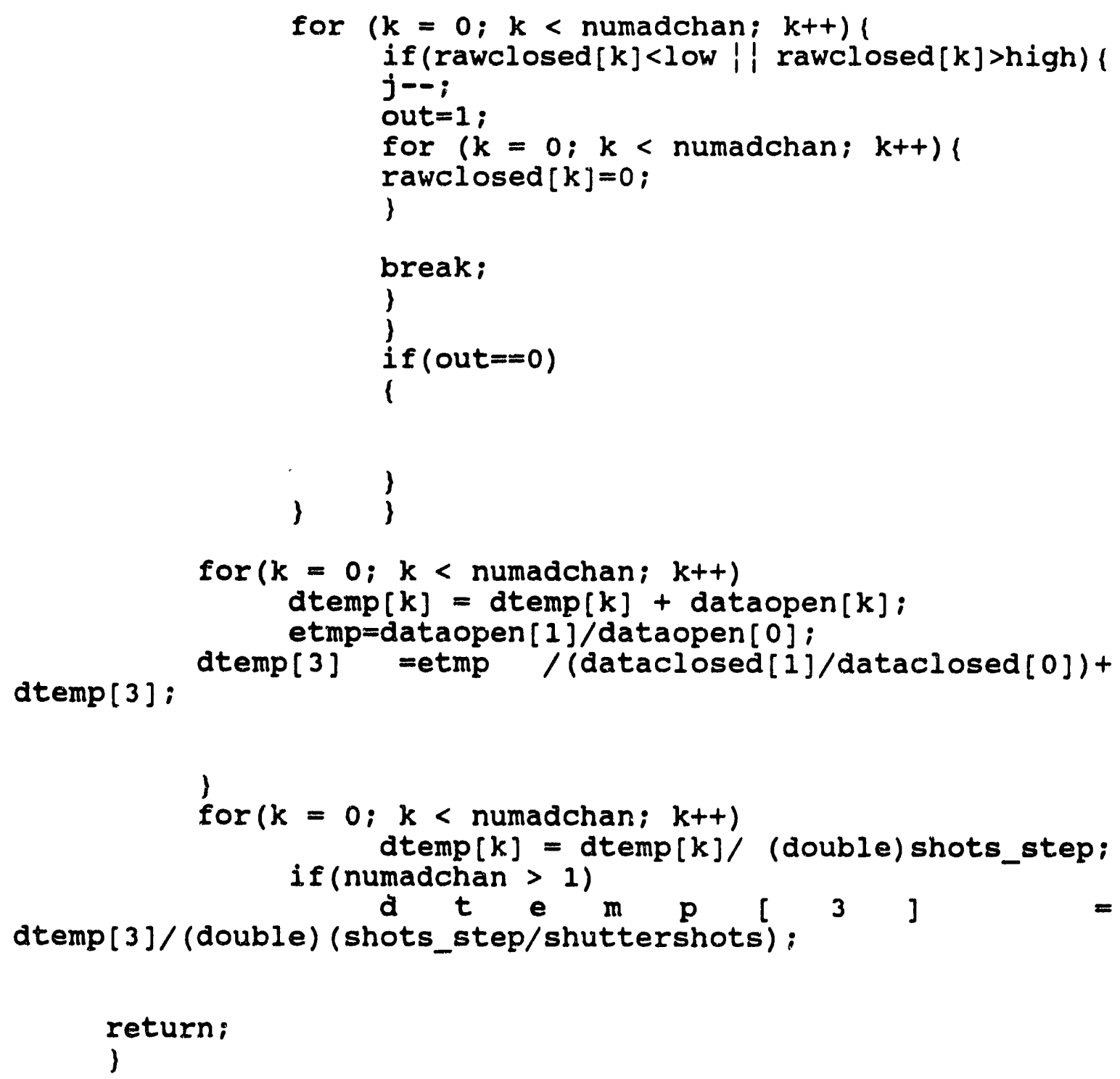




\section{Bubroutine eric.c:}

/* subroutine eric.c

This subroutine calculates the standard deviation for the three different pieces of data, channel 0 , channel 1 , and norm, which is the ratio of channel 1 to channel 0 . When the run norm option is selected from the operating menu, this function is called. Normtemp[0] contains the sum of channel 0 , and normtemp[0+3] contains the sum of the squares of channel 1. normtemp[1] contains the sum of channel 1 and normtemp[2] contains the sum of norm. See comments in adc.c for information on the CAMAC commands. */

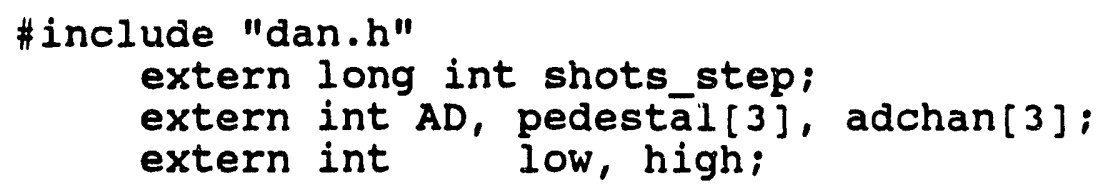

neric(double *normtemp, int $\star e)$ (

int $i, j, k, I, L A M$, fun, $Q, x$, numadchan;

long int data[3];

int out;

double norm, etmp;

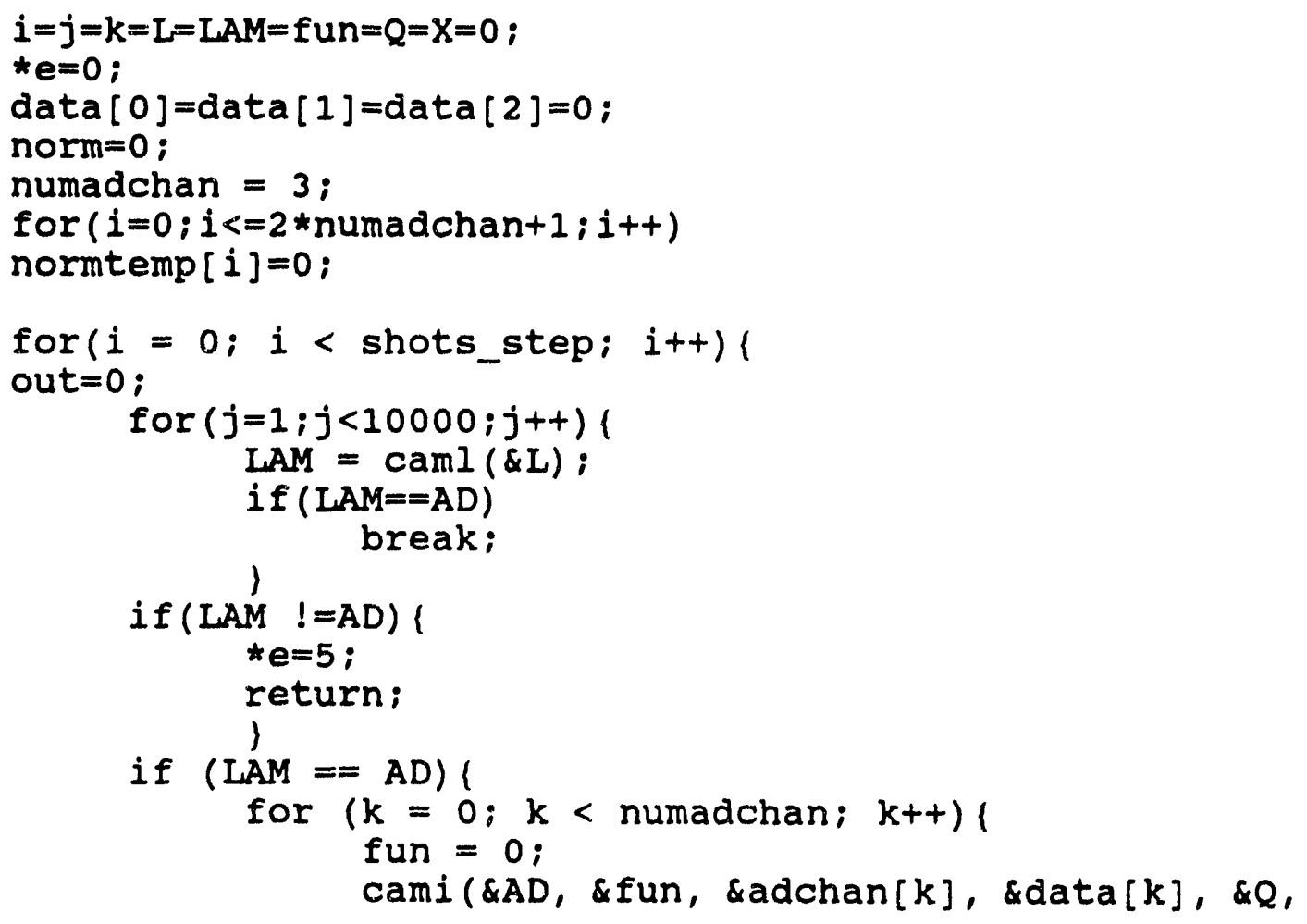




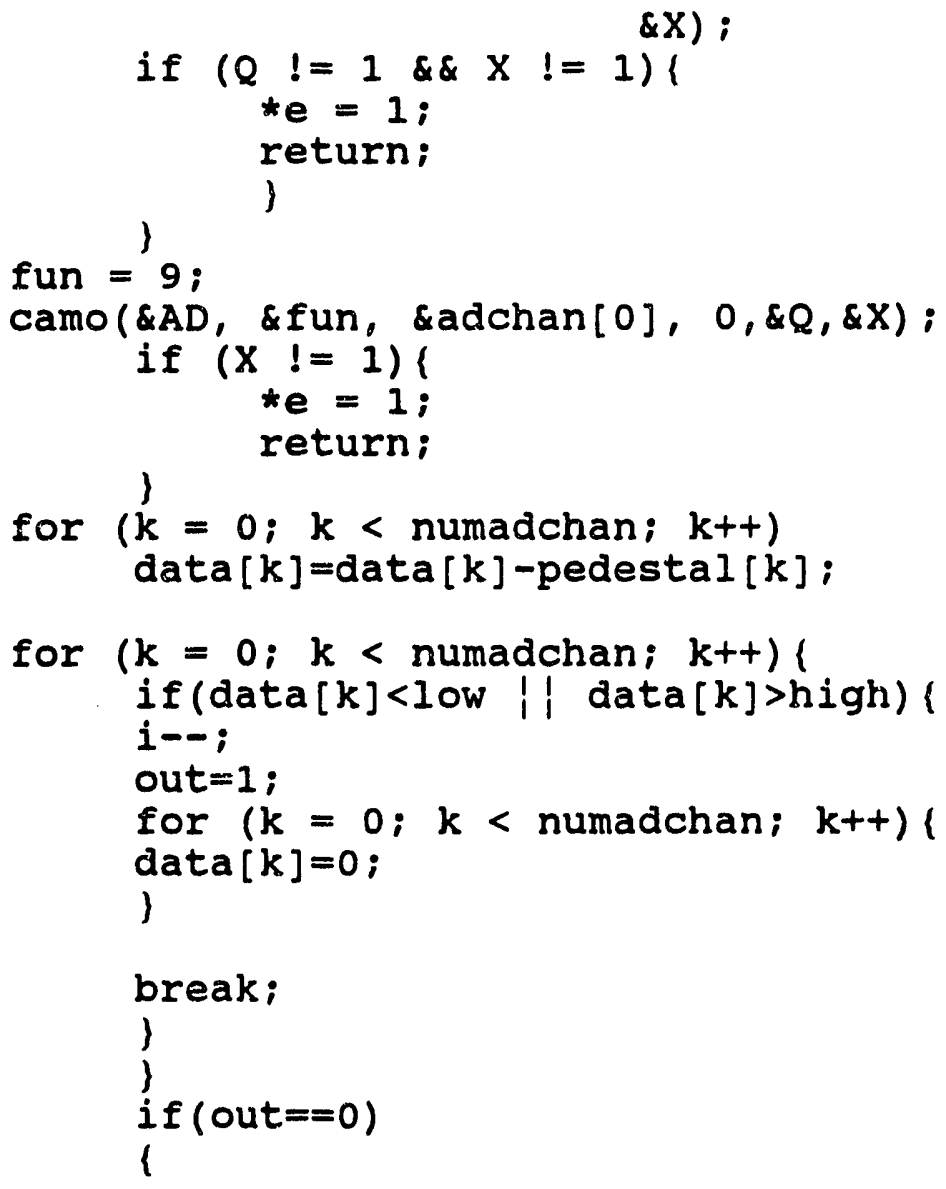




\section{References}

1. A. P. Alivisatos, M. F. Arndt, S. Efrima, D. H. Waldeck, and C. B. Harris, J. Chem. Phys. 86, 6540 (1987).

2. E. S. Peterson and C. B. Harris, J. Chem Phys. 91, 2983 (1989).

3. T. F. Heinz, C. K. Chen, D. Ricard, and Y. R. Shen, Phys. Rev. Lett. 48478 (1982).

4. J. Nahme, T. Kessler, R. Markus, M. Chergui, and $N$. Schwentner, J. of Lumin. $40 \& 41,821$ (1988).

5. N. Bloembergen and P. S. Pershan, Phys Rev. 128, 606 (1962).

6. T. F. Heinz, Ph.D. Thesis: University of California, Berkeley, pp. 71-78, (1982).

7. C. R. Cantor and P. R. Schimmel, Biophysical Chemistry, Part 2 (W. H. Freeman and Company, San Francisco, 1980) pp. 392-398.

8. M. Kasha, H. R. Rawls, and M. Ashraf El-Bayoumi, Pure Appl. Chem. 11, 371 (1965).

9. I. Onsager, J. Am. Chem. Soc. 58, 1486 (1936).

10. N. S. Bayliss, J. Chem. Phys. 18, 292 (1950).

11. E. G. McRae, J. Phys. Chem. 61, 562 (1957).

12. N. J. Tro, D. R. Haynes, A. M. Nishimura, and S. M. George, Chem. Phys. Lett. 159, 599 (1989).

13. J. P. Hermann and J Ducuing, Opt. Commun. 6, 101 (1972).

14. We believe that shen and coworkers omitted the cube on the cosine term in ${ }^{s} \chi^{(2)} 2 \times x^{\prime}$ and as a result they report a value of $34^{\circ}$ for $\beta$ rather than the value that we calculate: $38^{\circ}$.

15. K. Kemnitz, N. Tamai, I. Yamazaki, N. Nakashima, and K. Yoshihara, J. Phys. Chem. 90, 5094 (1986).

16. P. Dilazzaro, P. Mataloni, and F. DeMartini, Chem. Phys. Lett. 114, 103 (1985).

17. J. Frenkel, Phys. Rev. 37, 1276 (1931).

18. G. H. Wannier, Phys. Rev. 52, 191 (1937). 
19. R. S. Knox, Theory of Excitons (Academic Press, New York, 1980).

20. I. Y. Fugol', Adv. Phys. 27, 1 (1978).

21. R. S. Knox, J. Phys. Chem. Solids 9, 265 (1959).

22. J. C. Phillips, Phys. Rev. 136, A 1714 (1964).

23. J. Hermanson, Phys. Rev. 150, 660 (1966).

24. J. Hermanson and J. C. Phillips, Phys. Rev. 150, 652 (1966).

25. D. L. Dexter, Phys. Rev. 101, 48 (1956).

26. A. S. Davydov, Theory of Molecular Excitons, translated by M. Kasha and M. Oppenheimer (McGraw-Hill Book Company Inc., New York, 1962).

27. O. Schnepp and K. Dressler, J. Chem. Phys. 33, 49 (1960).

28. G. Baldini, Phys. Rev. 128, 1562 (1962).

29. J. Jortner, L. Meyer, S. A. Rice and E G. Wilson, J. Chem. Phys. 42, 4250 (1965).

30. S. J. Nettel, Phys. Rev. 212, 425 (1961).

31. D. Beaglehole, Phys. Rev. Lett. 15, 551 (1965).

32. S. A. Rice and J. Jortner, J. Chem. Phys, 44, 4470 (1966).

33. B. Raz and J. Jortner, Proc. Roy. Soc. Lond. A. 317, 113 (1970).

34. B. Raz and J. Jortner, Chem. Phys. Lett. 4, 511 (1970).

35. A. Gedanken, Z. Karsch, B. Raz, and J. Jortner, Chem. Phys. Lett. 20, 163 (1973).

36. I. Messing, B. Raz, and J. Jortner, Chem. Phys. 23, 23 (1977).

37. S. Kubota, A. Nakamoto, T. Takahashi, S. Konno, T. Hamada, M. Mijajima, A. Hitachi, E Shibamura, and T. Doke, Phys. Rev. B 13, 1649 (1976).

38. I. Steinberger, C. A. Alturi, and O. Schnepp, J. Chem. Phys. 52, 2723 (1970) 
39. D. K. Anderson, Phys. Rev. 137, A21 (1965).

40. U. Asaf and I. T. Steinberger, Phys. Lett. 34A, 207 (1971).

41. L. S. Miller, S. Howe, and W. E. Spear, Phys. Rev. 166, 871 (1968).

42. I. T. Steinberger and U. Asaf, Phys. Rev. B 8, 914 (1973).

43. G. L. Pollack, Rev. Mod. Phys. 36748 (1964).

44. F. Colleti and A. M. Bonnot, Chem. Phys. Lett. 55, 92 (1978).

45. J. Bardeen and W. Shockley, Phys Rev. 80, 72 (1950).

46. U. Asaf and I. T. Steinberger, Phys. Rev B. 10, 4464 (1974).

47. P. Laporte and I. T. Steinberger, Phys. Rev. A 15,2538 (1977).

48. P. Laporte, J. L. Subtil, U. Asaf, and I. T. Steinberger, and S. Wind, Phys. Rev. Lett. 45, 2138 (1980).

49. R. W. Harris and G. T. Clayton, Phys. Rev. 153, 229 (1967).

50. J. Wörmer and T. Möller, Z. Phys. D. 20,39 (1991).

51. T. Möller, Z. Phys. D. 20, 1 (1991).

52. S. Kubota, A. Nakamoto, T. Takahashi, T. Hamada, E. Shibamura, M. Miyajima, K. Masuda, and T. Doke, Phys. Rev. B. 17, 2762 (1978).

53. R. Kink, A. Lôhmus, and M. Selg, J. Mol. Struct. 61, 309 (1980).

54. M. Martin, J. Chem. Phys. 54, 3289 (1970).

55. N. G. Basov, E. M. Balashov, O. V. Bogdankevitch, V. A. Danilychev, B. N. Kashmikov, N. P. Lantzov, D. D. Khodkevitch (unpublished).

56. T. L. Gilbert and A. C. Wahl, J. Chem. Phys. 47, 3425 (1976).

57. R. S. Mulliken, J. Chem Phys. 52, 5170 (1970). 
58. T. P. Das, A. N. Jette, and R. S. Knox, Phys. Rev, 134, A1079 (1964).

59. A. N. Jette, T. L. Gilbert, and T. P. Das, Phys, Rev. 184, 884 (1969).

60. R. S. Mulliken, Phys Rev. 136, A962 (1964).

61. S. D. Druger and R. S. Knox, J. Chem. Phys. 50, 3143 (1969).

62. H. Y. Sun and S. A. Rice, J. Chem. Phys. 42, 3826 (1965).

63. R. Kink, A. Lôhmus, and M. Selg, Phys. Stat. Sol. (b) 107, 479 (1981).

64. H. J. Kmiecik and M Schreiber, J. Lumin. 37, 191 (1987).

65. K. Nasu and Y. Toyozawa, J. Phys. Soc. Japan 50, 235 (1981).

66. K. Huang and A. Rhys, Proc. Roy. Soc. (London) A204, 460 (1950).

67. J. W. Keto, R. E. Gleason Jr., T. D. Bonifield, G. K. Walters and F. K. Soley, Chem. Phys. Lett. 42,125 (1976).

68. H. D. Wenck, S. S. Hasnain, M. M. Nikitin, K. Sommer, G. Zimmerer, and D. Haaks, Chem. Phys. Lett. 66, 138 (1979).

69. K. Monahan, V. Rehn, E. Matthias, and E. Poliakoff, J. Chem. Phys. 67, 1784 (1977).

70. S. Kubota, M. Hishida, and J. Raun(Gen), J. Phys. C 11, $2645(1978)$.

71. J. W. Keto, R. E. Gleason and F. K. Soley, J. Chem. Phys. 71, 2676 (1979).

72. C. Achermann, R. Brodmonn, R. Haensel, U. Hahn, $G$. Tolhiehn, and G. Zimmerer, J. Lumin. 12, 315 (1976).

73. J. M. Debever, A. Bonnot, A. M. Bonnot, F. Coletti, and $J$ Hanus, Solid State Commun. 14, 989 (1974).

74. R. Brodmann, G. Tolkiehn, and G. Zimmerer, Phys. Stat. Sol. (b) 73, K99 (1976).

75. U. Hahn, N. Schwentner, and G. Zimmerer, Opt. Commun. 21, 237 (1977). 
76. E. Roick, R. Gaethke, G. Zimmerer, and P. Gürtler, solid State Commun. 47, 333 (1983).

77. E. I. Rashba, in Defects in Insulating crystals, ed. by V. M. Tuchkevich and K. U. Shvarts, Zinatue Publishing House, Riga, and Springer Verlag, Berlin (1981) . p. 255.

78. H. J. Kmiecik, M. Schreiber, T. Kloiber, M. Kruse, and G. zimmerer, J. Lumin. 38, 93 (1987).

79. T. Kloiber, H. J. Kmiecik, M. Kruse, M. Schreiber, and G. Zimmerer, J. Lumin. 40 \&1, 593 (1988).

80. T. Suemoto, Y. Kondo, H. Kanzaki, Solid state Commun. 25, 669 (1978).

81. T. Suemoto and H. Kanzaki, J. Phys. Soc. Japan 46, 1554 (1979).

82. R. T. Williams and M. N. Kabler, Phys. Rev. B9, 1897 (1974).

83. O. Dössel, H. Nahme, R. Haensel, and $\mathrm{N}$ Schwentner, $\mathrm{J}$. Chem. Phys. 79, 665 (1983).

84. M. A. Berg, Ph. D. Thesis: Picosecond Dynamics of Reactions in the Liquid Phase: studies of Iodine Photodissociation and Development of New Laser Techniques, University of California, Berkeley, (1985).

85. A. Harris, Ph. D. Thesis: Picosecond Spectroscopy of Chemical Reactions in Liquids, University of California, Berkeley, (1985).

86. M. A. Berg, A. L. Harris, J. K. Brown, and C. B. Harris, Opt. Lett. 9, 50, (1984).

87. J. A. Beattie, R. J. Barriault, and J. S. Brierley, J. Chem. Phys. 19, 1219 (1951).

88. W. B.. Streett, L. S. Sagan, L. A. K. Staveley, J. Chem. Thermo. 5, 633 (1973).

89. A. W. McCown, M. N. Ediger and J. G. Eden, Phys. Rev. A 29,2611 (1984).

90. D. Haaks, M. Swertz, and J. Schaab, Photophysics and Photochemistry above $6 \mathrm{eV}$, (Elsevier Science Publishers, Amsterdam, 1985).

91. W. Gornik, E. Matthias, and D. Schmidt, J. Phys. B. 15, 3413 (1982). 
92. P. Laporte, N. Damany, and J. L. Subtil, Photophysics and Photochemistry Above $6 \mathrm{eV}$ (Elsevier Science Publishers, Amsterdam, 1985) p. 289.

93. H. Sumi, Solid State Commun. 17, 701 (1975).

94. A. L. Harris, J. K. Brown, and C. B. Harris, Ann. Rev. Phys. Chem. 39, 341 (1988).

95. A. Seilmeier and W. Kaiser, Ultrashort Laser Pulses and Applications, ed. W. Kaiser (Springer-Verlag, Berlin, 1988) chapter 7 .

96. L. Cordis, G. Ganteför, J. Hesslich, and A. Ding, Z. Physik D 3, 323 (1986).

97. V. Saile, Appl. Opt. 234115 (1980).

98. S. Kubota, M. Hishida, M. Suzuki, and J. Ruan, Phys. Rev. B 20,3486 (1979).

99. E. Schuberth and M. Creuzburg, phys. stat. sol. (b) 90 , 189 (1978).

100. D. J. Russell, Ph. D. Thesis: Vibrational Relaxation in Liquids: Comparisons between Gas Phase and Liquid Phase Theories, University of California, Berkeley, p. 206, $(1990)$. 

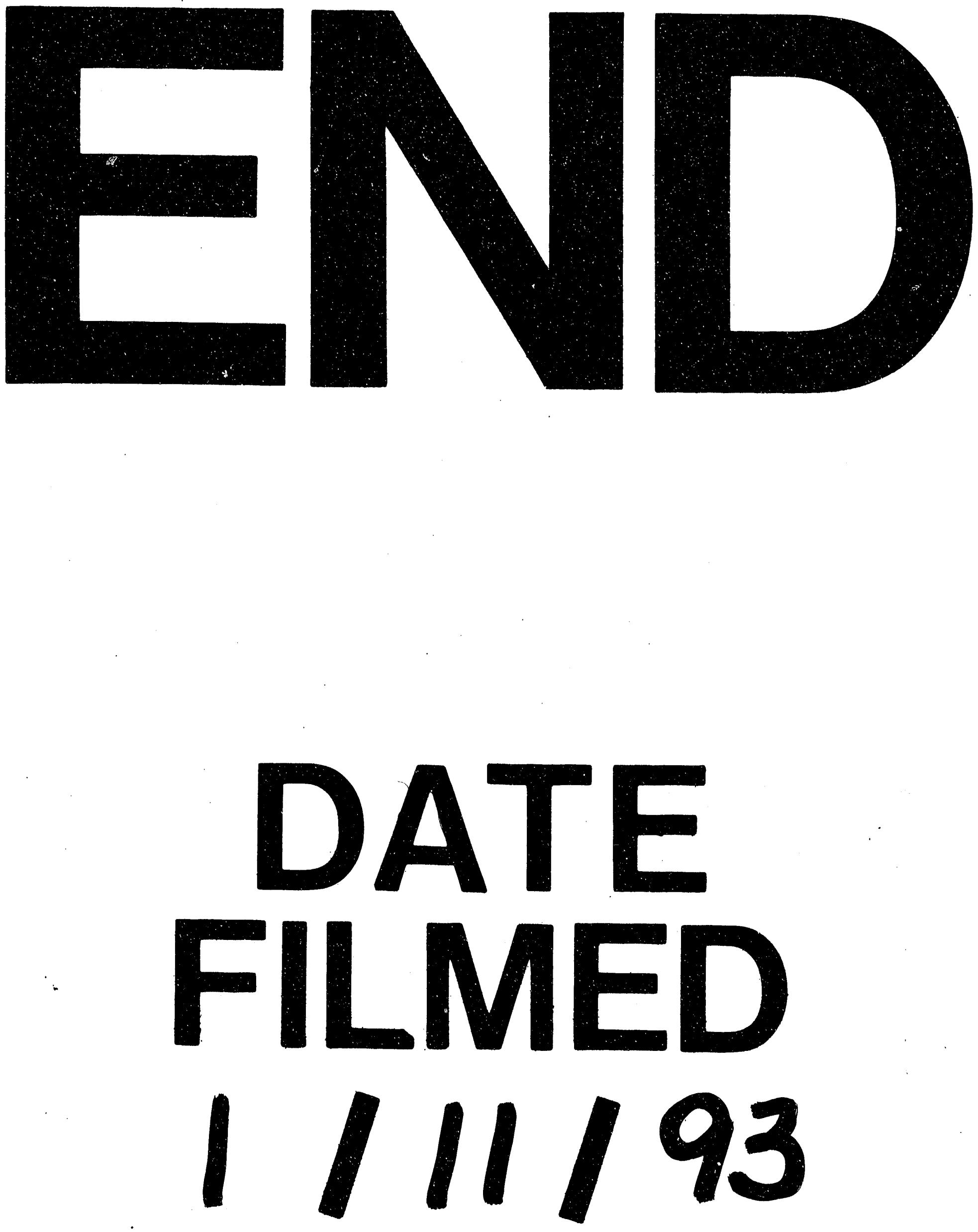
